\title{
Impact of Complex Network Topology on Synchronization Dynamics
}

\section{Dissertation}

zur Erlangung des mathematisch-naturwissenschaftlichen Doktorgrades

"Doctor rerum naturalium"

der Georg-August-Universität Göttingen

vorgelegt von

\section{Carsten Grabow}

aus Uelzen

Göttingen 2012 


\section{Mitglieder des Betreuungsausschusses:}

Prof. Dr. Marc Timme (Betreuer, Referent)

Max-Planck-Institut für Dynamik und Selbstorganisation, Göttingen

Max-Planck-Forschungsgruppe Netzwerk-Dynamik

Prof. Dr. Annette Zippelius (Referentin)

Georg-August-Universität Göttingen

Institut für Theoretische Physik

Prof. Dr. Stephan Herminghaus

Max-Planck-Institut für Dynamik und Selbstorganisation, Göttingen Abteilung Dynamik komplexer Fluide

Tag der mündlichen Prüfung: 27.01.2012 
I hereby declare that this thesis was completed independently and without any unauthorized support. 
Parabase

Freudig war vor vielen Jahren, Eifrig so der Geist bestrebt, $\mathrm{Zu}$ erforschen, zu erfahren, Wie Natur im Schaffen lebt. Und es ist das ewig Eine, Das sich vielfach offenbart; Klein das Große, groß das Kleine, Alles nach der eignen Art.

Immer wechselnd, fest sich haltend, Nah und fern und fern und nah, So gestaltend, umgestaltend Zum Erstaunen bin ich da.

Goethe 
Meinen geliebten Eltern ohne die all dies nicht möglich gewesen wäre 



\section{Contents}

\begin{tabular}{lll}
\hline 1 & Introduction and Overview & 9
\end{tabular}

2 Fundamentals 15

2.1 Basic notions from graph theorv . . . . . . . . . . . . . . . . . . . . 15

2.1.1 Graphs and the adjacency matrix . . . . . . . . . . . . . 15

2.1 .2 Degrees and paths ................. . . 15

2.1.3 Shortest paths, connectivity, betweenness and clustering . . 16

2.1.4 Spectral properties and the graph Laplacian . . . . . . . . . 19

2.2 Network structure and dynamics . . . . . . . . . . . . . . . . . 20

2.2 .1 The small-world topology . . . . . . . . . . . . . 20

2.2 .2 Oscillator dynamics on networks . . . . . . . . . . . . . . . . 22

3 Speed of Complex Network Synchronization 25

3.1 Svnchronization in networks of coupled oscillators . . . . . . . . . 26

3.2 The speed of svnchronization in simulations . . . . . . . . . . . . 27

3.3 Quantifving the small-world regime . . . . . . . . . . . . . . 30

3.4 Analytical predictions for the synchronization time . . . . . . . . 31

3.4 .1 Kuramoto oscillators . . . . . . . . . . . . . . . . . . 31

3.4 .2 Rössler oscillators . . . . . . . . . . . . . . . . . . . . 31

3.4.3 Pulse-coupled oscillators . . . . . . . . . . . . . . . 35

3.5 Dependence on topological randomness and degree . . . . . . . . . . 40

3.5.1 Choice of network ensembles . . . . . . . . . . . . . . . . 40

3.5.2 Monotonicitv: networks with fixed in-degree . . . . . . 41

3.5.3 Non-monotonicity: ensembles with fixed average path length 42

3.5 .4 Generic nonlinear dependence ............. . . 44

3.5.5 Similarity between Kuramoto and pulse-coupled oscillators . 46

3.6 Real-world networks . . . . . . . . . . . . . . . . . . . . . . . 49

3.6 .1 Randomizing real-world networks . . . . . . . . . . . . . . 49

3.6.2 Randomized real-world networks synchronize faster . . . . . 50

3.7 Summary and discussion . . . . . . . . . . . . . . 51

4 Small-World Spectra in Mean Field Theory 53

4.1 A new mean field rewiring . . . . . . . . . . . . . . . . . . 54

4.2 A single formula for the entire small-world spectrum . . . . . . . . . 55

4.3 The ordering of the mean field spectrum . . . . . . . . . . . . . 59

4.4 Extreme eigenvalues . . . . . . . . . . . . . . . . . . . 62

4.4 .1 Approximation for small degrees . . . . . . . . . . . . . 63

4.4 .2 Scaling with network size . . . . . . . . . . . . . . 64 
4.4 .3 The smallest eigenvalue. . . . . . . . . . . . . . 64

4.5 Analvtical predictions for random topologies . . . . . . . . 66

4.5.1 Ensembles of svmmetric and asymmetric random matrices . 67

4.5.2 Undirected random networks . . . . . . . . . . . . . . . . . 68

4.5.3 Directed random networks . . . . . . . . . . . . . . . . 69

4.5.4 Predictions for the scaled graph Laplacians . . . . . . . . . . 69

4.6 The entire spectrum in mean field theory . . . . . . . . . . . . . . 71

4.7 Summary and discussion . . . . . . . . . . . . . . . . . . 71

5 Hubs Orchestrate Synchrony

5.1 Experimental findings . . . . . . . . . . . . . . . . . . . 76

5.2 The scale-free topology . . . . . . . . . . . . . . . . . 76

5.3 Pulse-coupled oscillators revisited . . . . . . . . . . . . . . . . 79

5.4 Biophysically more realistic neuron models . . . . . . . . . . . . . . 82

5.4 .1 Conductance-based leaky integrate-and-fire neurons . . . . . 82

5.4 .2 Choice of parameters . . . . . . . . . . . . . . . . . 83

5.4.3 Mechanisms of GDP generation and suppression . . . . . . . 86

5.4.4 Random network topologv .............. 87

5.4.5 Current-based neurons with $\delta$-shaped post-synaptic currents 89

5.5 Summary and discussion . . . . . . . . . . . . . . . 91

6 Conclusions 93

\begin{tabular}{ll}
\hline Acknowledgements & 97
\end{tabular}

\begin{tabular}{lr}
\hline Curriculum Vitae & 99
\end{tabular}

\begin{tabular}{lr}
\hline Bibliography & 102
\end{tabular}

\begin{tabular}{ll}
\hline Nomenclature & 115
\end{tabular} 


\section{Introduction and Overview}

As a collective effort of connected individuals, our world is structured by human activity in many different ways: we are surrounded by global networks of communication, transportation, trade, social relations and media, all of which are examples for complex networks. Also Nature provides us with an abundance of examples, such as the human brain, or gene regulatory networks. But what are complex networks? In everyday language, 'complex' is used as the opposite of 'simple', i.e. as a synonym of 'complicated'. A system with a large accumulation of interacting elements would certainly qualify to be called complex in this sense, but the concept of complexity can be made more precise than that [BP97, GM94, Zie01]: it is not only the sheer number of individual elements that matters but also the architecture and the strength of the interactions between them which shape the collective dynamics of a complex network. The collective dynamics however is in practice neither predictable from, nor reducible to, its individual elements, making the study of those complex network models that are 'simple enough' to be actually manageable especially interesting (cf. e.g. [Est10]).

Due to the rapid technological advance, the decipherment of enormously complex natural networks is currently in progress. For example, our knowledge about the brain or the human genome is growing continuously. Basically, our desire to understand the mechanisms inherent to such networks suggests an approach comprised of two parts. Firstly, we need to develop models that sensibly represent the basic structures of the networks at both microscopic and macroscopic level. Secondly, we have to derive appropriate rules which govern the dynamic interactions.

The seemingly very different types of networks specified above share very basic similarities, such as abstract patterns or simple organizing principles. Generally, order is an essential property of physical systems describing Nature. All natural complex systems are ordered macroscopically to some degree (cf. also [Whi05]). In some cases, this order is obvious and easily quantified. For instance, snowflakes show a high level of order, a six-fold symmetry [BJL01, but patterns can also be observed in different systems and over very different length scales (cf. e.g. Kur84, NNS97, Bal01]). On the one hand, order can be viewed as a static property of structural organization, while on the other hand, it can also be an important aspect of collective dynamics. We discuss both these aspects successively in the following paragraph.

A classical example of structural order is a crystal where atoms form a perfectly periodic array. Theoretical studies first focussed on these 'perfect' systems consisting of such simple structures. Regular topologies like lattices, all-to-all coupled units or mean field models and the other extreme - totally random networks [Gil59, Bol01] - are thought to be almost completely understood.

A question which dates back to the sixties changed this approach, namely: "what 
is the probability that any two people, selected arbitrarily from a large population, such as that of the United States, will know each other?" [TM69]. A more interesting formulation, however, takes into account that, although two persons may not know each other directly, they may share one or more mutual acquaintances. In this sense, any two people are connected through an acquaintance chain. This concept, known as the 'six degrees of separation', refers to the idea that every person is on average approximately six jumps on the social network away from any other person on Earth.

Inspired by this concept, Watts and Strogatz published a seminal work [WS98] essentially founding the science of complex network theory Est10. They characterized the small-world phenomenon as the combination of a small path length meaning that a pair of nodes is connected by only a few edges on average - and a high clustering coefficient - meaning that two nodes connected to a common node are also connected to each other with a high probability. From description in theory they were also able to show the small-world phenomenon to be a feature of several examined real-world networks.

Finally, they converted this idea into a simple model to artificially create smallworld networks. Here, the crucial point is that this model interpolates between totally regular and totally random topologies. Starting with a ring where units only communicate with their direct neighbors, ring connections between neighbors are cut with a probability $q$ and connected to randomly chosen nodes somewhere else in the ring. Due to this rewiring associated with the creation of shortcuts, the average path length drastically decreases. Simultaneously this architecture still exhibits high local clustering. These two properties were suggested to be particularly supportive of synchronization, the adjustment of the internal dynamics of individual elements due to an interaction [PRK01]. Indeed, several detailed studies support this view by showing that at fixed coupling strength small-world networks tend to synchronize for lower connectivity, i.e. fewer connections in the network, than many other classes of networks [BP02, WS98].

Synchronization is intimately related to dynamical order. Here, individual processes in different parts of a system are well adjusted and the system is capable of exhibiting coordinated performance. The brain is the archetype of such a system where the functioning is based on dynamical order [Buz06]. Information processing and control of body functions in the brain are performed by billions of coupled individual elements called neurons. They interact with each other by sending and receiving patterns of electrical activity. Through the collective dynamics of neurons the brain can efficiently model the processes in the 'real' world. Thus, almost all living beings are intrinsically based on concepts of dynamical order. However, even in simple inert physical systems, coordinated action of individual elements can spontaneously develop. Coupled Josephson junctions [Wie96, FPW06 may generate microwave radiation, whereas coordination in semiconductor lasers [KVM00, WP08] may result in an output power that is much larger than expected.

Moreover, synchronization is one of the most frequently observed collective dynamics in many physical and biological systems [ADK ${ }^{+}$08, PRK01, Str04, Boc08]. Often, synchronization is advantageous and desired, for instance in secure communication [KKK02]. In other circumstances however, it can also be detri- 
mental and undesired. For instance, strong synchronous activity is associated with pathological effects in the brain. It is believed to trigger epileptic seizures [MC01, MPBT04, $\mathrm{NCA}^{+} 04$, LBH09, Mil10] and to initiate the tremor in patients with Parkinson disease. The resting Parkinson tremor appears to be caused by a population of neurons located in the thalamus and the basal ganglia, which fire in a synchronized and intrinsically rhythmic manner. This synchronized firing acts as a pacemaker for activating pre-motor areas and the motor cortex which in turn leads to the tremor with a similar frequency [EK90. Here it is important to understand the synchronization mechanisms in detail and to find possible mechanisms to desynchronize these cells [PHT05].

A broad area of research has emerged which studies the conditions under which the coupled units synchronize and when they do not [Str01, NMLH03, PC98]. The resulting findings suggest important key properties of the topological influence on network synchronizability, i.e. the capability of a network to synchronize at all. However, they do not tell much about the speed of synchronization given that a network synchronizes in principle.

For any real system, it matters a great deal how fast the units synchronize or whether the network interactions fail to coordinate the units' dynamics on time scales relevant to the system's function (or dysfunction), cf. [ZTGW04, ZLPT07, JMT08, ZBH09. The applications range from consensus dynamics of distributed decision-making problems for interacting groups of agents OS05] to questions from neuroscience about the speed of the visual processing or olfactory discrimination could be [UM03, TFM96]. Anyway this question is far from being understood completely and currently under active investigation [TWG04, TGW06, Tim06, $\mathrm{QHS}^{+} 08$, $\mathrm{QHC}^{+} 08$. In particular, it is largely unknown how fast small worlds synchronize which leads us to one of the main question addressed in this thesis: what is the typical time scale for synchronization? This means how fast can network units coordinate their dynamics if they are not directly interconnected but interact on large networks of regular, random or small-world topology?

We address this question in Chapter 3 of this thesis. We present the first systematic study of asymptotic synchronization times for networks with topologies ranging from completely ordered, grid-like, to completely disordered, random, including the intermediate, partially disordered class of topologies, the small worlds. So far it has been studied analytically for fully random networks only [TGW06]. Furthermore, we extend the formalism of master stability functions [PC98] to quantify the collective time scale for synchronizing systems, so far only used to determine whether a system may synchronize or not. We find that the synchronization times strongly and systematically depend on the network topology. In particular, at fixed in-degree, stronger topological randomness induces faster synchronization, whereas at fixed path length, synchronization is slowest for intermediate randomness in the smallworld regime. Randomly rewiring real-world neural, social and transport networks confirms this picture GHGT10, GGT11.

Since the seminal work of Watts and Strogatz about small worlds, many different networked systems, ranging from the internet [BKMR00], power grids [WS98], and airline traffic [ASBS00], to polymers [JSB00], metabolic pathways [WF01] and neural circuits AY92] have been discovered that all share these two basic charac- 
teristics: high clustering and short average path lengths (in other words, the smallworld effect). These topological features of small-world networks underlie collective dynamics such as synchronization, diffusion or relaxation processes [PRK01]. Such processes occur in various fields, ranging from opinion formation in social networks [PLR05] and consensus dynamics of agents [OS05], to synchronization in biological circuits [BCDLR10, MMZ04] and relaxation oscillations in gene regulatory networks McM02, GdBLC03, TYHC03. In particular, the asymptotic dynamics on a small world is characterized by the spectrum of its graph Laplacian CDS80. Laplacian eigenvectors [BL07] have received only sporadic attention although they arise in many research fields, from mathematical biology to combinatorial optimization. In general, the study of graph spectra is extremely useful and can be very profitable. The founders of Google computed the Perron-Frobenius eigenvector of the web graph and became billionaires as consequence [HK03, BH09].

Although the small-world models based on rewiring have received massive attention both theoretically and in applications (as certified by the huge number of references to the original work [WS98]), for most of their features analytical predictions are not known to date, cf. [NMW00]. In particular, the spectrum of smallworld Laplacians has only been studied for several specific cases and numerically [Mon99, JJ01, BP02, MO04, KvM11, but a general derivation of reliable analytic predictions is still missing.

Consequently, an analytical expression for the complete small-world spectrum is derived in Chapter 4. It is based on a two-stage mean field approximation we introduced and results in a single formula that covers the entire spectrum from regular to strongly randomized topologies. In doing so we are able to explain the simultaneous dependencies on network size, average degree of nodes and the randomness $q$ of the order of unity, where standard Random Matrix Theory can be applied. Our results [GGT12] allow for analytical insight into empirically observed features of dynamics on small-world networks from various research fields, including biology, physics, engineering and social science.

Besides the small-world architecture, there is another network structure beyond the two extremes of totally regular and random ones, known as the scale-free topology [AB00, DM01, AB02, Cal07] since it is characterized by a heavy-tailed distribution of degree per node with no characteristic scale. For neural circuits this means, although most neurons display local connectivity, a small number of hub neurons, characterized by long-range connections linking large numbers of cells, can confer synchronicity on the network. Thus, the presence of hub neurons, which act as super-connected nodes, has been postulated as a substrate for widespread neural synchronization. Intriguingly, by stimulating single hub neurons one may remove the synchronous collective oscillations completely. When the stimulation is switched off, the synchronicity, but if a non-hub unit driven in the same way, the oscillation stays almost identically as in the undriven state.

Such synchronous oscillations constitute one of the most dominant collective dynamics of complex networks. They occur not only in circuits of nerve cells (neurons) [BH99, Buz06, OLPT10, but in a large range of systems, ranging from metabolic and gene regulatory networks within cells to food webs of crossfeeding species [MHH98, WM00] or even to oscillations in the global climate system 
[SR94, SvRE98. Thus, understanding the functional role of hubs - not only in neuronal circuits - is a task of paramount importance and has recently attracted widespread attention [Per10, ZLZK10, SHK07, MS08, $\mathrm{ASW}^{+} 06$, SR07]. However, the mechanisms underlying the suppression of global oscillations in a neurobiological system have not yet been understood.

We address this question in Chapter 5. We set up a detailed theoretical framework and numerically investigate different neuronal network models to reveal potential mechanisms that underlie the experimentally discovered phenomena.

Large parts of this thesis revolve around our articles [GHGT10, GGT11, GGT12]. Thereby, Chapter 3 is based on GHGT10, GGT11 whereas Chapter 4 is based on GGT12. Work in Chapter 5 has not yet been published.

The thesis is organized as follows. The introduction given in Chapter 1 is followed by Chapter 2 which provides the fundamentals used throughout. Here we provide basic notions from graph theory and outline the different types of considered dynamics - Kuramoto phase oscillators coupled via phase differences, higher-dimensional periodic Rössler systems coupled diffusively as well as neural circuits with inhibitory delayed pulse-coupling - and the underlying network topology.

In Chapter 3 we set up the theoretical framework used to study the synchronization time. In this context we explain methods to measure the synchronization time numerically and present first simulations illustrating the decay of perturbations to full synchrony. In Section 3.4 we derive methods to predict the synchronization times semi-analytically (eigenvalues still have to be determined via numerical diagonalization). In particular, we are the first who systematically extend the master stability function formalism [PC98] - so far only used to determine whether a system is stable or not - to the prediction of the synchronization time. In Section 3.5 we compare the analytical predictions for the synchronization times and the results obtained by extensive computer simulations for network ensembles with fixed in-degree, with fixed average path length and with fixed betweenness centrality, followed by an analysis of generic network ensembles. Comparing network ensembles with a fixed number of edges, it is shown that those in the small-world regime synchronize faster than regular networks but slower than random networks. This is expected intuitively - the characteristic path length is monotonically decreasing while rewiring - and in accordance with the result for synchronizability [BP02, WS98]. Hence, we fix the average characteristic path length and again investigate the dependence of synchronization time on the network's topology. We find that, for a fixed average characteristic path length, networks in the small-world regime again synchronize slower than random networks, but this time even slower than regular networks: we see a non-monotonic dependence on the topological randomness. We compare network ensembles with fixed topological quantities like the betweenness centrality as well as generic ensembles for Kuramoto, Rössler and pulse-coupled oscillators. Moreover, we make analytical predictions of the synchronization times for periodic Rössler systems and observe remarkable similarities between the synchronization times for the Kuramoto and pulse-coupled oscillators. In Section 3.6 the study of synchronization times for real-world networks, rewiring them towards fully random networks, confirms our theoretical results.

In Chapter 4 we introduce a two-stage mean-field theory which well approximates 
the actual small-world spectra. We derived a single master formula that covers the entire spectrum from regular to strongly randomized topologies. The analytic expression explicates the simultaneous dependencies on network size, average degree and randomness $q$. Numerical diagonalization of Laplacians of directed and undirected networks shows that the analytic prediction well approximates the actual eigenvalues, except for extreme parameter settings, such as $q \rightarrow 1$. In particular, the two largest eigenvalues, the smallest eigenvalue and the bulk spectrum are well predicted.

In Chapter 5 we discuss recent experimental findings on the functional role of neuronal hubs and present approaches to uncover possible mechanisms that may explain the discovered phenomenon.

The thesis is summarized in Chapter 6 including a discussion of future work. 


\section{Fundamentals}

In this chapter we give a basic introduction to graph theory. We are only presenting the notions necessary for the purpose of this thesis. Furthermore, we will focus on directed graphs, but will provide accordant definitions for undirected graphs, which can be considered as special cases of directed graphs. For a more complete introduction see e.g. [New10, Wil96, Bol98]. In addition, we introduce the dynamical oscillator models we will use throughout this thesis as well as their underlying network structure.

\subsection{Basic notions from graph theory}

\subsubsection{Graphs and the adjacency matrix}

A graph is a collection of vertices joined by edges. Later we will also refer to the vertices as nodes and to the edges as links.

Throughout this thesis we represent a graph by the adjacency matrix $A$. It is an $N \times N$ matrix where $N$ equals the number of vertices of the graph with elements $A_{i j}$ such that

$$
A_{i j}= \begin{cases}1 & \text { if there is an directed edge from } j \text { to } i \neq j \\ 0 & \text { otherwise }\end{cases}
$$

This definition includes the direction of an edge: it runs from the second index to the first. Therefore, these graphs are called directed graphs.

Undirected graphs are included if $A_{i j}=A_{j i}$ for all $i, j$. Thus, the adjacency matrix $A$ for undirected graphs is symmetric, whereas it is in general asymmetric for directed graphs. With eq. (2.1.1) we exclude edges that connect vertices to themselves, so-called self-loops. We will not allow multi-edges, more than one edge between the same pair of vertices, either.

\subsubsection{Degrees and paths}

In a directed network each vertex has two degrees. The in-degree $k_{i}^{\text {in }}$ counts the ingoing edges connected to vertex $i$, while the out-degree $k_{i}^{\text {out }}$ counts its outgoing edges. In terms of the adjacency matrix they can be written as

$$
k_{i}^{\text {in }}=\sum_{j=1}^{N} A_{i j}, \quad k_{j}^{\text {out }}=\sum_{i=1}^{N} A_{i j} .
$$

The number of edges $M$ in a directed network is equal to the total number of ingoing tips of edges at all vertices, or equivalently, to the total number of outgoing 
tails of edges at all vertices. Thus,

$$
M=\sum_{i=1}^{N} k_{i}^{\text {in }}=\sum_{j=1}^{N} k_{j}^{\text {out }}=\sum_{i, j}^{N} A_{i j} .
$$

For an undirected graph it simplifies to just one degree denoted by $k_{i}$ which counts the edges connected to vertex $i$ :

$$
k_{i}=\sum_{j=1}^{N} A_{i j}
$$

But each edge in an undirected graph has two ends, thus the number of edges here results in

$$
M=\frac{1}{2} \sum_{i=1}^{N} k_{i}=\frac{1}{2} \sum_{i, j}^{N} A_{i j} .
$$

A path in a network is any sequence of nodes such that every consecutive pair of nodes in the sequence is connected by an edge. But while the contained edges in undirected graphs can be traversed in both directions, the edges in directed networks have to be traversed following the right orientation.

The length of a path is the number of edges traversed along the path. We can easily calculate the number of paths of length $p$ between any pair of vertices: $A_{i j}$ is one if there is an edge from vertex $j$ to vertex $i$ for both directed and undirected graphs. Then the product $A_{i k} A_{k j}$ is one if there is a path of length two between these vertices via $k$. Thus, the total number of paths of length two from vertex $j$ to vertex $i$ can be written as

$$
\sum_{k=1}^{N} A_{i k} A_{k j}=A_{i j}^{2}
$$

from which can be deduced by induction that the number of paths of length $p$ from vertex $j$ to vertex $i$ is given by $A_{i j}^{p}$.

\subsubsection{Shortest paths, connectivity, betweenness and clustering}

A length of special interest is the length of a geodesic path or length of a shortest path $l_{i j}$. This is a path such that there exists no shorter path between two nodes. We can now formally define the length of a shortest path from vertex $j$ to vertex $i$ as

$$
l_{i j}:=\min \left\{r \in \mathbb{N} \mid A_{i j}^{r} \neq 0\right\} .
$$

The shortest path length $l_{i j}$ is symmetric for undirected networks, which is in general not the case for directed ones. Then, the average shortest path length of a graph is given by

$$
L=\frac{1}{N(N-1)} \sum_{\substack{i, j=1 \\ i \neq j}}^{N} l_{i j}
$$




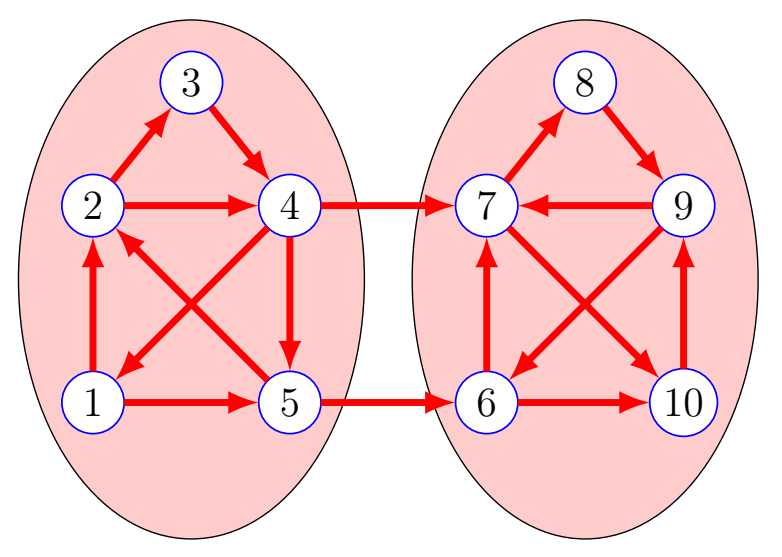

Figure 2.1: Strongly connected components. In this here are two strongly connected subgraphs (shaded areas). However, the whole graph is only weakly connected. For the shaded left subgraph, the sequence $1 \rightarrow$ $2 \rightarrow 3 \rightarrow 4 \rightarrow 5 \rightarrow 2 \rightarrow 4 \rightarrow 1 \rightarrow 5$ is a directed path that solves 'Das Haus vom Nikolaus', a German puzzle for children. In graph theory, such a sequence is known as Eulerian path since every edge in that subgraph is traversed exactly once. Can you find such a path in the right subgraph?

If there is no $r \in \mathbb{N}$ that satisfies eq. (2.1.7), then the vertex $j$ is called disconnected from vertex $i$ and we set $l_{i j}:=\infty$. Otherwise vertex $j$ is called connected to vertex $i$. A directed graph is called strongly connected if every vertex $j$ is connected to every other vertex $i$ in the graph, i.e. $l_{i j}<\infty$ for all $i, j$ in the graph. It is called weakly connected if $l_{i j}<\infty$ or $l_{j i}<\infty$ for all $i, j$ in the graph, i.e. if there exists either a directed path or an inverted directed path between each pair of vertices $i$ and $j$. An undirected graph is simply called connected if each pair of vertices in the graph is connected, i.e. $l_{i j}<\infty$ for all $i, j$ in the graph.

Closely related to the shortest path length is the betweenness centrality [Fre77]. It measures the extent to which a vertex lies on shortest paths between other vertices. The local betweenness centrality of a vertex $i$ is then defined as the number of shortest paths that pass through it as

$$
b_{i}=\sum_{(j, k)} n_{i}^{(j, k)}
$$

where the sum runs over all possible pairs of vertices $(j, k)$ in the graph and

$$
n_{i}^{(j, k)}=\left\{\begin{array}{ll}
1 & \text { if vertex } i \text { lies on the shortest path from vertex } k \text { to vertex } j \\
0 & \text { otherwise }
\end{array} .\right.
$$

Note that we count the shortest paths in either direction seperately, which means that in an undirected graph each path is effectively counted twice. In exchange, we can apply this definition unmodified to directed graphs as well.

We obtain the global betweenness centrality $B$ by averaging the $b_{i}$ over the 


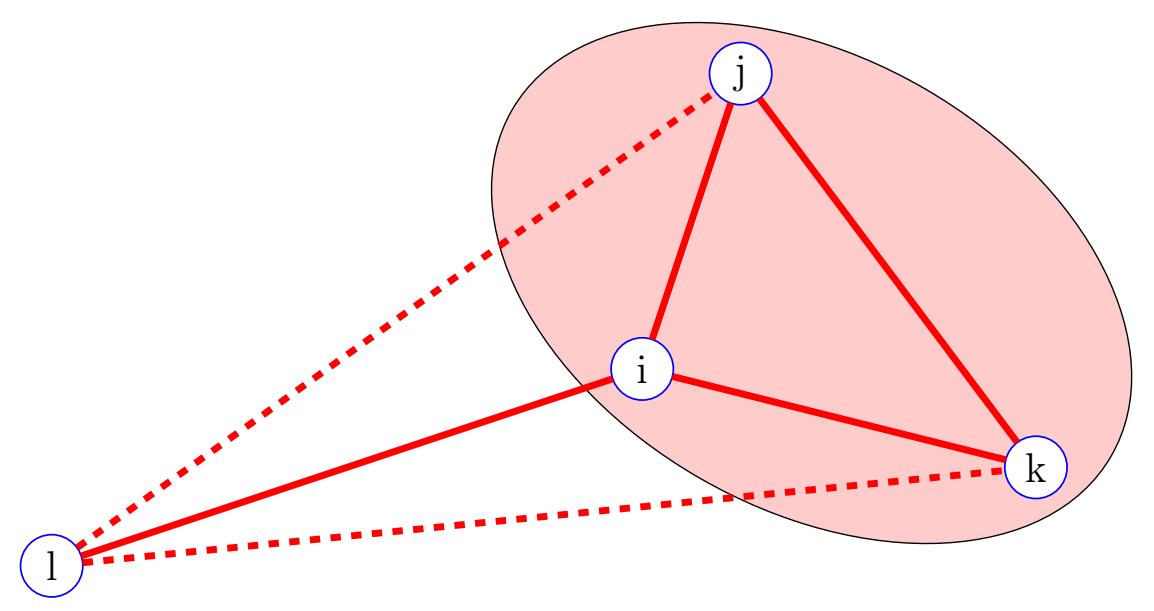

Figure 2.2: Clustering in undirected networks. The clustering coefficient of vertex $i$ is $1 / 3$. Of the possibilities of its neighbours $j, k$ and $l$ to be connected to each other (dashed lines) only one is actually realized (solid line), i.e. one triangle (shaded area) out of three possible triangles is realized.

$N$ vertices, i.e.,

$$
B=\frac{1}{N} \sum_{i=1}^{N} b_{i}
$$

Another important quantity in graph theory is the clustering coefficient. Given that two nodes are connected to a joint third, it measures the likelihood that they are connected to each other as well. Therefore, the local clustering coefficient $c_{i}$ denotes the actual number divided by the possible number of triangles containing a given node $i$ (Fig. 2.2). The local clustering coefficient $c_{i}$ for undirected graphs can be written as

$$
c_{i}=\frac{\frac{1}{2} \sum_{j} \sum_{k} A_{i j} A_{j k} A_{k i}}{\frac{1}{2} k_{i}\left(k_{i}-1\right)}=\frac{A_{i i}^{3}}{k_{i}\left(k_{i}-1\right)},
$$

where $k_{i}$ is the degree (2.1.4) and the sum counts the number of paths of length three which start and end at vertex $i$, i.e. the number of realized triangles (cf. eq. (2.1.6) ).

The generalization to directed networks is not straightforward: In this thesis we use the extension proposed in [Fag07] where we count the number of all possible triangles independent ofã the orientation of their edges (see Fig. 2.3). This number of all possible triangles that contain vertex $i$ is given by

$$
k_{i}^{\mathrm{tot}}\left(k_{i}^{\mathrm{tot}}-1\right)-2 k_{i}^{\mathrm{bi}}
$$

where

$$
k_{i}^{\text {tot }}=k_{i}^{\text {out }}+k_{i}^{\text {in }}
$$



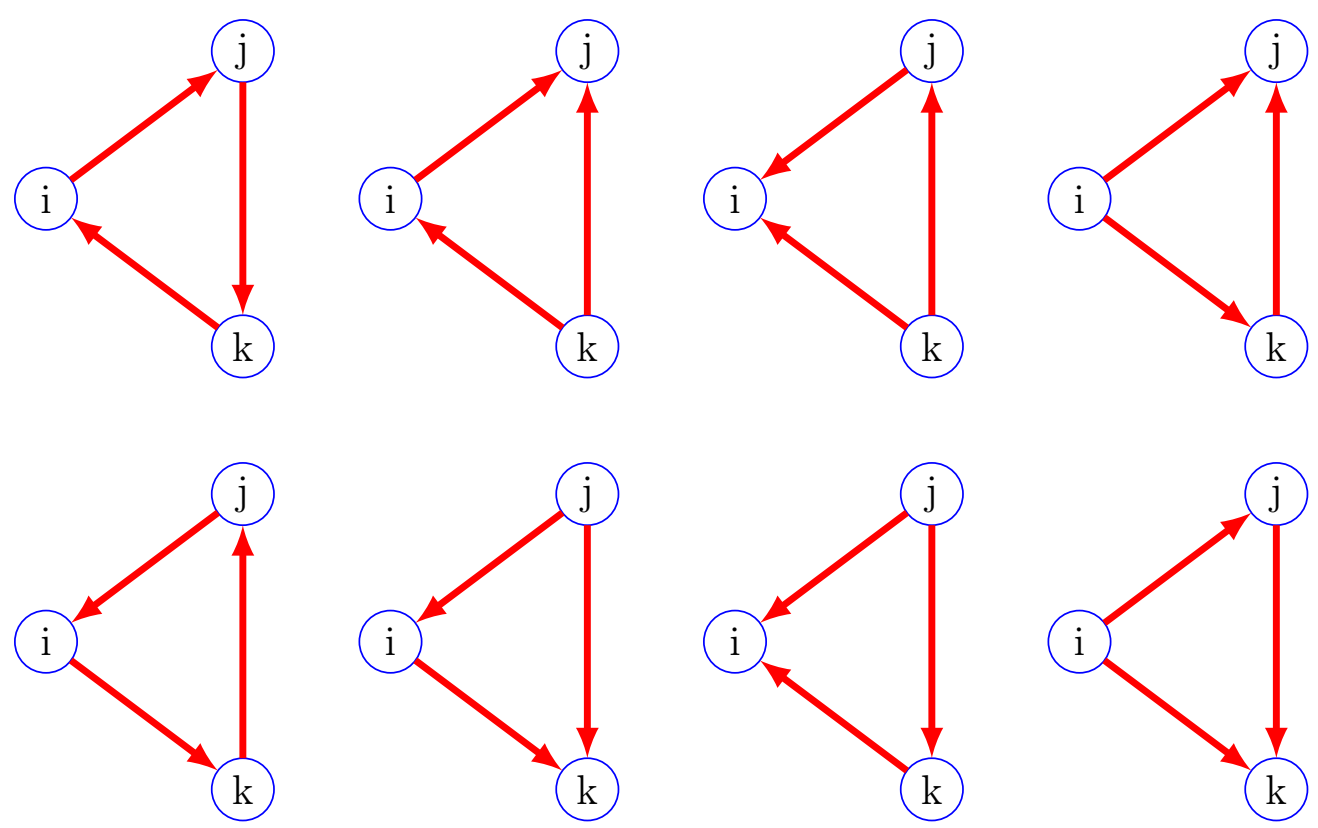

Figure 2.3: Clustering in directed networks. There are eight different triangles containing vertex $i$. The second row can be obtained from the first row by interchanging nodes $j$ and $k$ along with their attached edges.

is the total degree of vertex $i$ and

$$
k_{i}^{\mathrm{bi}}=\sum_{j \neq i} A_{i j} A_{j i}=A_{i i}^{2}
$$

the number of bilateral edges between vertex $i$ and its neighbours, i.e. the number of vertices which are connected to vertex $i$ and simultaneously vertex $i$ is connected to. This leads to

$$
\begin{gathered}
c_{i}=\frac{\frac{1}{2} \sum_{j \neq i} \sum_{k \neq(i, j)}\left(A_{i j}+A_{j i}\right)\left(A_{j k}+A_{k j}\right)\left(A_{k i}+A_{i k}\right)}{k_{i}^{\text {tot }}\left(k_{i}^{\text {tot }}-1\right)-2 k_{i}^{\mathrm{bi}}} \\
=\frac{\frac{1}{2}\left(A+A^{\mathbf{\top}}\right)_{i i}^{3}}{k_{i}^{\text {tot }}\left(k_{i}^{\text {tot }}-1\right)-2 k_{i}^{\text {bi }}}
\end{gathered}
$$

The global clustering coefficient $C$ is then in both cases obtained by averaging the $c_{i}$ over the $N$ vertices, i.e.,

$$
C=\frac{1}{N} \sum_{i=1}^{N} c_{i}
$$

\subsubsection{Spectral properties and the graph Laplacian}

Closely related to the adjacency matrix $A$ which encodes the entire structure of a network is another matrix that can tell us much about the network structure: the graph Laplacian. To clarify its origin let us say a few words on diffusion. It describes 
the spread of particles through random motion from regions of higher concentration to regions of lower concentration. These processes could be also considered on networks such as information spreading in social networks. Therefore suppose some substance on a network of which an amount $\psi_{i}$ is located at node $i$. Then

$$
\frac{d \psi_{i}}{d t}=C \sum_{j=1}^{N} A_{i j}\left(\psi_{j}-\psi_{i}\right)
$$

gives the rate at which $\psi_{i}$ is changing, $C$ being the diffusion constant and $A_{i j}$ the adjacency matrix elements (2.1.1). Rewriting (2.1.19) leads to

$$
\frac{d \psi_{i}}{d t}-C \sum_{j=1}^{N}\left(A_{i j}-k_{i} \delta_{i j}\right) \psi_{j}=0
$$

where $\delta_{i j}$ is the Kronecker delta and $k_{i}$ the degree (2.1.4). Defining

$$
\Lambda_{i j}=A_{i j}-k_{i} \delta_{i j}
$$

we recognize the structure of the ordinary diffusion equation, except that the elements of the Laplacian operator $\nabla^{2}$ are replaced by the matrix elements of $\Lambda$. Hence, $\Lambda$ is called the graph Laplacian, although its importance reaches far beyond diffusion.

Equation (2.1.21) defines the graph Laplacian of undirected networks. Its spectrum is real with the eigenvalues $\lambda_{i}$ ordered as $0=\lambda_{1} \geq \lambda_{2} \geq \ldots \geq \lambda_{N}$. The number of zero eigenvalues of the Laplacian matrix is equal to the number of connected components of the graph.

For directed networks we will use the graph Laplacian defined as

$$
\Lambda_{i j}=A_{i j}-k_{i}^{i n} \delta_{i j}
$$

where the degree $k_{i}$ in undirected networks has been substituted for the in-degree $k_{i}^{i n}(2.1 .2)$.

The eigenvalues $\lambda_{i}$ of directed graphs are complex and ordered as $0=\operatorname{Re} \lambda_{1} \geq$ $\operatorname{Re} \lambda_{2} \geq \ldots \geq \operatorname{Re} \lambda_{N}$. The number of zero eigenvalues of the Laplacian matrix is equal to the number of strongly connected components of the directed graph.

\subsection{Network structure and dynamics}

\subsubsection{The small-world topology}

Consider a graph of $N$ nodes on a one-dimensional ring lattice with periodic boundary conditions. Each node receives directed links from its $k_{i}^{i n} / 2$ nearest neighbors on both sides (for simplicity of presentation we take $k$ and $N$ to be even in this thesis). Furthermore, the in-degree $k_{i}^{i n}=k^{i n}$ for all $i$ is the same for all nodes (see Fig. 2.4). 

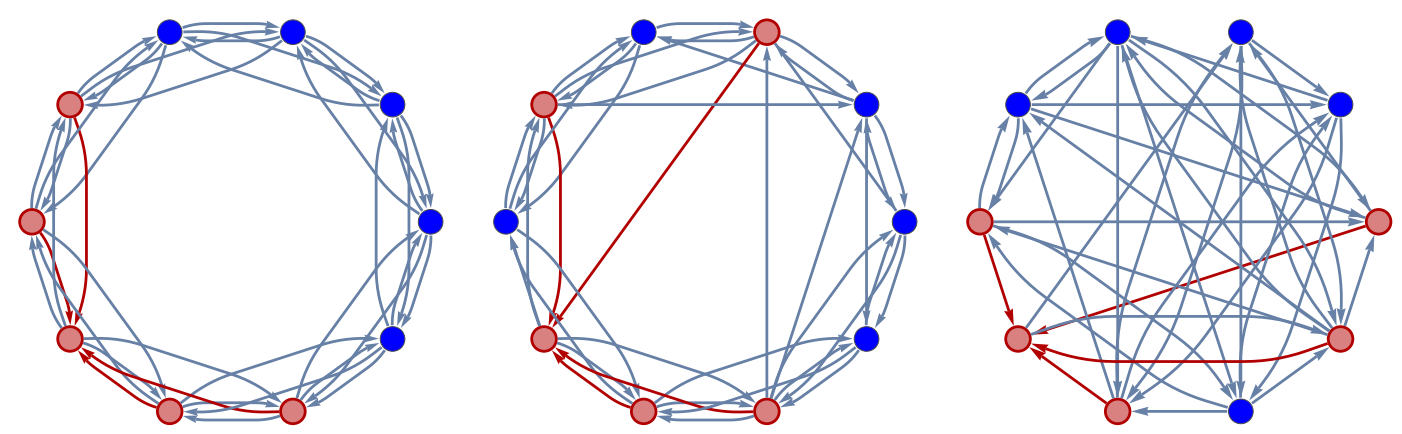

Figure 2.4: Rewiring directed networks. (Cartoon for $N=10, k=4$ ). Single realizations of rewiring for directed networks. From left to right: $q=0$ (regular ring network), $q=0.1$ ('small world') and $q=1$ (random network). While rewiring with probability $q$ the in-degree $k^{i n}$ for each node stays fixed: this can be observed here for a reference node's incoming edges highlighted in red.

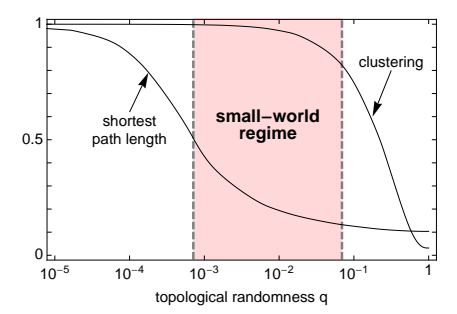

Figure 2.5: Small worlds. In the small-world regime the clustering is high, but the average shortest path length has decreased significantly $(N=1000$, $k^{i n}=20$, averaged over 100 network realizations, clustering coefficient and average shortest path length normalized to one for $q=0$ ).

Randomness is introduced by rewiring where we adapt the standard small-world (SW) model of Watts and Strogatz [WS98 to directed networks [Fag07]. We randomly cut each tail of an outgoing edge with probability $q \in[0,1]$ (also referred to as topological randomness) and rewire it to a node chosen uniformly at random from the whole network (avoiding double edges and self-loops). We do, however, allow the edge to be rewired back to its original position.

An important observation here is that as $q$ varies the in-degree of each node (and with it the average in-degree of the network) is still $k^{i n}$ (see Fig. 2.4). This is due to the fact that we only rewire the tails of outgoing edges.

The directed small-world networks behave as in the original Watts-Strogatz model [WS98 (see Fig. 2.5). Starting with fully regular networks, i.e. the topological randomness $q$ equals zero, the clustering coefficient $\left\langle C\left(q, k^{i n}\right)\right\rangle(($ eq. 2.1.18) $)$ and the average path length $\left\langle L\left(q, k^{i n}\right)\right\rangle$ (eq. 2.1.8) both are large. Here $\langle$.$\rangle denotes$ averaging over network realizations at given $q$ and $k$. On the other side, fully random networks, i.e. for $q=1$, occupy a small clustering coefficient $\left\langle C\left(q, k^{i n}\right)\right\rangle$ and a small average path length $\left\langle L\left(q, k^{i n}\right)\right\rangle$. 
In between, we observe a regime for small randomness $q$ where the clustering coefficient $\left\langle C\left(q, k^{i n}\right)\right\rangle$ is still large in comparison to the fully regular networks, but the average path length $\left\langle L\left(q, k^{i n}\right)\right\rangle$ has already decreased significantly: this regime characterized by these two topological quantities is the small-world regime.

\subsubsection{Oscillator dynamics on networks}

In the following, we briefly introduce the different oscillator models mainly used throughout Chapter 3. Abstractly speaking, oscillators are patterns that return to their original state, in the same orientation and position, after a finite number of generations.

We will investigate three different oscillator types: phase oscillators coupled via phase differences, the Kuramoto oscillators [Kur84, $\mathrm{ABV}^{+} 05$ ], higher-dimensional periodic and chaotic systems coupled diffusively, the Rössler oscillators 2.2 .2 as well as neural circuits with inhibitory delayed pulse-coupling, in the following referred to as pulse-coupled oscillators [MS90].

In each of the three models, the oscillators are coupled via a coupling matrix $J$ which is proportional to the adjacency matrix $A$ defined in eq. (2.1.1).

The entries $J_{i j} \geq 0$ of the coupling matrix consist of a global coupling constant $\sigma$ and are normalized to guarantee that each oscillator $i$ is getting the same input.

The matrix elements $J_{i j}$ are therefore

$$
J_{i j}= \begin{cases}\sigma / k_{i}^{\text {in }} & \text { if } j \text { is connected to } i \neq j \\ 0 & \text { otherwise }\end{cases}
$$

and $J_{i i}=0$ for the diagonal elements.

\section{Kuramoto oscillators}

Consider $N$ Kuramoto oscillators that interact on a directed network. Here, the dynamical variable of each oscillator is $\boldsymbol{x}_{i}:=\theta_{i} \in \mathbb{S}^{1}=2 \pi \mathbb{R} / \mathbb{N}$, i.e. a one-dimensional phase, with its interaction function $\boldsymbol{H}\left(\theta_{i}, \theta_{j}\right):=\sin \left(\theta_{j}-\theta_{i}\right)$. Therefore, the dynamics of phases $\theta_{i}(t)$ of oscillators $i$ with time $t$ satisfy

$$
\frac{d \theta_{i}}{d t}=\omega_{i}+\sum_{j} J_{i j} \sin \left(\theta_{j}-\theta_{i}\right) \quad \text { for } i \in\{1, \ldots, N\}
$$

where $\omega_{i}$ is the natural frequency of oscillator $i$. We consider identical oscillators in this thesis, i.e. $\omega_{i}=\omega$ for all oscillators $i$.

\section{Rössler oscillators}

We also consider a network of Rössler oscillators, both in the chaotic and in the periodic regime. Each elementary Rössler oscillator is described by three variables $\{x(t), y(t), z(t)\}$. The collective dynamics of $N$ coupled, identical Rössler oscillators 
$(i \in\{1,2, \ldots, N\})$ are governed by the equations

$$
\begin{aligned}
\frac{d x_{i}}{d t} & =-y_{i}-z_{i}+\sum_{j=1}^{N} J_{i j}\left(x_{j}-x_{i}\right), \\
\frac{d y_{i}}{d t} & =x_{i}+a y_{i}, \\
\frac{d z_{i}}{d t} & =b+z_{i}\left(x_{i}-c\right)
\end{aligned}
$$

where $a, b$ and $c$ are fixed parameters.

To study the Rössler system in the periodic regime we set the parameters to $a=0.2, b=1.7, c=5.7$. Analogously setting the parameters to $a=0.2, b=0.2$, $c=5.7$ the chaotic regime is gained.

\section{Pulse-coupled oscillators}

Moreover, we investigate the collective dynamics of pulse-coupled (neural) oscillators [JMT08, MS90].

In this case, the dynamical oscillator variables are the membrane potentials $V_{i}(t)$ and delayed discrete output pulses satisfying

$$
\frac{d V_{i}}{d t}=I-\gamma V_{i}+\sum_{j=1 ; j \neq i}^{N} \sum_{m \in \mathbb{Z}} J_{i j} \delta\left(t-\left(t_{j, m}+\Delta\right)\right)
$$

where $I$ is a suprathreshold external current $I>1$ and $\gamma$ the dissipation of the system. Here, each potential $V_{j}$ relaxes towards $I>1$ and is reset to zero whenever it reaches a threshold at unity,

$$
V_{j}\left(t^{-}\right)=1 \Rightarrow V_{j}(t):=0, t_{j, m}:=t \text {, and } m \mapsto m+1 \text {. }
$$

At these times $t_{j, m}$, neuron $j$ sends a pulse that after a delay $\Delta>0$ changes the potential of post-synaptic neurons $i$ in an inhibitory (negative) manner according to (2.2.6) with $\sigma<0$ in (2.2.1).

Equivalently to these ordinary differential equations, there is a simplified approach which represents the state of a one-dimensional oscillator not by its membrane potential but by a phase that encodes the time to the next spike in the absence of any interactions. The state of an individual oscillator $j$ is then represented by a phase-like variable $\phi_{j} \in(-\infty, 1]$ that increases uniformly in time,

$$
d \phi_{j} / d t=1
$$

Upon crossing the firing threshold, $\phi_{j}\left(t^{-}\right)=1$, at time $t$ oscillator $j$ is instantaneously reset to zero, $\phi_{j}(t)=0$, and a pulse is sent. After a delay $\Delta$ this pulse is received by all oscillators $i$ connected to $j$ and induces an instantaneous phase jump given by

$$
\phi_{i}\left((t+\Delta)^{+}\right)=U^{-1}\left(U\left(\phi_{i}(t+\Delta)+J_{i j}\right)\right.
$$


Here, the coupling strengths from $j$ to $i$ are taken to be purely inhibitory $(\sigma<0$ in (2.2.1) $)$ and normalized according to (3.1.3). The rise function $U$, which mediates the interactions, can be derived from (2.2.6) TWG03, and turns out to be monotonically increasing, $U^{\prime}>0$, concave (down), $U^{\prime \prime}<0$, and represents the subthreshold dynamics of individual oscillators. Note that the function $U$ needs to be defined on the entire range of accessible phase values. In particular, inhibitory coupling can lead to negative phase values $\phi_{i}<0$. 


\section{Speed of Complex Network Synchronization}

In this Chapter we present our results related to the first of the main questions raised in the introduction: What is the typical time scale for synchronization, i.e.how fast can oscillators coordinate their dynamics if they are not directly interconnected but interact on large networks of regular, random or small-world topology?

We address this question by computer simulations as well as analytical predictions. All results are derived for the simplest of all regular states, the synchronous periodic state, in which all oscillators exhibit identical dynamics. However, also other settings are imaginable: cluster states in which two or more groups of synchronized oscillators exist [EPG95, EPG98] or systems with inhomogeneities in the dynamical and topological parameters $\left[\mathrm{DTD}^{+} 04\right]$ can be treated similarly. We study the effect of topology on the synchronization time of directed networks which exhibit different dynamics introduced in the previous Chapter: Kuramoto phase oscillators coupled via phase differences (2.2.2), higher-dimensional periodic Rössler systems coupled diffusively (2.2.2) as well as neural circuits with inhibitory delayed pulsecoupling (2.2.2). Synchronization time is a measure of how quickly the network re-synchronizes after being perturbed from a synchronized state.

We present the first systematic study of asymptotic synchronization times for networks with topologies ranging from completely ordered, grid-like, to completely disordered, random, including the intermediate, partially disordered class of topologies, the small worlds. So far it has been studied analytically for fully random networks only [TGW06].

This Chapter is organized as follows. In Section 3.1 we first introduce the concept of synchronization time in networks of coupled oscillators, the central quantity of this Chapter. We then present first simulations including the numerical measurement of synchronization times. In Section 3.4 we derive analytical predictions for the synchronization times. In partiicular, we extend the master stability function formalism PC98 to determine the synchronization speed. In Section 3.5 we compare the analytical predictions for the synchronization times and the results obtained by extensive computer simulations for network ensembles with fixed in-degree, with fixed average path length and with fixed betweenness centrality, followed by an analysis of generic network ensembles. In Section 3.6 the study of synchronization times for real-world networks - rewiring them towards fully random networks - confirms our theoretical results. We close in Section 3.7 with a summary and a discussion of further work. 


\subsection{Synchronization in networks of coupled oscillators}

We assume identical oscillators which leads to identical or complete synchronization. The equation of motion for the uncoupled oscillators is given by

$$
\frac{d \boldsymbol{x}_{i}}{d t}=\boldsymbol{F}\left(\boldsymbol{x}_{i}\right)
$$

where the $m$-dimensional vector $\boldsymbol{x}_{i}=\left\{x_{i, 1}, \ldots, x_{i, m}\right\}$ refers to the components of each oscillator $i \in\{1, \ldots, N\}$ and $\boldsymbol{F}: \mathbb{R}^{m} \mapsto \mathbb{R}^{m}$ defines the dynamics. Although each oscillator evolves in an $m$-dimensional state space, we consider couplings via one coordinate only.

Therefore, we can describe the connection of $N$ oscillators in a directed network by a coupling matrix $J$ that consists of zero and non-zero elements to specify which oscillators are coupled to which other ones. Thus, the coupling matrix $J$ is proportional to the adjacency matrix $A$ defined in eq. (2.1.1).

The matrix elements $J_{i j}$ are given by

$$
J_{i j}=\left\{\begin{array}{ll}
\sigma / k_{i}^{\text {in }} & \text { if } j \text { is connected to } i \neq j \\
0 & \text { otherwise }
\end{array},\right.
$$

where $\sigma$ is a global coupling constant and $1 / k_{i}^{\text {in }}$ a normalization factor that guarantees a homogeneous total input

$$
\sum_{j=1}^{N} J_{i j}=k_{i}^{\text {in }} \frac{\sigma}{k_{i}^{\text {in }}}=\sigma
$$

such that no specific oscillator receives distinguished couplings.

Directly related to the coupling matrix $J$ is the scaled graph Laplacian $\Lambda$ proportional to the one introduced in eq. (2.1.22) in Chapter 2

$$
\Lambda_{i j}=J_{i j}\left(1-\delta_{i j}\right)-\sigma \delta_{i j}
$$

where $\delta_{i j}$ is the Kronecker-delta. Its eigenvalues $\lambda_{i}$ are complex and ordered as $0=\operatorname{Re} \lambda_{1} \geq \operatorname{Re} \lambda_{2} \geq \ldots \geq \operatorname{Re} \lambda_{N}$. The number of zero eigenvalues of the Laplacian matrix is equal to the number of strongly connected components (SCCs) of the network. Thus, if the second-largest eigenvalue $\lambda_{2}$ equals zero, the network is split in more than one SCC. Then, it is impossible for the network to achieve a completely synchronized state, which is only possible for strongly connected subnetworks. We are therefore considering networks with only one SCC throughout this thesis, which means $\operatorname{Re} \lambda_{2}>0$.

We describe the dynamics of the interaction by a function $\boldsymbol{H}$ that is a vector function of dimension $m$ of the dynamical variables of two connected oscillators. We assume that this function $\boldsymbol{H}$ is the same function for each pair of connected oscillators. For example, $\boldsymbol{H}$ for the Rössler oscillators [Roe76] is a $3 \times 3$ matrix that only picks out the $x$-component to couple to the other oscillators. 
The coupled equations of motion become

$$
\frac{d \boldsymbol{x}_{i}}{d t}=\boldsymbol{F}\left(\boldsymbol{x}_{i}\right)+\sum_{j=1}^{N} J_{i j} \boldsymbol{H}\left(\boldsymbol{x}_{i}, \boldsymbol{x}_{j}\right)
$$

where $J_{i j}>0$ acts on each oscillator. Since we want to examine the case of identical synchronization, the equations of motion become the same for all oscillators when the system is synchronized. In the synchronous state all oscillators' variables are equal to the same dynamical variable:

$$
\boldsymbol{x}_{1}(t)=\boldsymbol{x}_{2}(t)=\ldots=\boldsymbol{x}_{N}(t)=\boldsymbol{s}(t),
$$

where $\boldsymbol{s}(t)$ is the subspace defined by the constraints in eq. (3.1.6), the synchronization manifold. We assume $\boldsymbol{H}(\boldsymbol{s}(t), \boldsymbol{s}(t))=0$. Furthermore, we assume stability of this state which means that small arbitrary perturbations to each $\boldsymbol{x}_{j}$ die out in the long time limit.

In addition to these dynamical systems with continuous-time coupling we will investigate the pulse-coupled systems introduced in Section 2.2.2 as well in the following.

\subsection{The speed of synchronization in simulations}

We consider directed regular, small-world and random networks which are characterized by increasing rewiring, the topological randomness $q$. By tuning this parameter we interpolate between regular ring networks $(q=0)$ ), small worlds (low $q \ll 1$ ) and fully random networks $(q=1)$ as has been explained in detail in Section 2.2.1.

To analyze the purely topological impact on the synchronization times, we study the network dynamics in its simplest setting: we consider strongly connected networks with fixed in-degree $k^{i n}$ and homogeneous total input coupling strengths (encoded in the coupling matrix $J$ (2.2.1) ) such that full synchrony is achieved from sufficiently close initial conditions for all coupling strengths $\sigma$ [Tim06].

First simulations for three different kinds of oscillators (see Fig. 3.1) show that synchronization becomes an exponential process after some short transients for all fractions $q \in[0,1]$ of randomness. Thus the distance

$$
d(t)=\max _{i, j} \operatorname{dist}\left(\boldsymbol{x}_{i}(t), \boldsymbol{x}_{j}(t)\right)
$$

from the synchronous state decays as

$$
d(t) \sim \exp (-t / \tau)
$$

in the long time limit, where $\operatorname{dist}\left(\boldsymbol{x}, \boldsymbol{x}^{\prime}\right)$ is a function measuring the distance between the two appropriate phase variables $\boldsymbol{x}$ and $\boldsymbol{x}^{\prime}$, taking into account possible periodic boundary conditions.

The characteristic time scale $\tau$ in (3.2.2) is what we call the synchronization time in the following. However, there exist systems as well where the transient until the exponential decay is not negligible [ZTGW04, ZLPT06, JMT08, ZBH09, TMK10]. 

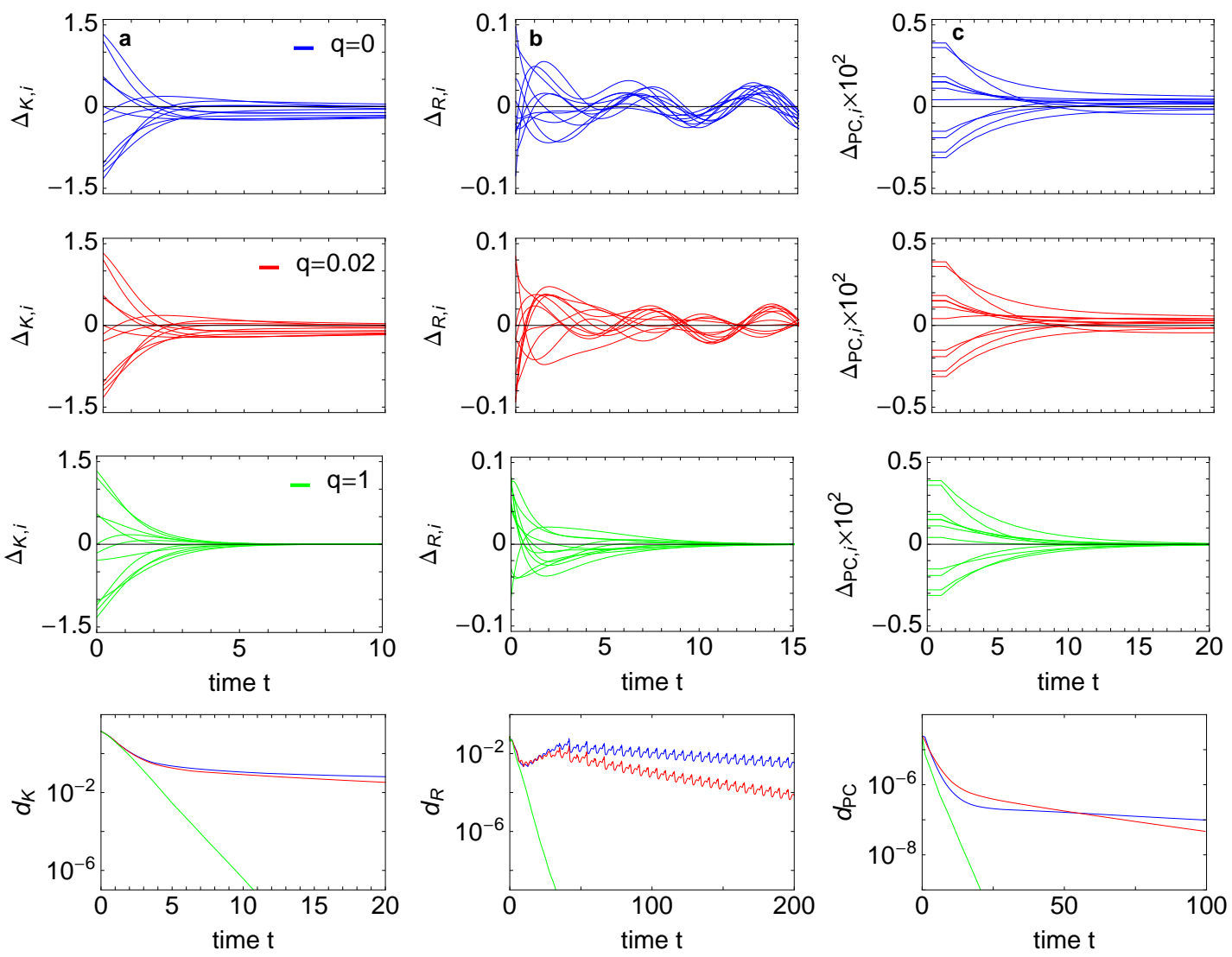

Figure 3.1: Time scales of synchronization of oscillator networks for topological randomness $q \in\{0,0.02,1\}$ (in-degree fixed at $k=20$ ). left column a: Kuramoto oscillators $(\sigma=1)$; b: diffusively coupled periodic Rössler oscillators $(a=0.2, b=1.7, c=5.7, \sigma=2)$; c: pulse-coupled oscillators $(I=1.01, \gamma=1, \sigma=-0.2, \Delta=0.1)$. See equations (3.2.3) - (3.2.5) for the definitions of the variable differences. Plotted in the bottom row are the logarithmized decaying distances (see equations (3.2.2), (3.4.4), (3.4.7) and (3.4.23) $)$.

As one can see in Fig. 3.1 this decay is similar for Kuramoto, Rössler and pulsecoupled oscillators. It depicts the differences of the phase variables (which we have introduced in detail already in Section 3.4) of ten reference oscillators to the corresponding means denoted by [.]:

$$
\begin{aligned}
\Delta_{\mathrm{K}, i}(t) & =\Theta_{i}(t)-\left[\Theta_{j}(t)\right]_{j}, \\
\Delta_{\mathrm{R}, i}(t) & =x_{i}(t)-\left[x_{j}(t)\right]_{j}, \\
\Delta_{\mathrm{PC}, i}(t) & =\tilde{\phi}_{i}(t)-\left[\tilde{\phi}_{j}(t)\right]_{j},
\end{aligned}
$$

with

$$
\tilde{\phi}_{i}(t)= \begin{cases}\phi_{i}(t) & \text { if } \phi_{i}(t) \leq 0.5 \\ \phi_{i}(t)-1 & \text { if } \phi_{i}(t)>0.5\end{cases}
$$


'K' stands for Kuramoto, 'R' for Rössler and 'PC' for pulse-coupled oscillators and these abbreviations will be kept throughout this thesis.

In contrast to the continuous-time dynamics of the Kuramoto and Rössler oscillators, for the pulse-coupled oscillators the phases are measured at discrete 'spiking' times of a reference oscillator. For the 3-dimensional Rössler oscillators only the $x$-coordinates are shown here. The actual dynamical variables for all systems have been introduced in Chapter 2. 


\subsection{Quantifying the small-world regime}

The small-world regime (Section 2.2.1) is characterized by a high global clustering coefficient $C$ (eq. 2.1.18) and a low average shortest path length $L$ (eq. 2.1.8).

To quantitatively fix the small-world regime we take

$$
\frac{\langle L(q, k)\rangle}{L(0, k)}<0.5 \quad \text { and } \quad \frac{\langle C(q, k)\rangle}{C(0, k)}>0.85
$$

throughout this thesis (see Fig. 3.2). However, our results are not sensitive to a change of these values.
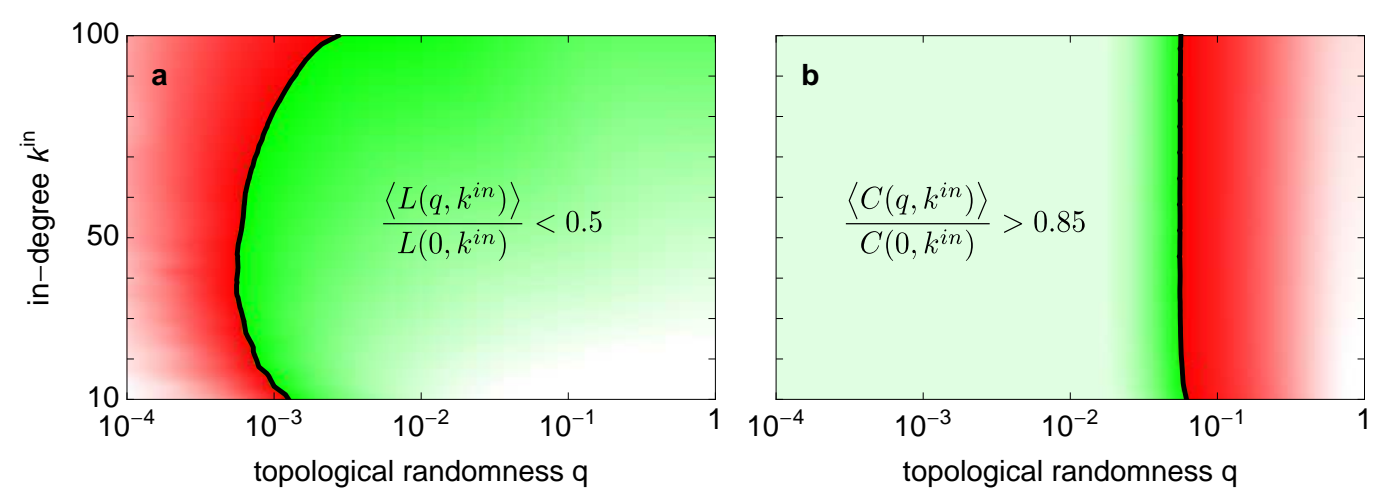

Figure 3.2: Quantifying the small-world regime. The small-world regime is determined by an (a) low shortest path length (green area in a) and a (b) high clustering (green area in b) (see eq. (3.3.1)). The average shortest path length $\langle L(q, k)\rangle$ and the global clustering coefficient $\langle C(q, k)\rangle$ are averaged over 100 network realizations.

As the topological randomness $q$ is changed from zero to one the network continuously interpolates between regular and random topologies. This structural change induces changes in the corresponding graph Laplacian's spectrum and thus has a direct influence on the synchronization speed as is explained in detail in the following Section 3.4. 


\subsection{Analytical predictions for the synchronization time}

In this Section we derive analytical predictions for the synchronization times of the different oscillator types introduced in Section 2.2.2, Additionally, we add remarks on the simulations, the chosen initial conditions and the numerical measurement of the synchronization time.

\subsubsection{Kuramoto oscillators}

The fully synchronous state defined in (3.1.6) here takes the form

$$
\theta_{i}(t) \equiv \theta_{j}(t)=: \theta(t)
$$

As the synchronous periodic orbit analyzed is isolated in state space, the relaxation time continuously changes with possible inhomogeneities, so the qualitative results obtained below are generic and also hold in the presence of small heterogeneities, cf. [DTD $\left.{ }^{+} 04\right]$.

Furthermore, starting from random initial phases in the range $[0, \pi]$ the synchronization dynamics shows a fast transient. After this fast initial evolution all phases are quite similar and the sine function in (2.2.2) can be well approximated by its argument. Linearizing (2.2.2) close to the synchronous state (3.4.1) phase perturbations defined as

$$
\delta_{\mathrm{K}, i}(t):=\theta_{i}(t)-\theta(t)
$$

evolve according to

$$
\frac{d \delta_{\mathrm{K}, i}}{d t}=\sum_{j} \Lambda_{i j} \delta_{\mathrm{K}, j}(t) \quad \text { for } i \in\{1, \ldots, N\} .
$$

Here the stability matrix coincides with the graph Laplacian defined in (3.1.4).

Close to every invariant trajectory the eigenvalue $\lambda_{2}$ of the stability matrix $\Lambda$ that is second largest in real part dominates the asymptotic decay in the long time limit

$$
d_{\mathrm{K}}(t) \sim \exp \left(-t / \tau_{\mathrm{K}}\right)
$$

The distance $d_{\mathrm{K}}(t)$ is given by (3.2.1) where $\operatorname{dist}\left(\theta, \theta^{\prime}\right)$ for Kuramoto oscillators is the circular distance between the two phases $\theta$ and $\theta^{\prime}$ on $\mathbb{S}^{1}$.

$\lambda_{2}$ here determines the asymptotic synchronization time which is given by

$$
\tau_{\mathrm{K}}=-\frac{1}{\operatorname{Re} \lambda_{2}}
$$

\subsubsection{Rössler oscillators}

The evolution of perturbations is characterized by measuring the Euclidean distances

$$
d_{i j}(t)=\sqrt{\left(x_{i}(t)-x_{j}(t)\right)^{2}+\left(y_{i}(t)-y_{j}(t)\right)^{2}+\left(z_{i}(t)-z_{j}(t)\right)^{2}}
$$


between the states of all $N(N-1) / 2$ possible pairs of oscillators $(i, j)$. The asymptotic synchronization time is then determined via the decay of the maximal distance

$$
d_{\mathrm{R}}(t)=\max _{i, j} d_{i j}(t)
$$

\section{The extended master stability function formalism}

A general approach to determine the synchronization time for continuous systems described by (3.1.5) - alternative to the one taken for the Kuramoto oscillators, which does not work for the Rössler oscillators - is to extend the master stability function (MSF) formalism introduced in [PC98] (cf. also [PC90, HCP94b]). Note that this approach does not work for the pulse-coupled oscillators, where the phases are measured at discrete times. But recently, the formalism has been extended to units with time-delayed couplings $\left[\mathrm{KER}^{+} 09\right]$ and to units which are nearly identical [SBN09].

So far this formalism has only been used to determine the stability of networks of coupled oscillators [FJC ${ }^{+} 00$, HCLP09] and nearly all studies have focussed on symmetric undirected networks. More recent studies have also considered directed networks (see e.g. [HCAB05, $\mathrm{CQH}^{+} 10$, Bre10]).

Note that we focus on Rössler oscillators coupled via the $x$-coordinate, but other coupling structures could be treated analogously [Pec98].

Defining infinitesimal perturbations to the synchronous state (3.1.6) in the system described by Eq. (3.1.5) as

$$
\boldsymbol{\delta}_{\mathrm{R}, i}=\boldsymbol{x}_{i}(t)-\boldsymbol{s}(t)
$$

we get the variational equation

$$
\frac{d \boldsymbol{\delta}_{\mathrm{R}, i}}{d t}=\boldsymbol{D} \boldsymbol{F}(\boldsymbol{s}) \boldsymbol{\delta}_{\mathrm{R}, i}-\sum_{j=1}^{N} \Lambda_{i j} \boldsymbol{D H}(\boldsymbol{s}, \boldsymbol{s}) \boldsymbol{\delta}_{\mathrm{R}, i},
$$

where the matrix $\Lambda$ is the graph Laplacian defined in (3.1.4), $\boldsymbol{D F}(\boldsymbol{s})$ and $\boldsymbol{D H}(\boldsymbol{s}, \boldsymbol{s})$ are the Jacobians evaluated along the trajectory $\boldsymbol{s}(t)$.

For the above Rössler system with diffusive coupling via the $x$-coordinate the Jacobian matrices for this block are given by

$$
\boldsymbol{D} \boldsymbol{F}(x, y, z)=\left(\begin{array}{ccc}
0 & -1 & -1 \\
1 & a & 0 \\
z & 0 & x-c
\end{array}\right)
$$

and

$$
\boldsymbol{D} \boldsymbol{H}(x, y, z)=\left(\begin{array}{ccc}
1 & 0 & 0 \\
0 & 0 & 0 \\
0 & 0 & 0
\end{array}\right)
$$

The transformation $\boldsymbol{\delta}^{\prime}{ }_{\mathrm{R}}=O^{-1} \boldsymbol{\delta}_{\mathrm{R}}$, where $O$ is a matrix whose columns are the set of the Laplacian's eigenvectors, diagonalizes the set of equations (3.4.9) and hence leads to a set of decoupled blocks of the form

$$
\frac{d \boldsymbol{\delta}_{\mathrm{R}, i}^{\prime}}{d t}=\left[\boldsymbol{D} \boldsymbol{F}(\boldsymbol{s})-\lambda_{i} \boldsymbol{D} \boldsymbol{H}(\boldsymbol{s}, \boldsymbol{s})\right] \boldsymbol{\delta}_{\mathrm{R}, i}^{\prime}
$$



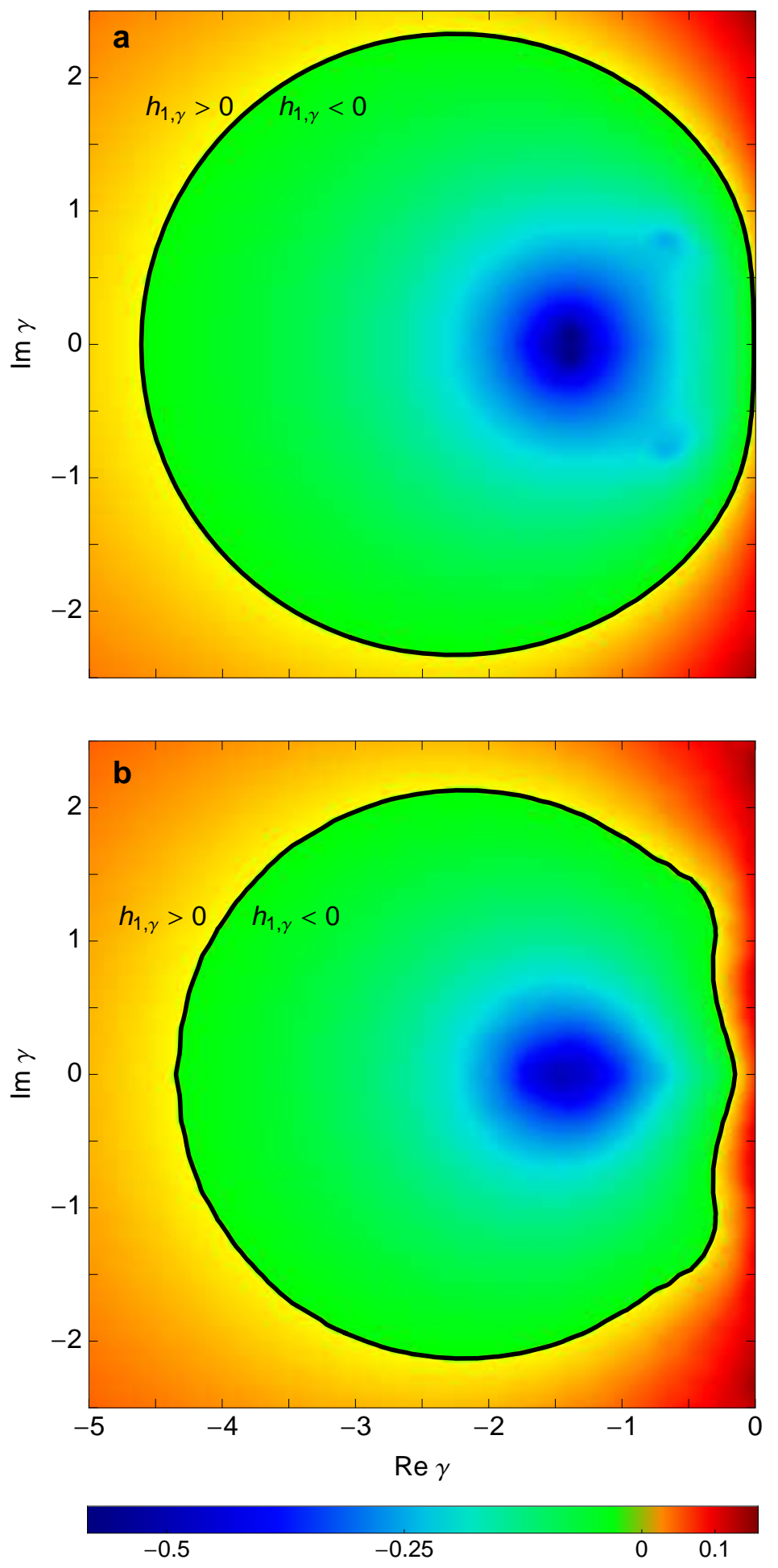

Figure 3.3: Master stability functions $h_{1, \gamma}$ (3.4.14) for the Rössler oscillators. a: periodic regime (parameters set to $a=0.2, b=1.7, c=5.7$ ). b: chaotic regime (parameters set to $a=0.2, b=0.2, c=5.7$ ).

The black contours show the MSF equal to zero, i.e. separate the stable from the unstable regions. 


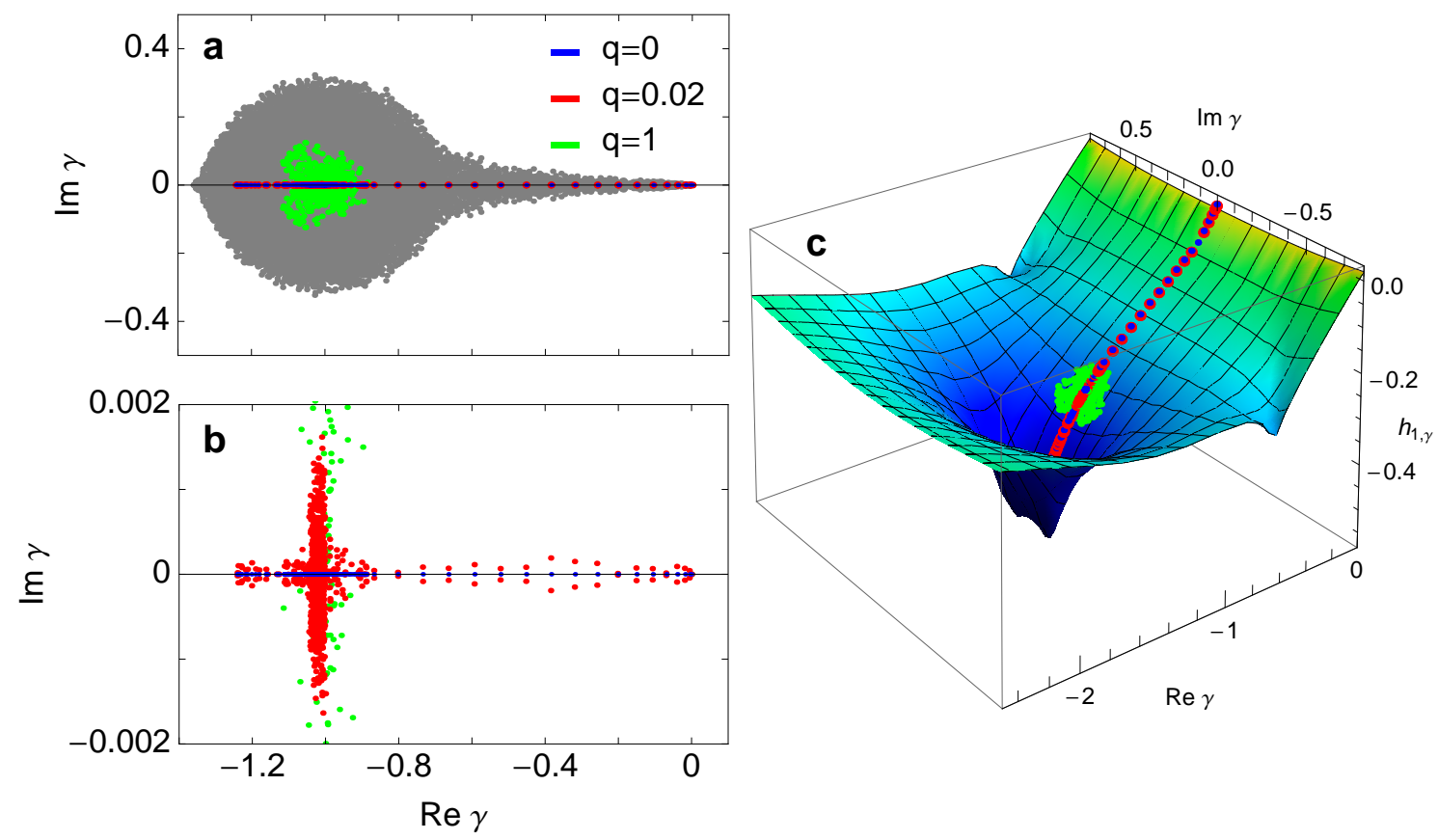

Figure 3.4: The eigenvalue distribution of the graph Laplacian directly links to the system's stability and synchronization dynamics. a: in gray the whole complex spectrum of the graph Laplacians in the ranges $q \in[0,1]$ and $k \in[10,100]$, blue: eigenvalues for $q=0, k=50$ (purely real due to the initial ring symmetry), red: eigenvalues in the SW regime with $q=0.02, k=50$, green: eigenvalues for $q=1, k=50$. b: zoom to the real axis, eigenvalues for $k=50$ and $q \in\{0,0.02,1\}$ with colors as above. c: Evaluating the master stability function $h_{1, \gamma}$ : each eigenvalue $\lambda_{i}$ (for $i \in\{2, \ldots, N\}$ ) of a network realization (points and colors as in $\mathbf{a}$ and $\mathbf{b}$ ) leads to a largest Lyapunov exponent $h_{1, i}$ (3.4.14) - if all these are negative the system synchronizes and the maximal exponent $h_{1, \max }$ determines the asymptotic synchronization time (3.4.15).

with the $\lambda_{i}$ being the eigenvalues of the Laplacian matrix $\Lambda$. The above-given Jacobians evaluated in the synchronized state $\boldsymbol{s}(t)$ are the same for each block, hence the blocks only differ by the scalar multiplier $\lambda_{i}$.

Thus these blocks could be evaluated all at once by setting

$$
\frac{d \boldsymbol{\delta}_{\mathrm{R}, i}^{\prime}}{d t}=[\boldsymbol{D F}(\boldsymbol{s})-\gamma \boldsymbol{D} \boldsymbol{H}(\boldsymbol{s}, \boldsymbol{s})] \boldsymbol{\delta}_{\mathrm{R}, i}
$$

in dependence on the complex coupling parameter $\gamma=\alpha+\mathrm{i} \beta$. The imaginary part $\beta$ may be interpreted as a 'rotation' taking place between the several decaying eigenmodes of the system $\left[\mathrm{PCJ}^{+} 97\right]$.

The system actually synchronizes if

$$
h_{1, i}=\lim _{t \rightarrow \infty} \frac{1}{t} \log \frac{\left|\boldsymbol{\delta}_{\mathrm{R}, i}^{\prime}(t)\right|}{\left|\boldsymbol{\delta}_{\mathrm{R}, i}^{\prime}(0)\right|}<0
$$


for all $i \in\{2, \ldots, N\}$. Here $h_{1, i}$ is the largest Lyapunov exponent corresponding to the mode of eigenvalue $\lambda_{i}$ (see e.g. Ott93).

The largest Lyapunov exponent $h_{1, \gamma}$ in dependence on the complex coupling parameter $\gamma$ is also called the master stability function and plotted in Fig. 3.3 for the periodic and chaotic Rössler oscillators. We calculate the largest Lyapunov exponent following the numerical procedure described in [HCLP09]. It is evident that the $\lambda_{1}=0$-mode is parallel to the synchronization manifold while all the other modes are transverse to it.

To obtain the asymptotic synchronization time we extract the largest Lyapunov exponent $h_{1, i}$ with the minimal absolute value out of the $N-1$ maximal exponents, namely

$$
h_{1, \max }=\max _{i \geq 2} h_{1, i}
$$

The synchronization time for the Rössler oscillators is then given by

$$
\tau_{\mathrm{R}}=-\frac{1}{h_{1, \max }}
$$

$h_{1, \max }$ dominates the decay towards the synchronized state, but note the nonlinear dependence on the eigenvalues of the Laplacian matrix (3.4.12). Only for the simple 1-dimensional Kuramoto oscillators there is a direct relation, since here there is linear and unbounded coupling, i.e. the larger the global coupling the faster the synchronization speed.

In order to find a value for the global coupling parameter $\sigma$ - encoded in $\Lambda$ according to (3.1.4) - that leads to synchronization, one calculates the whole spectrum of possible eigenvalues (see Fig. 3.4) to guarantee that each one is located in the stable region, i.e. in the region where the MSF takes only negative values. Note that the MSF for uncoupled periodic Rössler oscillators is zero, while the MSF for uncoupled chaotic Rössler oscillators is positive. This means that the minimal global coupling constant needed to achieve synchronization is always larger for the chaotic Rössler oscillators than for the periodic ones.

In particular, this means that there is an upper limit on the size of a stable, synchronous array of chaotic Rössler oscillators [HCP94a, HPC95].

\subsubsection{Pulse-coupled oscillators}

The synchronous state $\boldsymbol{s}(t)$ defined in (3.1.6) here takes the form

$$
\phi_{i}(t)=\phi_{0}(t)
$$

for all $i$, which is a self-consistent solution assuming that all neuronal oscillators fire at the same time. Here all oscillators display identical phases $\phi_{0}(t)$ on a periodic orbit such that $\phi_{0}(t+T)=\phi_{0}(t)$ with the period

$$
T=\Delta+1-\alpha
$$

where

$$
\alpha=U^{-1}(U(\Delta)+\sigma)
$$


Note that here in contrast to the Kuramoto oscillators the period is different from the one of a free oscillator TWG02].

A perturbation

$$
\boldsymbol{\delta}_{\mathrm{PC}}(0)=: \boldsymbol{\delta}_{\mathrm{PC}}=\left(\delta_{\mathrm{PC}, 1}, \ldots, \delta_{\mathrm{PC}, N}\right)
$$

to the phases is defined as

$$
\delta_{\mathrm{PC}, \mathrm{i}}=\phi_{i}(0)-\phi_{0}(0)
$$

The initial condition for the phases of the pulse-coupled oscillators is a random perturbation $\boldsymbol{\delta}_{\mathrm{PC}}$ from the globally synchronized state $\boldsymbol{\delta}_{\mathrm{PC}}=\mathbf{0}$. The perturbation components $\delta_{\mathrm{PC}, i}$ are each drawn independently from a uniform distribution on $[-\delta, \delta]$. The condition $\delta<\frac{\Delta}{2}$ derived in [TGW06] (recall that $\Delta$ is the delay time) ensures that the globally synchronized state is stable. This guarantees that all pulse-coupled oscillators reach the threshold before any pulses are received.

A sufficiently small perturbation $\boldsymbol{\delta}_{\mathrm{PC}}$ asymptotically converges exponentially with time to a constant vector. Subtracting the asymptotic phase shift,

$$
\boldsymbol{\delta}_{\mathrm{PC}}^{\prime}(t):=\boldsymbol{\delta}_{\mathrm{PC}}(t)-\lim _{s \rightarrow \infty} \boldsymbol{\delta}_{\mathrm{PC}}(s),
$$

the distance

$$
d_{\mathrm{PC}}(n T):=\max _{i}\left|\delta_{\mathrm{PC}, i}^{\prime}(n T)\right|
$$

from the synchronous state $\left(\delta_{\mathrm{PC}, i}^{\prime} \equiv 0\right)$ decays as

$$
d_{\mathrm{PC}}(n T) \sim \exp \left(-\frac{n T}{\tau_{\mathrm{PC}}}\right)
$$

as $n \rightarrow \infty$, defining a synchronization time $\tau_{\mathrm{PC}}$.

To understand how the speed of synchronization depends on the dynamical and network parameters, we analyze how perturbations $\boldsymbol{\delta}_{\mathrm{PC}}$ to the synchronous state evolve in time. Following [TWG02] we first define a nonlinear stroboscopic map

$$
\boldsymbol{\delta}_{\mathrm{PC}}(n T)=\tilde{G}\left(\boldsymbol{\delta}_{\mathrm{PC}}((n-1) T)\right)
$$

for the perturbations. Note that $\boldsymbol{\delta}_{\mathrm{PC}}(T)=\boldsymbol{\delta}_{\mathrm{PC}}(0)$ since no spikes are received before all the oscillators reach the phase threshold for the first time. Hence we first apply the map $\tilde{G}$ in the first period when spikes are received i.e. for $n \geq 2$.

Considering the first order approximation of this period- $T$ map one gets a linear iterative map $G$ given by

$$
\delta_{\mathrm{PC}, i}(n T)=\sum_{j=1}^{N} G_{i j} \delta_{\mathrm{PC}, j}((n-1) T), \quad n \geq 2,
$$

for the perturbations $\delta_{\mathrm{PC}, i}(n T)$ of spike times close to the synchronous orbit of period

$$
T=\ln (I /(I-1))
$$


The matrix elements $G_{i j}$ are defined as

$$
G_{i j}= \begin{cases}p_{i, m}-p_{i, m-1} & \text { if } j \text { is connected to } i \neq j \\ p_{i, 0} & \text { if } j=i \\ 0 & \text { otherwise }\end{cases}
$$

where the variables $p_{i, m}\left(m \in\left\{1, \ldots, k_{i}\right\}\right)$ encode phase jumps evoked by all pulses up to the $m$ th one received [TWG02]. Since the matrix elements (3.4.28) are differences of these $p_{i, m}$, matrix elements $G_{i j}$ and $G_{i j^{\prime}}$ with $j \neq j^{\prime}$ have in general different values depending on the order of incoming signals.

This multi-operator problem [TW08] is induced by the structure of the network together with the pulsed interactions, in particular, by the order of the components of $\boldsymbol{\delta}(0)$. For networks with homogeneous, global coupling different matrices $G$ can be identified by an appropriate permutation of the oscillator indices. But in general this is impossible. However, here we focus on the leaky integrate-and-fire (LIF) dynamics where the matrix $G$ becomes independent of the rank order of the perturbations [TGW06]. Here $U$ takes the form

$$
U(\phi):=\frac{I}{\gamma}\left(1-e^{-\gamma \phi}\right) .
$$

In order to obtain the matrix elements $G_{i j}$ we first calculate

$$
U^{\prime}(\phi)=I e^{-\gamma \phi}
$$

and

$$
U^{-1}(y)=\frac{1}{\gamma} \ln \left(1-\frac{y \gamma}{I}\right)^{-1}
$$

Furthermore we calculate

$$
U^{-1}\left(U(\Delta)+J_{i j}\right)=\frac{1}{\gamma} \ln \left(e^{-\gamma \Delta}-\frac{\gamma}{I} J_{i j}\right)^{-1}
$$

and

$$
U^{\prime}\left(U^{-1}\left(U(\Delta)+J_{i j}\right)\right)=I e^{-\gamma \Delta}-\gamma J_{i j}
$$

This leads to

$$
\begin{aligned}
p_{i, m} & :=\frac{U^{\prime}\left(U^{-1}\left(U(\Delta)+\sum_{l=1}^{m} J_{i j_{l}}\right)\right)}{U^{\prime}\left(U^{-1}\left(U(\Delta)+J_{i j}\right)\right)} \\
& =\frac{I e^{-\gamma \Delta}-\gamma \sum_{l=1}^{m} J_{i j_{l}}}{I e^{-\gamma \Delta}-\gamma J_{i j}}
\end{aligned}
$$

where the sum $\sum_{l=1}^{m} J_{i j_{l}}$ with $m \in\left\{1, \ldots, k_{i}\right\}$ counts up to the $m$ th signal received by neuron $i$ during the considered period.

For homogeneous inhibitory coupling, $\sigma / k<0$ for each existing connection, the elements of the stability matrix are given by

$$
G_{i j}= \begin{cases}g_{0} / k & \text { if } j \text { is connected to } i \neq j \\ 1-g_{0} & \text { if } j=i \\ 0 & \text { otherwise }\end{cases}
$$


with

$$
g_{0}=\frac{\gamma \sigma}{I e^{-\gamma \Delta}+\gamma \sigma}
$$

We let $\mathbf{v}_{i}$ for $i=1,2, \ldots, N$ be the eigenvectors of $G$ with corresponding eigenvalues $\left|g_{1}\right|>\left|g_{2}\right| \geq \ldots \geq\left|g_{N}\right| . G$ is a stochastic matrix and all diagonal entries satisfy $G_{i i}>0$. Hence, the matrix is aperiodic which implies that the eigenvalue $g_{1}=1$ is the largest and is unique, which can be shown with the Geršgorin circle theorem (see e.g. [Var05]) and the Perron-Frobenius theorem [HC99, BL07]. The eigenvector corresponding to the eigenvalue $g_{1}=1$ is $\mathbf{v}_{1}=(1,1, \ldots, 1)^{\top}$ since the row-sums of $G$ are equal to one. Recall that this means the distance vector $d_{\mathrm{PC}}(n)$ does not tend to zero as $n \rightarrow \infty$, but instead to a uniform phase shift (3.4.22)

$$
\lim _{s \rightarrow \infty} \boldsymbol{\delta}_{\mathrm{PC}}(s)=: \boldsymbol{\delta}_{\infty}
$$

which has all components equal, $\left(\boldsymbol{\delta}_{\infty}\right)_{i}=\delta_{\infty}$ for all $i$ (i.e. all the neurons are at the same phase and hence in a globally synchronized state). Furthermore, recall that the distance from the globally synchronized state is given by

$$
d_{\mathrm{PC}}(n T):=\max _{i}\left|\delta_{\mathrm{PC}, i}^{\prime}(n T)\right|
$$

as defined in (3.4.23). Using the fact that $g_{1}=1, \mathbf{v}_{1}=(1,1, \ldots, 1)^{\top}$ and rewriting $\boldsymbol{\delta}_{\mathrm{PC}}$ as a linear combination of the basis of eigenvectors gives

$$
\begin{aligned}
\boldsymbol{\delta}_{\mathrm{PC}}^{\prime}(n T) & =\boldsymbol{\delta}_{\mathrm{PC}}(n T)-\boldsymbol{\delta}_{\infty} \\
& =G^{n} \boldsymbol{\delta}_{\mathrm{PC}}(T)-\boldsymbol{\delta}_{\infty} \\
& =G^{n}\left(\sum_{i=1}^{N} \beta_{i} \mathbf{v}_{i}\right)-\delta_{\infty} \mathbf{v}_{1} \\
& =\left(\beta_{1}-\delta_{\infty}\right) \mathbf{v}_{1}+\sum_{i=2}^{N} \beta_{i} g_{i}^{n} \mathbf{v}_{i}
\end{aligned}
$$

Since $\left(\boldsymbol{\delta}_{\mathrm{PC}}(n T)-\boldsymbol{\delta}_{\infty}\right) \rightarrow \mathbf{0}$ as $n \rightarrow \infty$ and $\left|g_{i}\right|<1$ for $i \geq 2$ it follows from (3.4.42) that

$$
\lim _{n \rightarrow \infty}\left[\left(\beta_{1}-\delta_{\infty}\right) \mathbf{v}_{1}+\sum_{i=2}^{N} \beta_{i} g_{i}^{n} \mathbf{v}_{i}\right]=\left(\beta_{1}-\delta_{\infty}\right) \mathbf{v}_{1}=\mathbf{0}
$$

and hence

$$
\beta_{1}=\delta_{\infty}
$$

This leaves us with

$$
\boldsymbol{\delta}_{\mathrm{PC}}^{\prime}(n T)=\sum_{i=2}^{N} \beta_{i} g_{i}^{n} \mathbf{v}_{i}
$$


Then, since $g_{2}$ is the second largest eigenvalue, taking the infinity norm in (3.4.42) gives

$$
\begin{aligned}
d_{\mathrm{PC}}(n T) & =\max _{j}\left|\left(\sum_{i=2}^{N} \beta_{i} g_{i}^{n} \mathbf{v}_{i}\right)_{j}\right| \\
& =\left|\beta_{2} g_{2}^{n}\right| \max _{j}\left|\left(\mathbf{v}_{2}+\sum_{i=3}^{N} \frac{\beta_{i}}{\beta_{2}}\left(\frac{g_{i}}{g_{2}}\right)^{n} \mathbf{v}_{i}\right)_{j}\right| \\
& \sim\left|\beta_{2}\right|\left|g_{2}\right|^{n} \max _{j}\left|\mathbf{v}_{2, j}\right|
\end{aligned}
$$

where $\sim$ means 'is asymptotically equal to (as $n \rightarrow \infty)$ '.

Taking the logarithm of (3.4.48) gives

$$
\log \left(d_{\mathrm{PC}}(n T)\right) \sim n \log \left|g_{2}\right|+c,
$$

where $c$ is a constant given by

$$
c=\log \left|\beta_{2}\right|+\log \max _{j}\left|\mathbf{v}_{2, j}\right| \text {. }
$$

On the other hand $d_{\mathrm{PC}}(n)$ asymptotically defines the synchronization time by

$$
d_{\mathrm{PC}}(n T) \sim\left|\beta_{2}\right| \max _{j}\left|\mathbf{v}_{2, j}\right| \exp \left(-\frac{n T}{\tau_{\mathrm{PC}}}\right)
$$

which after taking the logarithm gives

$$
\log \left(d_{\mathrm{PC}}(n)\right) \sim c-\frac{n T}{\tau_{\mathrm{PC}}}
$$

with the constant $c$ from eq. 3.4.50, Comparing (3.4.49) with (3.4.52) leads to

$$
\tau_{\mathrm{PC}}=-\frac{T}{\log \left|g_{2}\right|}
$$

We numerically find the second largest eigenvalue of the matrix $G$ and use this to analytically calculate the synchronization time (3.4.53). To minimize the influence of specific rewired networks and perturbations we average over 100 realizations to obtain the average synchronization time $\left\langle\tau_{\mathrm{PC}}\right\rangle$. As for the Kuramoto system, the prediction of synchronization times based on the eigenvalues of the matrix $G$ well agrees with those obtained from direct numerical simulation (Fig. 3.5). 


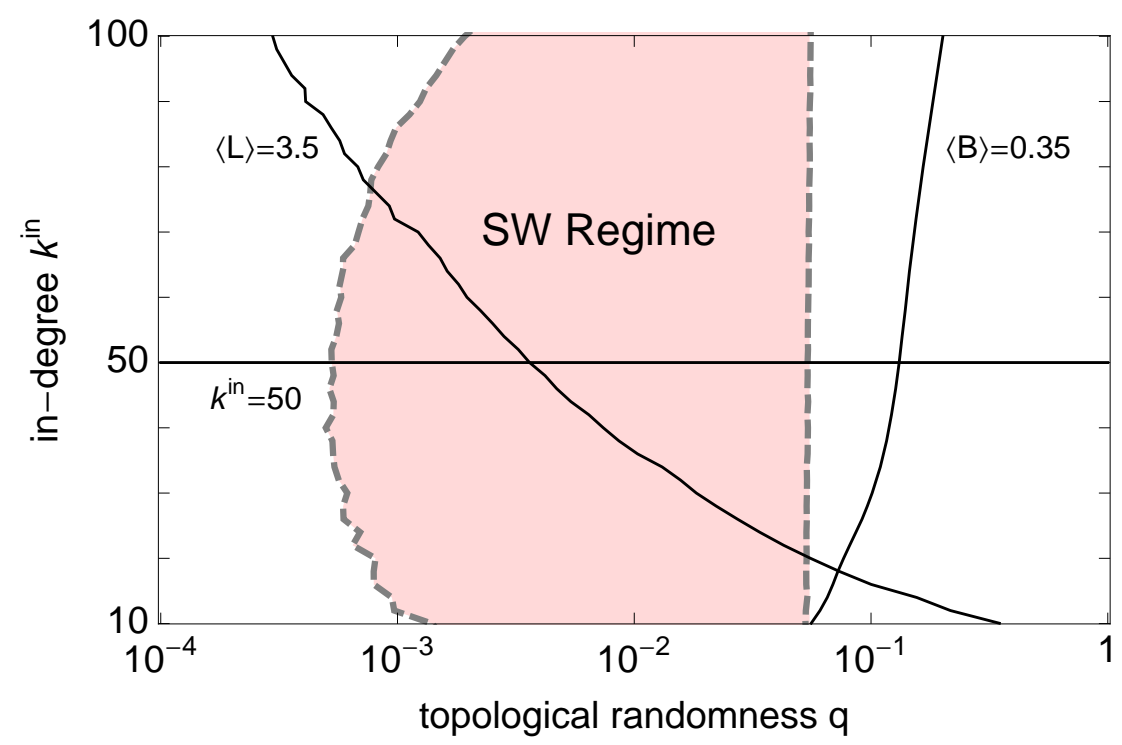

Figure 3.5: Comparing specific network ensembles. Solid lines indicate network ensembles with fixed in-degree $k^{i n}=50$, with fixed average path length $\langle L\rangle=3.5$ and with fixed betweenness centrality $\langle B\rangle=0.35$. The small-world regime (3.3.1) is located between the dashed lines.

\subsection{Synchronization time in dependence on topological randomness and degree}

In this Section we study the dependence of the synchronization time on the topological randomness $q$ and on the in-degree $k^{i n}$. Following the original approach of Watts and Strogatz we first examine ensembles with a fixed in-degree $k^{i n}$ and later generalize to generic network ensembles. As an overview, the localization of the different ensembles $k^{i n}(q)$ is depicted in Fig. 3.5.

\subsubsection{Choice of network ensembles}

We want to study the synchronization times with the emphasis on their behavior in the small-world regime which is defined by Eq. (3.3.1) and highlighted in Fig. 3.5 as the shaded region.

While the curves of the network ensembles with fixed in-degree $k^{i n}$ and those with fixed average path length $\langle L\rangle$ cross the small-world regime, this is not the case for the curves of the ensembles with fixed betweenness centrality $\langle B\rangle$ (see e.g. [Fre77], cf. also Chapter 2). However, we depicted the curve for these ensembles representatively to illustrate that one can not necessarily find curves of ensembles with a fixed topological observable that cross the small-world regime as desired. For instance, the same holds for the curves of network ensembles with fixed clustering coefficient $\langle C\rangle$.

To determine the synchronization times for these specific ensembles we use the derived analytical predictions in (3.4.5), (3.4.16) and (3.4.53), average them over 
100 network realizations and compare them to the synchronization times obtained via simulations. These results for the synchronization times are averaged over 100 realizations of networks and perturbations.

For the Kuramoto and pulse-coupled oscillators, determining the eigenvalues of the stability matrices of networks yields synchronization time estimates that agree well with those found from direct numerical simulations.

Only for the Rössler oscillators the synchronization times obtained from the numerical measurement of the decaying maximal distances (3.2.2) show small but systematic deviations from the analytically predicted ones. These deviations may be due to inaccuracies in determining the Euclidean distances that oscillate (see the decaying $x$-coordinates only in Fig. [3.1, b).

\subsubsection{Monotonicity: networks with fixed in-degree}

This section is dedicated to the dependence of average synchronization time $\left\langle\tau\left(q, k^{i n}\right)\right\rangle$ on the topological randomness $q$ for standard Watts-Strogatz ensembles of networks with fixed in-degree $k^{i n}$.

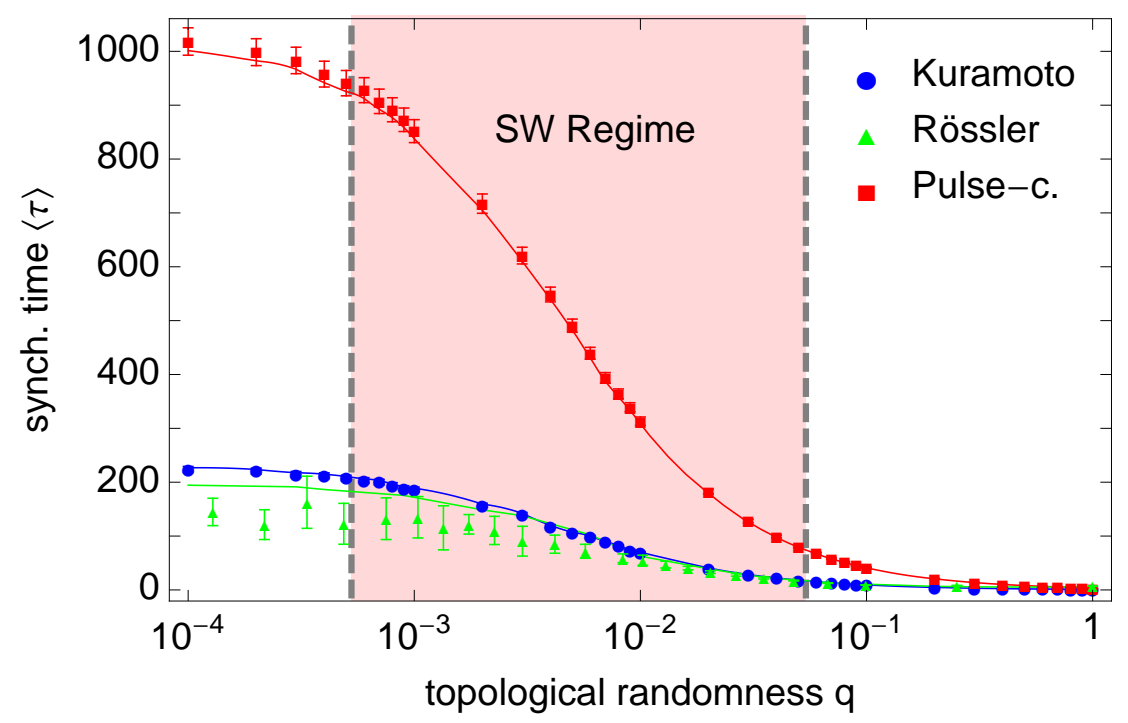

Figure 3.6: Monotonic dependence of the synchronization time $\langle\tau\rangle$ on the topological randomness $q$ for fixed in-degree $k^{i n}=50$. The small-world regime (3.3.1) is located in the shaded region between the dashed lines. Simulation results (symbols with standard deviations) for synchronization times of Kuramoto (blue, circle), Rössler (green, triangle) and pulse-coupled oscillators (red, square) in comparison to analytical predictions based on equations (3.4.4), (3.4.7) and (3.4.23) (solid lines), cf. also Fig. 3.5 for the localization of the network ensembles with fixed in-degree. 100 realizations were carried out in order to average over network topologies (and in simulations additionally over perturbations). Parameters as in Fig. 3.1. 
We see in Fig. 3.6 that $\left\langle\tau\left(q, k^{i n}\right)\right\rangle$ is monotonically decreasing with the topological randomness $q$ and systematically depends on the network topology: Regular ring networks $(q \rightarrow 0)$ are typically relatively slow to synchronize. We find that increasing $q$ towards the small-world regime induces shorter and shorter network synchronization times, with small worlds synchronizing a few times faster than regular rings. Further increasing the randomness $q$ induces even much faster synchronization, with fully random networks $(q \rightarrow 1)$ synchronizing fastest (two orders of magnitude faster than small worlds in our examples).

Thus, in network ensembles with fixed in-degree small worlds just occur intermediately during a monotonic increase of synchronization speed, but are not at all topologically optimal regarding their synchronization time.

One could try to explain this dependence heuristically by the decrease of the average characteristic path length. Indeed, the dependence of $\left\langle L\left(q, k^{i n}\right)\right\rangle$ on $q$ is also monotonically decreasing in a similar fashion. It is intuitive that as the characteristic path length decreases, oscillators can communicate more efficiently and this leads to faster and more efficient synchronization.

\subsubsection{Non-monotonicity: ensembles with fixed average path length}

We therefore systematically study the synchronization time for generalized WattsStrogatz ensembles of networks, specified by a function $k^{i n}(q)$, where the average path length $\langle L\rangle$ is fixed while the degree of randomness $q$ varies. We fix the average characteristic path length $\left\langle L\left(q, k^{i n}\right)\right\rangle=3.5$ as this gives us a wide range of $q$ values. However, the results below are not sensitive to a change of this specific value, cf. also GHGT10. We do not take $k^{i n}<10$ as the networks are in general no longer strongly connected for larger $q$ values. For each of these in-degrees $k^{i n}$ a value of the topopolgical randomness $q\left(k^{i n}\right)$ is determined. Note that the standard deviations are larger for smaller $q$ values. This is because we are rewiring a small number of edges here $\left(N k^{i n} / q\right.$ on average) and rewiring one edge more or less may have a strong effect on $L\left(q, k^{i n}\right)$ as it may add a long-range connection where there was not one previously. Note that $k^{i n}$ decreases in a non-linear fashion as $q$ increases for networks with $\left\langle L\left(q, k^{i n}\right)\right\rangle=3.5$. When we increase $q$, we decrease the indegree $k^{i n}$. Thus, it might be expected that the amount of coupling each oscillator receives also decreases. This would affect the synchronization time [TWG04]. We remove this factor by keeping the input each oscillator receives fixed (3.1.3) as $q$ varies. By doing this, we have reduced the effect of changing the in-degree $k^{i n}$ on the synchronization time. Surprisingly, the synchronization time of network ensembles with fixed average characteristic path length non-monotonically depends on the topological randomness (Fig. 3.7). In particular, networks with intermediate randomness in the small-world regime synchronize slowest.

\section{Chaotic Rössler oscillators}

We additionally investigate Rössler oscillators in the chaotic regime (see Section 2.2.2). Here, some difficulties arise (cf. also Section [3.4.2): 


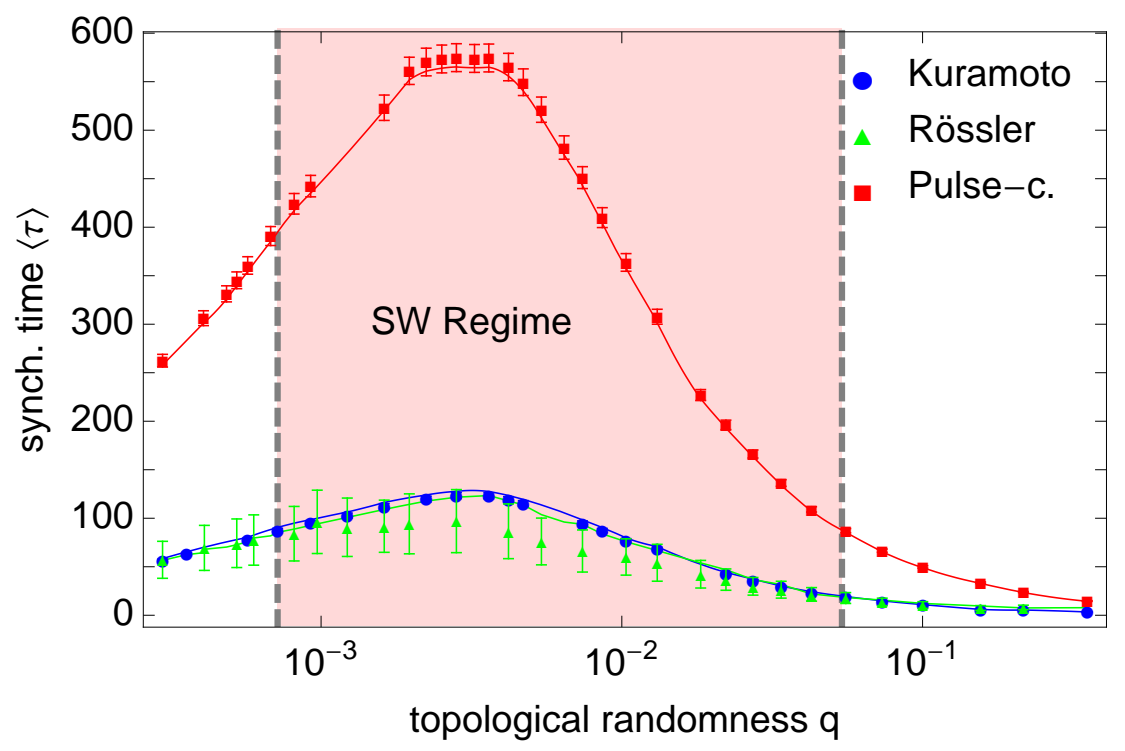

Figure 3.7: Non-monotonic dependence of the synchronization time $\langle\tau\rangle$ on the topological randomness $q$ with fixed average path length $\langle L\rangle=3.5$. The small-world regime (3.3.1) is located in the shaded region between the dashed lines. Simulation results (symbols with standard deviations) for synchronization times of Kuramoto (blue, circle), Rössler (green, triangle) and pulse-coupled oscillators (red, square) in comparison to analytical predictions based on equations (3.4.4), (3.4.7) and (3.4.23) (solid lines), cf. also Fig. 3.5 for the localization of the network ensembles with fixed average path length. 100 realizations were carried out in order to average over network topologies (and in simulations additionally over perturbations). Parameters as in Fig. 3.1,

In order to find an optimized value for the global coupling parameter $\sigma$ - encoded in $\Lambda$ according to (3.1.4) - that leads to synchronization, one notes that it is impossible to guarantee that each eigenvalue is located in the stable region, i.e. in the region where the master stability function takes only negative values (see Fig. 3.3). This is mainly due to the fact that the MSF for uncoupled chaotic Rössler oscillators is positive. This means that the minimal global coupling constant needed to achieve synchronization is always larger for the chaotic Rössler oscillators than for the periodic ones with the consequence that large eigenmodes may become unstable. In particular, this means that there is an upper limit on the size of a stable, synchronous array of chaotic Rössler oscillators [HCP94a, HPC95].

However, we numerically obtained results by only taking these network and perturbation realizations into account that lead to complete synchronization. For the chaotic Rössler oscillators as well, the synchronization time of network ensembles with fixed average characteristic path length non-monotonically depends on the topological randomness (Fig. 3.8). Still it remains an open task to confirm the synchronization times for the chaotic Rössler oscillators obtained numerically with 


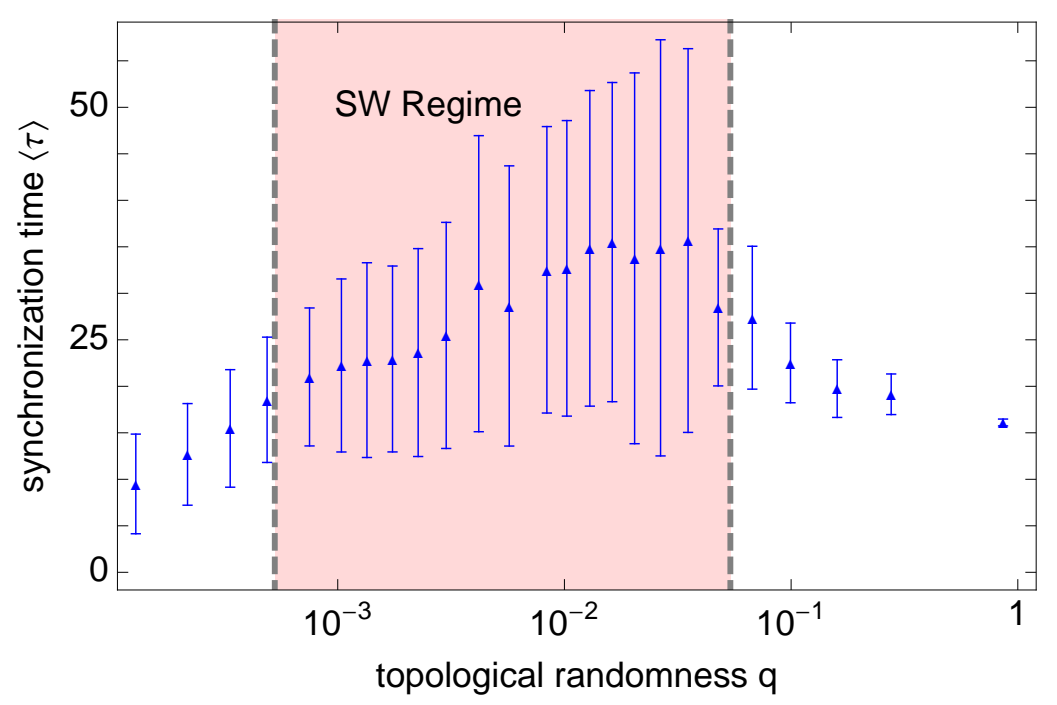

Figure 3.8: Simulations for chaotic Rössler oscillators indicate a nonmonotonic dependence of the synchronization time $\langle\tau\rangle$ on the topological randomness $q$ as well (fixed $\langle L\rangle=3.5$ ). The smallworld regime (3.3.1) is located in the shaded region between the dashed lines. Only simulation results are plotted. 100 realizations of synchronizing chaotic Rössler oscillators $(a=0.2, b=0.2, c=5.7, \sigma=6)$ were carried out in order to average over network topologies (and in simulations additionally over perturbations).

analytical predictions.

Since faster synchronization times are apparently not only related to the decrease of the average path length, we investigated the dependence on other topological observables which have been suggested to control whether or not a network actually synchronizes [NMLH03, LKJ06, GSKK06, MZK05b, ADGPV06, ZK06, $\mathrm{CHQ}^{+} 08$ ]. As already mentioned - in general - it is not possible to find curves of ensembles with a fixed topological observable that cross the small-world regime as desired.

Representatively, an ensemble with fixed betweenness centrality $B\left(k^{i n}, q\right)$ is shown in (Fig. 3.9). Curves of ensembles with fixed clustering coefficient or with one of the other mentioned topological observables show a similar dependence, but also cover no $q$ values in the small-world regime at all or only a small range.

\subsubsection{Generic nonlinear dependence of synchronization time on randomness and degree}

How does synchronization speed vary with randomness for more general ensembles $k^{i n}(q)$ ? A systematic study of the synchronization time as a function of both indegree $k^{i n}$ and randomness $q$ (Fig. 3.10) reveals an interesting nonlinear dependence.

Firstly, it confirms that for all networks with fixed in-degree $k^{i n}$ the synchronization time is monotonic in the randomness $q$ and the small-world regime at intermediate randomness is not specifically distinguished. 


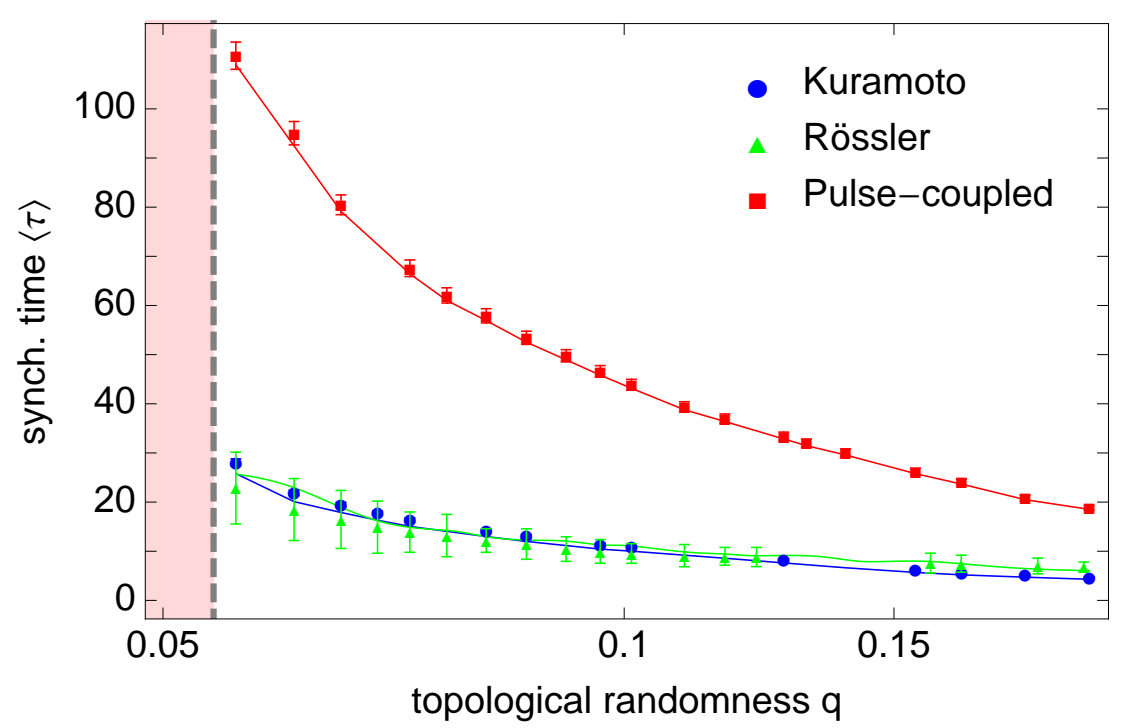

Figure 3.9: Monotonic dependence of the synchronization time $\langle\tau\rangle$ on the topological randomness $q$ with fixed betweenness centrality $\langle B\rangle=0.35$. Ensembles with fixed betweenness centrality do not cover any $q$ values in the small-world regime. Simulation results (symbols with standard deviations) for synchronization times of Kuramoto (blue, circle), Rössler (green, triangle) and pulse-coupled oscillators (red, square) in comparison to analytical predictions based on equations (3.4.4), (3.4.7) and (3.4.23) (solid lines), cf. also Fig. 3.5 for the localization of the network ensembles with fixed betweenness centrality. 100 realizations were carried out in order to average over network topologies (and in simulations additionally over perturbations). Parameters as in Fig. 3.1.

Secondly, the two-dimensional function $\left\langle\tau\left(q, k^{i n}\right)\right\rangle$ implies that ensembles of networks with fixed path lengths all exhibit a non-monotonic behavior of the synchronization time, with slowest synchronization for intermediate randomness.

Thirdly, considering graph ensembles characterized by any other smooth function $k^{i n}(q), q \in[0,1]$, shows that this is a general phenomenon and the specific choice of an ensemble $k^{i n}(q)$ is not essential.

In fact, as illustrated in Fig. 3.10, for any generic network ensemble $k^{\text {in }}(q)$ (including ensembles with fixed in-degree, fixed path length and fixed betweenness centrality as special choice (see Figures 3.5, 3.6, 3.7 and 3.9)) the synchronization speed $\left\langle\tau\left(q, k^{i n}(q)\right)\right\rangle$ is either intermediate or slowest, but never fastest at intermediate randomness, in particular in the small-world regime. It is remarkable that this seems to hold universally as the synchronization times are similar for Kuramoto oscillators (Fig. 3.10, a), periodic (Fig. 3.10, b) and chaotic GHGT10, Rössler oscillators and pulse-coupled oscillators (Fig. 3.10, c). All dynamical oscillator models considered show the same qualitative behavior, although the absolute values of the synchronization times differ from model to model due to the specific choice of the 
system parameters.

\subsubsection{Similarity between Kuramoto and pulse-coupled oscillators}

Comparing the synchronization times for Kuramoto and pulse-coupled oscillators in Fig. 3.10 both show a striking similarity. The question poses itself whether the synchronization times can be mapped on each other?

Therefore we will investigate the evolution of perturbations $\boldsymbol{\delta}(t)$ in both systems: For the Kuramoto oscillators we have

$$
\boldsymbol{\delta}_{\mathrm{K}}(t)=e^{\operatorname{Re} \lambda_{2} t} \boldsymbol{\delta}(0)
$$

while perturbations in the pulse-coupled system propagate like

$$
\boldsymbol{\delta}_{\mathrm{PC}}(n T)=\left|g_{2}\right|^{n} \boldsymbol{\delta}(0) .
$$

Obtaining similar synchronization times for both dynamics demands requires these perturbations to evolve in the same way. Setting $n T:=t$ the crucial eigenvalues should satisfy

$$
\operatorname{Re} \lambda_{2}=\frac{\log \left|g_{2}\right|}{T}
$$

Comparing the structure of the two relevant matrices $G$ (3.4.35) and $\Lambda$ (3.1.4) (see Fig. 3.11), we obtain the following relation for the respective eigenvalues $a_{2}$ and $\lambda_{2}$ :

$$
\lambda_{2}=\frac{c_{\mathrm{K}}}{c_{\mathrm{PC}}} g_{2}-1
$$

where the quotient $c_{\mathrm{K}} / c_{\mathrm{PC}}$ depends on the system parameters

$$
\begin{aligned}
c_{\mathrm{K}} & =\sigma_{\mathrm{K}} \\
c_{\mathrm{PC}} & =\frac{\gamma \sigma_{\mathrm{PC}}}{I e^{-\gamma \Delta}+\gamma \sigma_{\mathrm{PC}}} .
\end{aligned}
$$

Note that $\sigma_{\mathrm{K}}>0$ while $\sigma_{\mathrm{PC}}<0$. Setting the parameters in the way that this quotient and the period $T$ ( $T$ is close to one anyhow) are both equal to one, we get

$$
1+\log \left|g_{2}\right|=\operatorname{Re} g_{2} \quad \text { provided }\left|g_{2}\right| \approx 1, \operatorname{Re} g_{2} \approx 1 .
$$

This means that synchronization times obtained for networks of pulse-coupled oscillators and for the same structured networks of Kuramoto oscillators are equal if the second largest eigenvalue of matrix $A$ satisfies $\operatorname{Re} g_{2} \approx 1$ and $\left|g_{2}\right| \approx 1$, in the sense that $|\log | g_{2}|| \ll 1$. This is in general the case for large networks, for which the stochastic matrix $A$ has $N$ eigenvalues with real parts in $[-1,1]$. 

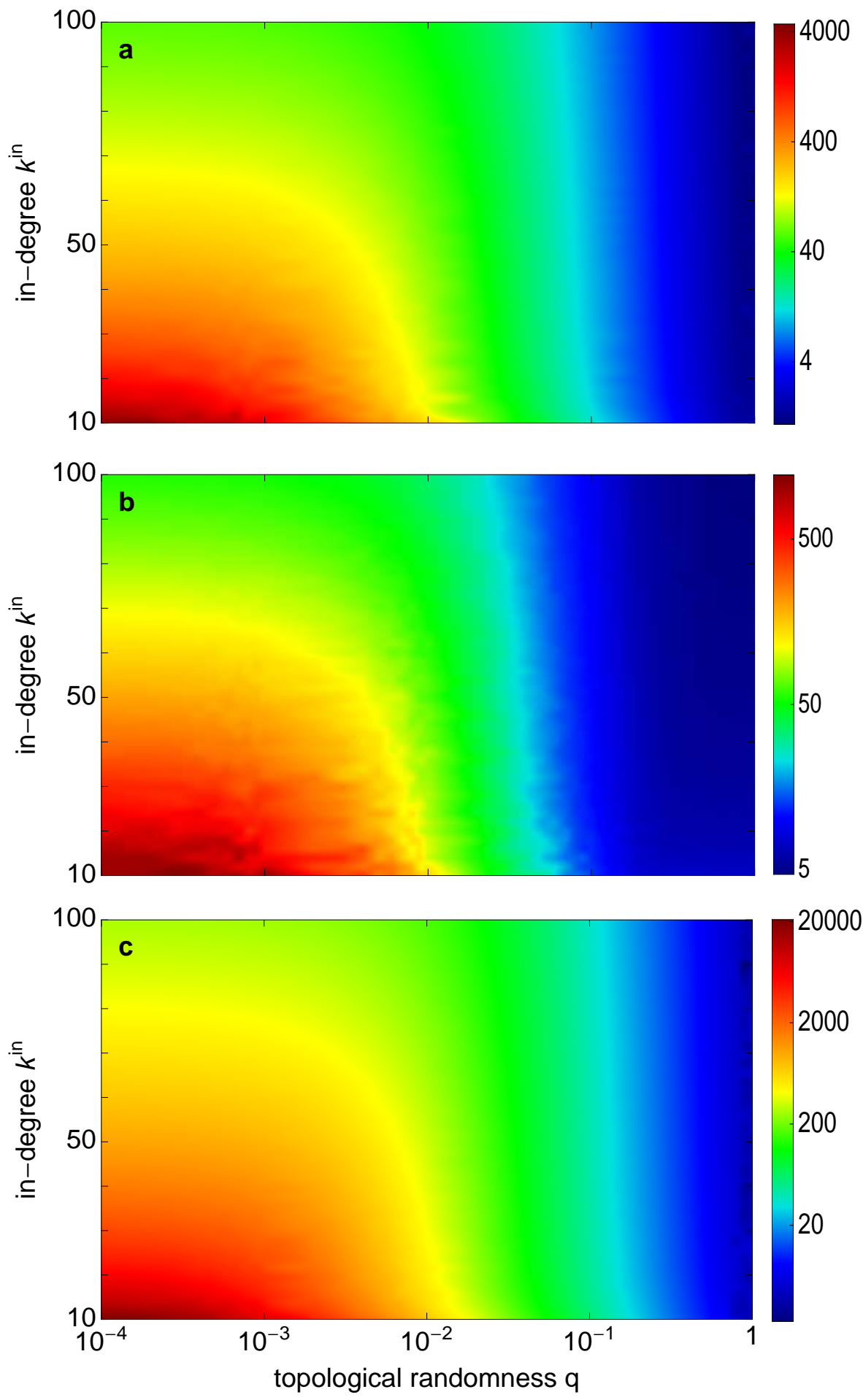

Figure 3.10: Universal nonlinear dependence of synchronization time on in-degree $k^{i n}$ and topological randomness $q$. No generic ensemble $k^{i n}(q)$ exhibits fastest synchronization in the small-world regime. a: Kuramoto oscillators, b: Rössler oscillators, c: pulse-coupled oscillators; logarithmic color scales. Parameters chosen as in Fig. 3.1. Synchronization times are obtained from equations (3.4.5), (3.4.16) and (3.4.53), cf. also Fig. 3.5 for the localization of the specific network ensembles studied in previous sections. (modified from GHGT10, GGT11) 


$$
\left.\begin{array}{ccccccc}
1-c_{\mathrm{PC}} & 0 & 0 & \frac{c_{\mathrm{PC}}}{2} & \frac{c_{\mathrm{PC}}}{2} & 0 \\
\frac{c_{\mathrm{PC}}}{2} & 1-c_{\mathrm{PC}} & 0 & \frac{c_{\mathrm{PC}}}{2} & 0 & 0 \\
0 & \frac{c_{\mathrm{PC}}}{2} & 1-c_{\mathrm{PC}} & 0 & 0 & \frac{c_{\mathrm{PC}}}{2} \\
\frac{c_{\mathrm{PC}}}{2} & 0 & \frac{c_{\mathrm{PC}}}{2} & 1-c_{\mathrm{PC}} & 0 & 0 \\
0 & 0 & \frac{c_{\mathrm{PC}}}{2} & 0 & 1-c_{\mathrm{PC}} & \frac{c_{\mathrm{PC}}}{2} \\
0 & \frac{c_{\mathrm{PC}}}{2} & 0 & 0 & \frac{c_{\mathrm{PC}}}{2} & 1-c_{\mathrm{PC}}
\end{array}\right)
$$

Figure 3.11: Comparing the structures of the two relevant matrices $G$ (3.4.35) and $\Lambda$ (3.1.4) (Cartoon for $N=6$ and $k^{i n}=2$ ). The constants $c_{\mathrm{PC}}$ and $c_{\mathrm{K}}$ depend on the parameters and are given by eqs. (3.5.6) and (3.5.5) 


\subsection{Real-world networks}

original network

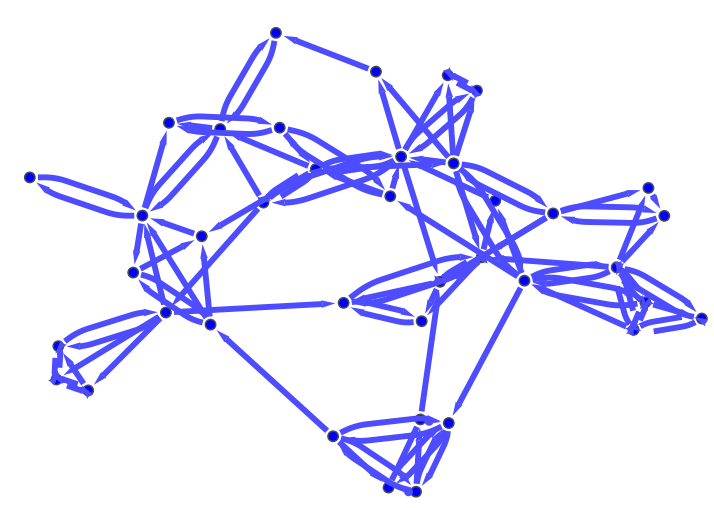

$\mathrm{p}=0$ totally randomized network

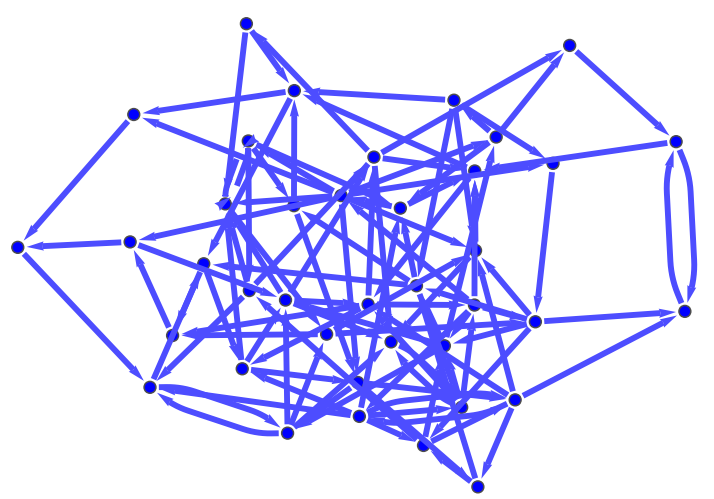

$\mathrm{p}=1$

Figure 3.12: Randomizing real-world networks. Depicted is a social network (inmates in prison $\left.\left[\mathrm{VDHS}^{+} 03\right]\right)$ : the original one $(p=0)$ in comparison to its totally randomized network $(p=1)$.

For Watts-Strogatz small-world model networks we have found that the synchronization speed is either intermediate or slowest, but never fastest in the small-world regime. Moreover, keeping the in-degree fixed, the model networks synchronize the faster the more random they are. To support that this monotone relation also holds in much more generality we considered various real-world networks: an air transport network [CPSV07], a social network [VDHS ${ }^{+}$03], a neural network [AY92, an organisational network [CP04] and a human travel network [BHG06].

\subsubsection{Randomizing real-world networks}

Randomization is performed by rewiring the existing connections with a probability $p \in[0,1]$. Note that the rewiring probability $p$ and the topological randomness $q$ are two different quantities. Here we start with the original real-world network $(p=0)$, which may be in the small-world regime already. The rewiring process is performed as explained in Section 2.2.1: only the outgoing edges of the directed links are rewired, which means that each node's in-degree stays constant during the rewiring. Having considered networks with the same in-degrees for all nodes so far, these in-degrees may vary in real-world networks: But due to the rewiring routine the network's initial in-degree distribution is kept constant during rewiring. Thus it is not uncommon for a single strongly-connected component (SCC) network to split to several ones [Tim06]. If splitting occurs the rewiring is repeated until the resulting network consists of one SCC only again. All measured quantities are averaged over 100 network realizations. 

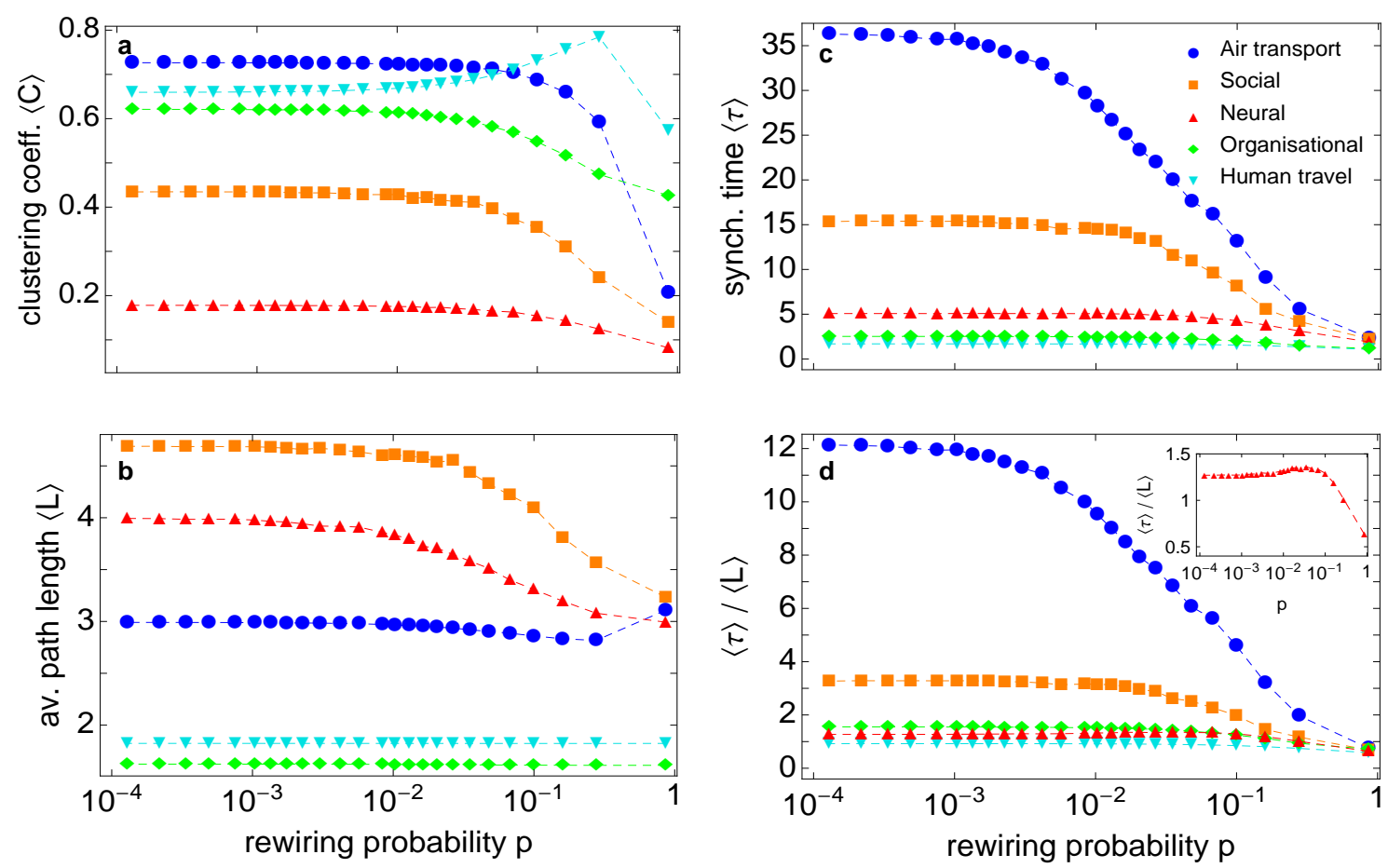

Figure 3.13: Real-world networks consistently synchronize several times slower than their randomized counterparts (air transport network: the US airports with the largest amount of traffic [CPSV07, social network: inmates in prison [VDHS $\left.{ }^{+} 03\right]$, neural network: $C$. Elegans [AY92], organisational network: research team in a manufacturing company [CP04, human travel network: based on the trajectories of dollar bills [BHG06]). a: Clustering coefficients $\langle C\rangle$. b: Average shortest path lengths $\langle L\rangle$. c: Synchronization times $\langle\tau\rangle$. d: Synchronization times $\langle\tau\rangle$ relative to the average path length $\langle L\rangle$. Inset: the synchronization times for $C$. Elegans divided by the average path length $\langle L\rangle$ show slightly non-monotonic behavior. All quantities are averaged over 100 network realizations. Dashed lines are only a guide to the eye.

\subsubsection{Randomized real-world networks synchronize faster}

The studied real-world networks show small-world behavior: gradually randomizing these networks, their clustering coefficients and their average path lengths monotonically decrease (Fig. 3.13, a and b). Only the clustering coefficient of the human travel network partly shows a non-monotonic behaviour. This network is extremely large (464670 nodes) in comparison to the other networks ( $\approx 100-500$ nodes).

We found that all real-world networks consistently synchronized several times slower than their randomized counterparts (Fig. 3.13, c). For all networks, the synchronization times monotonically increase with increasing random rewiring. For all but the neural circuit of $C$. Elegans AY92, this holds even for ensembles with fixed average path length; for the latter we observed non-monotonic dependence 
with slowest synchronization for intermediate randomness (Fig. 3.13, d).

\subsection{Summary and discussion}

In this Chapter we investigated the effect of topology on the speed of synchronization for various oscillator types, intrinsic dynamics and coupling schemes: phase oscillators coupled via phase differences, higher-dimensional periodic systems coupled diffusively as well as neural circuits with inhibitory delayed pulse-coupling and consistently found qualitatively the same results. We derived analytical predictions for the asymptotic synchronization times, including an extension of the master stability function to determine how fast the system actually synchronizes. We compared the synchronization speed for different network ensembles:

Firstly, we found that for networks of fixed in-degree $k$, the average synchronization time $\langle\tau\rangle$ is monotonically decreasing with the topological randomness $q$. Comparing different fixed- $k$ ensembles small-world networks always synchronize quicker than regular networks.

Secondly, the intuitive idea that this is due to the decrease in average characteristic path length $\langle L(q, k)\rangle$ does not provide a complete explanation: Instead of fixing the in-degree, we fixed the average characteristic path length. For such ensembles networks in the small-world regime synchronize slower than regular networks and the synchronization speed non-monotonically depends on the topological randomness $q$. The in-degree $k$ is monotonically decreasing with $q$ and so does the clustering coefficient $\langle C(q, k)\rangle$. So neither of these topological properties alone gives an obvious explanation and the phenomenon results from an interplay between several network properties. For example, the faster synchronization of regular networks than of small-world networks may be due to the in-degree $k$ being large. This is not because the total coupling strength $J_{i j}=k \sigma$ is high, as we kept this fixed for all $(k, q)$-pairs, but may be because the oscillators receive the coupling effect from a large number of oscillators. However, we also see fast synchronization for random networks where $k$ is small and so the same total coupling amount is received from far fewer interacting oscillators. So the explanation for the non-monotonic dependence is non-trivial.

Thirdly, we investigated the dependence on other topological observables apart from small-world properties: network ensembles with fixed betweenness centrality have been displayed as an example, but yet a simple explanation for the nonlinear dependence is missing.

Furthermore, we studied the full nonlinear dependence of the synchronization time on the in-degree $k$ and the topological randomness $q$ for generic network ensembles. We found that fastest synchronization is essentially impossible in the small-world regime, except for highly artificial ensembles. This statement holds for all observed dynamics. In particular, the synchronization times for the Kuramoto and pulsecoupled oscillators are strikingly similar.

It would be interesting to extend the analysis started in Section 3.5.5 to find out under which conditions the synchronization times for Kuramoto oscillators could be approximated by or even analytically mapped on the times for the pulse-coupled 
oscillators.

We found that small worlds in general never synchronize fastest. Specifically, in networks with fixed average path length, synchrony is consistently fast for regular rings, fastest for completely random networks, and slowest in the intermediate smallworld regime (Fig. 3.5). It is an astonishing result that this holds across various oscillator types, intrinsic dynamics and coupling schemes: phase oscillators coupled via phase differences, higher-dimensional periodic systems coupled diffusively as well as neural circuits with inhibitory delayed pulse-coupling.

In particular, small-world topologies are not at all special and may synchronize orders of magnitude slower than completely random networks. So generically the small-world regime either exhibits slowest synchronization or just exhibits no extremal properties regarding synchronization times.

Given the variety of the investigated dynamical systems our results indicate that this is a universal phenomenon.

Our investigations of real-world networks support this view. Although the considered networks may be in the small-world regime already, rewiring still strongly increases the synchronization speed, even for ensembles with fixed average path length. It remains an open question why rewiring typically implies faster synchronization.

In this Chapter we focused on systems with fixed size $N$. In the Watts-Strogatz ensemble the scaling of quantities such as the average path length $L$ or the clustering coefficient $C$ with the system size depends heavily on $q$ [WS98], e.g. $L \sim N$ for $q=0$ and $L \sim \log N$ for $q=1$. Therefore, it would be an interesting question to study how the results illustrated in Fig. 3.10 change with $N$ and what would be the appropriate definition of the small-world regime and other generalized ensembles with given structural features as a function of the system size.

Additionally an understanding or even an analytical description of the curves of same synchronization times in Fig. 3.10 is extremely helpful for finding further relations between the topology and the dynamics of complex networks. In particular, it is an interesting question to understand the behavior of the second largest eigenvalue of the Laplacian as a function of $q$ for fixed $k$ and $N$.

We address these questions - amongst others - in the next Chapter 4 . 


\section{Small-World Spectra in Mean Field Theory}

In this Chapter we derive analytic expressions for the small-world spectra based on a two-stage mean field approximation that we introduce. A single formula covers the entire spectrum from regular via small-world to strongly randomized topologies, explaining also the simultaneous dependencies on network size $N$, average degree $k$ and randomness $q$. Numerical diagonalization of Laplacians of undirected and directed networks shows that the analytic prediction well approximates all actual eigenvalues, except for extremal parameter settings such as $q$ of the order of unity, where standard Random Matrix Theory can be applied.

Small-world models based on rewiring have received widespread attention both theoretically and in applications, as demonstrated by the huge number of references pointing to the original work WS98. But for most of their features analytical predictions are not known to date ([BW00]; for a mean field solution of the average path length see [NMW00]). In particular, the spectrum of small-world Laplacians has been studied for several specific cases and numerically Mon99, JJ01, BP02, MO04, KvM11, but a general derivation of reliable analytic predictions is still missing.

In general, the study of the graph Laplacian is extremely important since the Laplacian is intimately connected to the structure of its underlying network (see e.g. [Bol98, BL07]). Therefore, a broad area of research is related to its study and in particular, to the study of its spectrum [MZK05b, Chu05, MZK05a, AC05, DNM06, SDM08, BJ08, MM08, (cf. also Chapter 2).

The structural features of complex networks - encoded in the graph Laplacian underly their collective dynamics such as synchronization, diffusion, relaxation and coordination processes [PRK01, Str01]. Such processes occur in various fields ranging from opinion formation in social networks [PLR05] and consensus dynamics of agents OS05 to synchronization in biological circuits [BCDLR10, MMZ04] and relaxation oscillations in gene regulatory networks [McM02, GdBLC03]. In particular, the asymptotic dynamics is characterized by the graph Laplacian.

This Chapter is organized as follows. In Section 4.1 we first present rewiring 'on average', a new mean field rewiring proposed by us. Based on this rewiring, we derive a single formula that covers the entire spectrum from regular to strongly randomized topologies in Section 4.2. Then we investigate the ordering of the mean field eigenspectrum in Section 4.3, in particular, whether the index $l=2$ is always related to the second-largest eigenvalue. In Section 4.4 we investigate the accuracy of our predictions via systematic numerical checks for the extreme eigenvalues. For the topological randomness $q$ of the order of unity, standard Random Matrix Theory helps out in Section 4.5. Finally, we study the entire spectrum in Section 4.4. We 

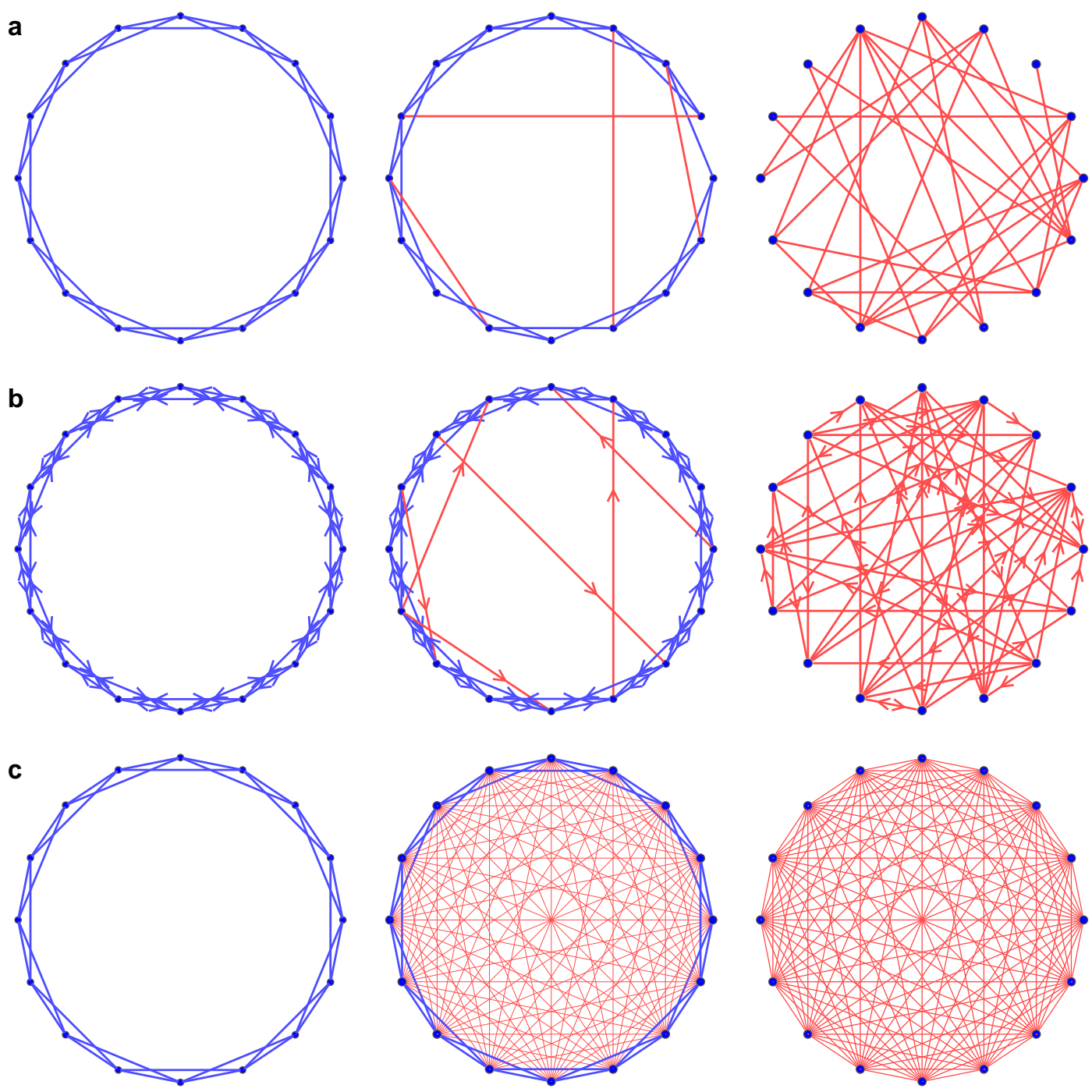

Figure 4.1: Rewiring 'on average'. (Cartoon for $N=16$ and $k=4$ ) Single realizations of rewiring for (a) undirected and (b) directed networks; (c) mean field rewiring. From left to right: $q=0$ (regular ring network), $q=0.1$ ('small world') and $q=1$ (random network). The regular ring network is the same for (a), (b) and (c). Adapted from GGT12.

close in Section 4.7 with a summary and a discussion of further work.

\subsection{A new mean field rewiring}

Consider a graph of $N$ nodes on a one-dimensional ring lattice with periodic boundary conditions. Each node receives links from its $k / 2$ nearest neighbors on both sides ( $k$ is chosen to be even). Randomness is introduced by rewiring. Following [WS98] for undirected networks, we first cut each edge with probability $q$. Afterwards the cut edges are rewired to nodes chosen uniformly at random from the whole network 
(avoiding double edges and self-loops). Similarly, for directed [Fag07 networks, we first cut all out-going edges with probability $q$ and rewire their tips afterwards (avoiding double edges and self-loops as well).

The generic asymptotic relaxation dynamics on such a network is characterized by its graph Laplacian $\Lambda$ (see also equations (2.1.21) and (2.1.22) $)$ defined by its elements

$$
\Lambda_{i j}=A_{i j}\left(1-\delta_{i j}\right)-k_{i} \delta_{i j}
$$

for $i, j \in\{1, \ldots, N\}$, where $A_{i j}$ are the elements of the adjacency matrix (one for an existing edge and zero for no edge), $k_{i}$ is the degree of node $i$ (replaced by the in-degree for directed networks) and $\delta_{i j}$ is the Kronecker-delta. Note that the spectrum of the Laplacian for a directed network is complex while the spectrum for an undirected one is real.

\subsection{Derivation of an analytical expression for the entire small-world spectrum}

To analytically predict the Laplacian spectrum of the network models in dependence of the network size $N$, the average degree $k$ and the topological randomness $q$, we introduce 'rewiring on average', as depicted in Fig. 4.1 in comparison to both other rewiring procedures for undirected and directed networks. First we define a circulant mean field Laplacian

$$
\tilde{\Lambda}^{\mathrm{mf}}=\left(\begin{array}{cccccc}
c_{0} & c_{1} & c_{2} & & \cdots & c_{N-1} \\
c_{N-1} & c_{0} & c_{1} & c_{2} & & \vdots \\
& c_{N-1} & c_{0} & c_{1} & \ddots & \\
\vdots & & \ddots & \ddots & \ddots & c_{2} \\
& & & & & c_{1} \\
c_{1} & \cdots & & & c_{N-1} & c_{0}
\end{array}\right) .
$$

For the initial ring $(q=0)$, Eq. (4.2.1) is exact and the matrix elements take the form

$$
c_{i}= \begin{cases}-k & \text { if } i=0 \\ 1 & \text { if } i \in\left\{1, \ldots, \frac{k}{2}, N-\frac{k}{2}, \ldots, N-1\right\}=S_{1} \\ 0 & \text { if } i \in\left\{\frac{k}{2}+1, \ldots, N-\frac{k}{2}-1\right\}=S_{2}\end{cases}
$$

where we classify the elements into those representing the original ring $S_{1}$ and those representing absent edges $S_{2}$ outside that ring.

For given $q>0$, instead of rewiring each edge independently with a certain probability to obtain a specific randomized network, we now 'rewire on average' to obtain a mean field version of the randomized network ensemble: Firstly, the average total weight $q k N / 2$ representing all edges to be rewired is subtracted uniformly from the weights of existing edges of $S_{1}$. Secondly, the rewired weight is distributed uniformly among the total 'available' weight in the whole network. The latter is given by

$$
f=\frac{N(N-1)-(1-q) k N}{2}
$$


where each edge is assumed to carry at most weight one. Of this total, the weight

$$
f_{1}=\frac{q k N}{2}
$$

is available in $S_{1}$ and

$$
f_{2}=\frac{N(N-1)-k N}{2}
$$

in $S_{2}$. The fraction $f_{1} / f$ is then assigned to elements representing edges in $S_{1}$ and the fraction $f_{2} / f$ to those representing $S_{2}$. Therefore, an individual edge in $S_{1}$ gets the additional weight

$$
w_{1}=\frac{f_{1}}{f} \frac{\frac{q k N}{2}}{\frac{k N}{2}}=\frac{q^{2} k}{N-1-(1-q) k},
$$

and an edge in $S_{2}$ gets the new weight

$$
w_{2}=\frac{f_{2}}{f} \frac{\frac{q k N}{2}}{\frac{N(N-1)-k N}{2}}=\frac{q k}{N-1-(1-q) k} .
$$

Thus, in our mean field theory the elements of the Laplacian (4.2.1) of a network on $N$ nodes with degree $k$ after rewiring with probability $q$ are given by

$$
c_{i}= \begin{cases}-k & \text { if } i=0 \\ 1-q+w_{1} & \text { if } i \in S_{1} \\ w_{2} & \text { if } i \in S_{2} .\end{cases}
$$

The mean field Laplacian defined by (4.2.1) and (4.2.8) by construction is a circulant matrix with eigenvalues [GVL96, LT85, Gra01]

$$
\tilde{\lambda}_{l}^{\mathrm{mf}}=\sum_{j=0}^{N-1} c_{j} \exp \left(\frac{-2 \pi \mathrm{i}(l-1) j}{N}\right) .
$$

Observing the structure in Fig. 4.2 we immediately obtain the trivial eigenvalue for $l=1$ :

$$
\begin{aligned}
\tilde{\lambda}_{1}^{\mathrm{mf}} & =\left.\sum_{j=0}^{N-1} c_{j} \exp \left(\frac{-2 \pi \mathrm{i}(l-1) j}{N}\right)\right|_{l=1} \\
& =\sum_{j=0}^{N-1} c_{j}=-k+k\left(1-q+w_{1}\right)+(N-k-1) w_{2}=0,
\end{aligned}
$$

which is common to all networks (for all $q, N$, and any $k \leq N-1$ ) and reflects the invariance of Laplacian dynamics against uniform shifts, as seen from the associated eigenvector $\tilde{v}_{1}=(1, \ldots, 1)^{\top}$.

To obtain the remaining eigenvalues for $l \in\{2, \ldots, N\}$ we first define

$$
x_{l}:=\exp \left(\frac{-2 \pi \mathrm{i}(l-1)}{N}\right) \text {, }
$$




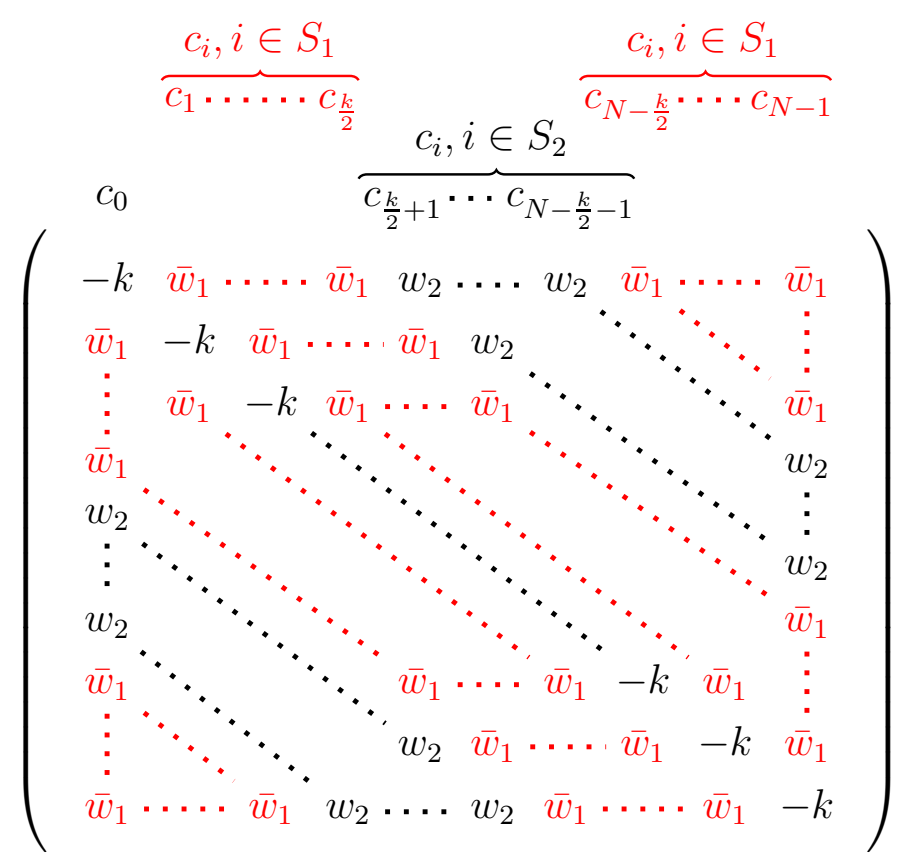

Figure 4.2: The banded structure of the mean field graph Laplacian. It has the weights $\bar{w}_{1}=1-q+w_{1}$ for $c_{i} \mid i \in S_{1}$ and $w_{2}$ for $c_{i} \mid i \in S_{2}$. For $q=0$ and hence $\bar{w}_{1}=1$ and $w_{2}=0$ the exact ring Laplacian is reobtained.

$$
c^{\prime}:=\frac{1-q}{k}+q c^{\prime \prime}
$$

and

$$
c^{\prime \prime}:=\frac{q}{N-1-(1-q) k}
$$

This leads to

$$
\begin{aligned}
\tilde{\lambda}_{l}^{\mathrm{mf}} & =-k+k c^{\prime} \sum_{j=1}^{\frac{k}{2}} x_{l}^{j}+k c^{\prime \prime} \sum_{j=\frac{k}{2}+1}^{N-1-\frac{k}{2}} x_{l}^{j}+k c^{\prime} \sum_{j=N-\frac{k}{2}}^{N-1} x_{l}^{j} \\
& =-k+k c^{\prime} \sum_{j=1}^{\frac{k}{2}} x_{l}^{j}+k c^{\prime} \sum_{j=1}^{\frac{k}{2}} x_{l}^{N-j}+k c^{\prime \prime}\left(\sum_{j=1}^{\frac{N}{2}-\frac{k}{2}-1} x_{l}^{\frac{N}{2}+j}+\sum_{j=1}^{\frac{N}{2}-\frac{k}{2}-1} x_{l}^{\frac{N}{2}-j}+x_{l}^{\frac{N}{2}}\right)
\end{aligned}
$$

where we have exploited the additional transposition symmetry $\tilde{\Lambda}^{\mathrm{mf}}=\left(\tilde{\Lambda}^{\mathrm{mf}}\right)^{\top}$ which implies $c_{j}=c_{N-j}$. Applying the Euler formula GR07.

$$
\exp (\mathrm{i} \alpha)=\cos (\alpha)+\mathrm{i} \sin (\alpha),
$$


the complex summands cancel and we get

$$
\tilde{\lambda}_{l}^{\mathrm{mf}}=-k+2 k c^{\prime} \sum_{j=1}^{\frac{k}{2}} \cos \left(\frac{2 \pi(l-1) j}{N}\right)+x_{l}^{\frac{N}{2}} k c^{\prime \prime}\left(2 \sum_{j=1}^{\frac{N}{2}-\frac{k}{2}-1} \cos \left(\frac{2 \pi(l-1) j}{N}\right)+1\right) .
$$

Furthermore, applying the trigonometric identity GR07

$$
\begin{aligned}
\sum_{j=0}^{n} \cos (j \alpha) & =\cos \left(\frac{n+1}{2} \alpha\right) \sin \left(\frac{n \alpha}{2}\right) \frac{1}{\sin \left(\frac{\alpha}{2}\right)}+1 \\
& =\cos \left(\frac{n \alpha}{2}\right) \sin \left(\frac{n+1}{2} \alpha\right) \frac{1}{\sin \left(\frac{\alpha}{2}\right)} \\
& =\frac{1}{2}\left(1+\frac{\sin \left(\left(n+\frac{1}{2}\right) \alpha\right)}{\sin \left(\frac{\alpha}{2}\right)}\right),
\end{aligned}
$$

yields

$$
\tilde{\lambda}_{l}^{\mathrm{mf}}=-k+k c^{\prime}\left(\frac{\sin \left(\frac{(k+1)(l-1) \pi}{N}\right)}{\sin \left(\frac{(l-1) \pi}{N}\right)}-1\right)+x_{l}^{\frac{N}{2}} k c^{\prime \prime} \frac{\sin \left(\frac{(N-k-1)(l-1) \pi}{N}\right)}{\sin \left(\frac{(l-1) \pi}{N}\right)}
$$

Taking advantage of additional identities - only valid for $l \in \mathbb{Z}$ (Fig. 4.3) -

$$
\begin{gathered}
x_{l}^{N / 2}=(-1)^{l-1}, \\
(-1)^{l-1} \sin (\alpha)=\sin (\alpha+(l-1) \pi)
\end{gathered}
$$

and the symmetry

$$
\sin (-\alpha)=-\sin (\alpha),
$$

the expression simplifies to

$$
\begin{aligned}
\tilde{\lambda}_{l}^{\mathrm{mf}} & =-k+k c^{\prime}\left(\frac{\sin \left(\frac{(k+1)(l-1) \pi}{N}\right)}{\sin \left(\frac{(l-1) \pi}{N}\right)}-1\right)+(-1)^{l-1} k c^{\prime \prime} \frac{\sin \left(\frac{(-(k+1)+N)(l-1) \pi}{N}\right)}{\sin \left(\frac{(l-1) \pi}{N}\right)} \\
& =-k+k c^{\prime}\left(\frac{\sin \left(\frac{(k+1)(l-1) \pi}{N}\right)}{\sin \left(\frac{(l-1) \pi}{N}\right)}-1\right)+k c^{\prime \prime} \frac{\sin \left(\frac{(-(k+1)(l-1)+2 N(l-1)) \pi}{N}\right)}{\sin \left(\frac{(l-1) \pi}{N}\right)} \\
& =-k+k c^{\prime}\left(\frac{\sin \left(\frac{(k+1)(l-1) \pi}{N}\right)}{\sin \left(\frac{(l-1) \pi}{N}\right)}-1\right)-k c^{\prime \prime} \frac{\sin \left(\frac{(k+1)(l-1) \pi}{N}\right)}{\sin \left(\frac{(l-1) \pi}{N}\right)} \\
& =-k-k c^{\prime}+k\left(c^{\prime}-c^{\prime \prime}\right) \frac{\sin \left(\frac{(k+1)(l-1) \pi}{N}\right)}{\sin \left(\frac{(l-1) \pi}{N}\right)}
\end{aligned}
$$

for $l \in\{2, \ldots, N\}$. 


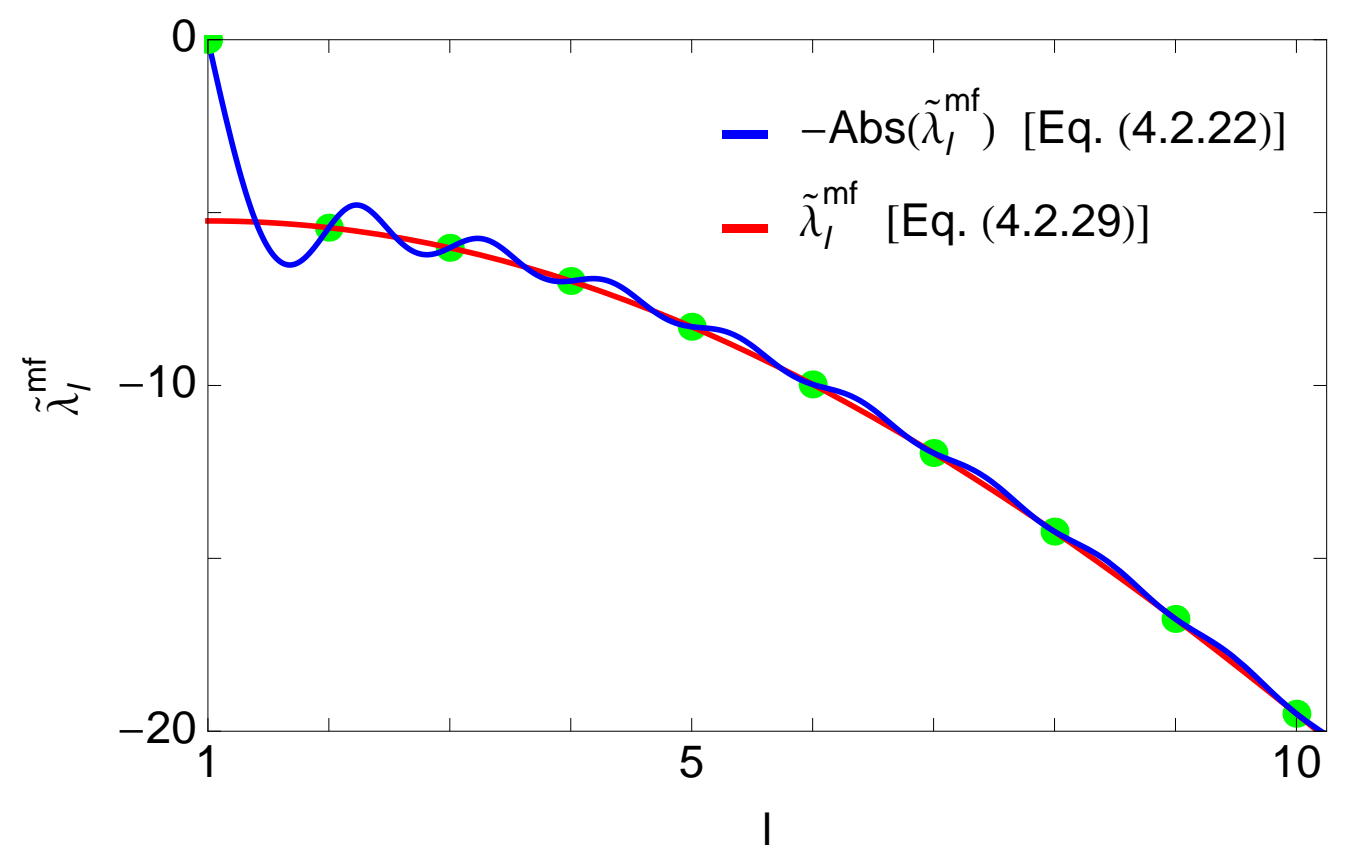

Figure 4.3: Interpolating the eigenvalues. Eqs. (4.2.22) and (4.2.29) both contain the eigenspectrum $\tilde{\lambda}_{l}^{\mathrm{mf}}$ for $l \in\{2, \ldots, N\}$ correctly (green circles). But while $\tilde{\lambda}_{l}^{\mathrm{mf}}$ in Eq. (4.2.22) includes $\tilde{\lambda}_{1}^{\mathrm{mf}}$ as $\lim _{l \rightarrow 1} \tilde{\lambda}_{l}^{\mathrm{mf}}=\tilde{\lambda}_{1}^{\mathrm{mf}}=0$ as well, $\tilde{\lambda}_{l}^{\mathrm{mf}}$ in Eq. (4.2.29) does not: To further simplify expressions, we have used identities (4.2.23) and (4.2.24) only valid for $l \in \mathbb{Z}$.

\subsection{The ordering of the mean field spectrum}

The spectrum obeys the symmetry

$$
\tilde{\lambda}_{l}^{\mathrm{mf}}=\tilde{\lambda}_{N-l+2}^{\mathrm{mf}},
$$

but is unordered otherwise, i.e. the index $l$ does neither denote eigenvalues with decreasing real part as for the eigenvalues $\lambda_{i}$ of the graph Laplacian $\Lambda$ (3.1.4) nor eigenvalues with decreasing absolute value as for the eigenvalues $g_{i}$ of $G$ (3.4.35).

As we argue below the expression $\tilde{\lambda}_{2}^{\mathrm{mf}}\left(=\tilde{\lambda}_{N}^{\mathrm{mf}}\right.$ due to (4.3.1) ) always constitutes the second largest eigenvalue. The only term depending on $l$ in eq. (4.2.29) is the ratio

$$
\frac{\sin \left(\frac{(k+1)(l-1) \pi}{N}\right)}{\sin \left(\frac{(l-1) \pi}{N}\right)} .
$$

We therefore study the function

$$
f(x)=\frac{\sin ((k+1) x)}{\sin x}
$$

with

$$
x=\frac{(l-1) \pi}{N}
$$




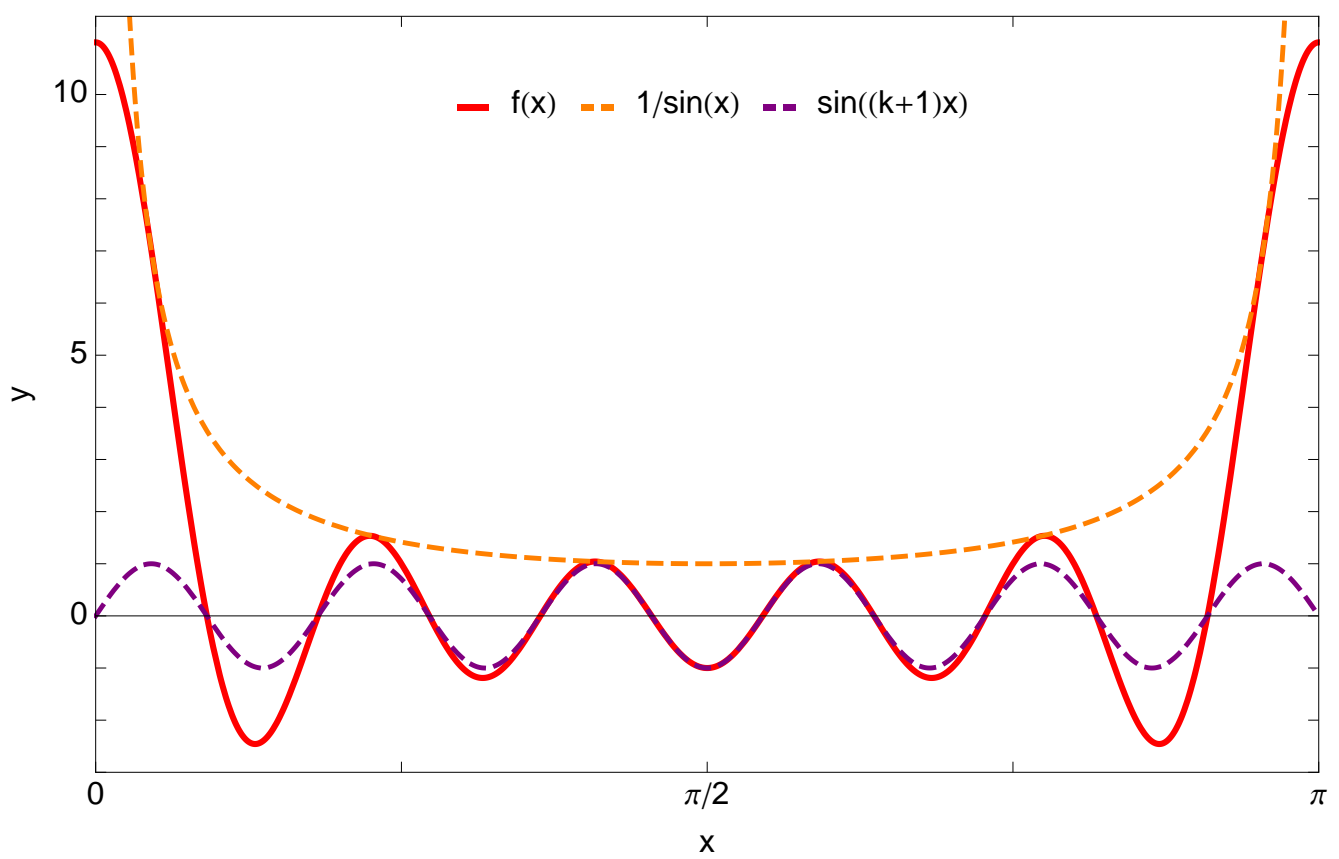

Figure 4.4: $\tilde{\lambda}_{2}^{\mathrm{mf}}\left(=\tilde{\lambda}_{N}^{\mathrm{mf}}\right.$ by symmetry $)$ always constitutes the second largest eigenvalue. Functions $f(x)$ (4.3.3), the oscillating function $\sin ((k+1) x)$ and the envelope function $1 / \sin (x)$ are plotted with $x=\frac{(l-1) \pi}{N} \in(0, \pi)$ for $k=10$. Obviously, a larger $k$ leads to more roots of $f(x)$, but otherwise functions show the same characteristics for all $k \leq N-1: f(x)$ has a local maximum at $x=0$ and decreases strictly monotonically up to the following minimum. For larger $x$ the envelope function guarantees that all values up to $x=\pi / 2$ are smaller than $f\left(x_{l=2}=\frac{\pi}{N}\right)$.

and $x \in(0, \pi / 2)$. Due to the symmetry (4.3.1) the interval $(0, \pi / 2)$ covers the entire spectrum (4.2.29). The function $f(x)$ on $x \in(0, \pi / 2)$ is the product of the oscillating function $\sin ((k+1) x)$ and a strictly monotonically decreasing function $1 / \sin (x)$. Therefore, it is a damped oscillation with period of $2 \pi /(k+1)$ and with the amplitude decreasing as $1 / \sin (x)$ (Fig. 4.4).

At $x=0$ we apply the Theorem of l'Hospital to calculate the following limits. There is a removable singularity

$$
\lim _{x \rightarrow 0} f(x)=k+1
$$

with

$$
\lim _{x \rightarrow 0} f^{\prime}(x)=0 \text { and } \lim _{x \rightarrow 0} f^{\prime \prime}(x)=-\frac{1}{3} k\left(k^{2}+3 k+2\right)<0,
$$

i.e. a local maximum.

In order to show that the index $l=2$ is always associated with the second largest 


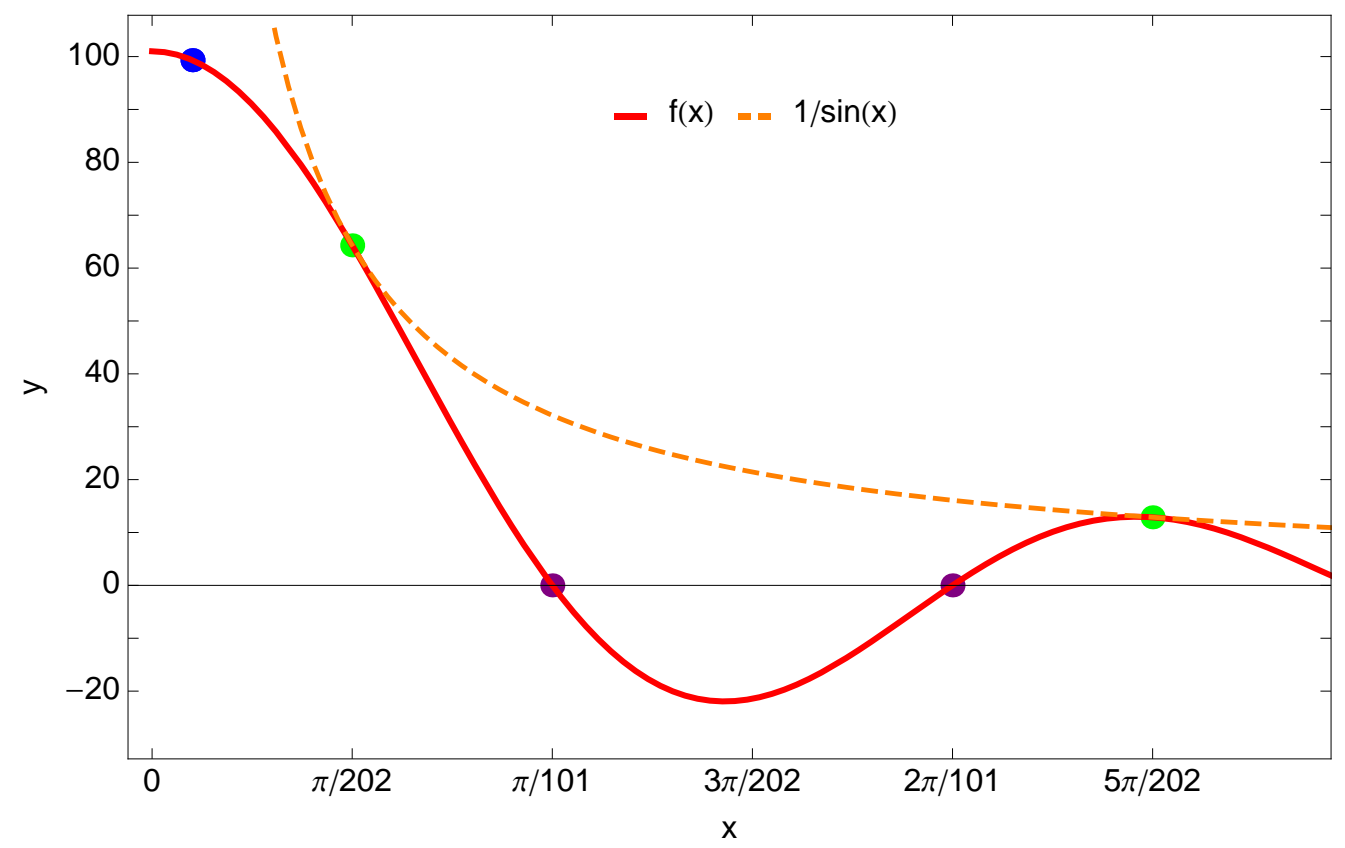

Figure 4.5: Important points of the function $f(x)$. The function $f(x)$ is plotted for $k=100$. The boundary points (green, eq. (4.3.9)) of the function $f(x)$ and the envelope function $1 / \sin (x)$, roots of $f(x)$ (purple, eq. (4.3.8) ) and the $\mathrm{x}$-value $x_{l=2}$ (blue, eq. (4.3.8), $N=1000$ ) corresponding to the eigenvalue $\lambda_{2}$ are highlighted.

eigenvalue, we first determine its $x$-value. It is given by

$$
x_{l=2}=\frac{\pi}{N} .
$$

Since the roots of the function $f(x)$ are located at

$$
x_{\text {root }, r}=\frac{r \pi}{k+1}
$$

for $r \in \mathbb{Z}$. Thus, $x_{l=2}$ is always smaller than the first root $x_{\text {root }, 1}$ (4.3.8) of the function $f(x)$ (Fig. 4.5) .

The boundary points of function $f(x)$ and the envelope function $1 / \sin (x)$ are given by

$$
x_{b, r}=\frac{4 \pi r+\pi}{2(k+1)} .
$$

for $r \in \mathbb{Z}$.

The function $f(x)$ is bounded from above by the envelope function $1 / \sin (x)$ for all $x>x_{b, 0}$ with

$$
x_{b, 0}=\frac{\pi}{2(k+1)}
$$

being the first boundary point of function $f(x)$ and its envelope function $(r=0$ in eq. (4.3.9) ) (Fig. 4.5) . 
The first derivative of $f(x)$ stays negative at least up to the first root $(r=1$ in eq. (4.3.8)) at

$$
x_{\text {root }, 1}=\frac{\pi}{(k+1)} .
$$

The first root $x_{\text {root }, 1}$ is always larger than the first boundary point $x_{b, 0}$ (4.3.10).

To summarize, the function $f(x)$ has a local maximum at $x=0$ and is then strictly monotonically decreasing up to $x_{b, 0}$ (4.3.10). Then, for all $x>x_{b, 0}$ the function $f(x)$ takes values smaller than or at most equal to the values of the envelope function $1 / \sin (x)$, which is strictly monotonically decreasing in the considered domain (see Figures ?? and 4.5).

Thus, if the $x_{l=2}$ (4.3.7) is smaller than the first boundary point $x_{b, 0}$ (4.3.10), the eigenvalue $\lambda_{2}$ constitutes indeed the second largest eigenvalue.

Comparing equations (4.3.7) and (4.3.10), this is the case for $N \geq 2(k+1)$, i.e. for $k<N / 2$.

Numerical investigations suggest that the eigenvalue $\lambda_{2}$ always constitutes the second largest eigenvalue independent from the chosen values for the parameters $N$, $k$ and $q$. However, monotonicity considerations are not that evident for $k>N / 2$.

The other extremal eigenvalue $\tilde{\lambda}_{\text {min }}^{\text {mf }}$ can not be that easily assigned to a fixed index. It is possible to find good estimates for the index at which the smallest eigenvalue $\tilde{\lambda}_{\min }^{\mathrm{mf}}$ always occurs. However, for the purposes of this thesis we simply define it as

$$
\tilde{\lambda}_{\min }^{\mathrm{mf}}=\min _{l} \tilde{\lambda}_{l}^{\mathrm{mf}} .
$$

\subsection{Extreme eigenvalues}

As the offset of each eigenvalue (4.2.29) equals $k$, we consider the scaled eigenvalues

$$
\lambda_{l}^{\mathrm{mf}}(N, k, q)=\frac{\tilde{\lambda}_{l}^{\mathrm{mf}}(N, k, q)}{k}
$$

in the following to allow for a consistent analysis for different $k$.

We first focus on the second largest eigenvalue since long time dynamics are dominated by it (see Chapter 3). For $l=2$, eq. (4.2.29) simplifies to

$$
\begin{aligned}
\lambda_{2}^{\mathrm{mf}}(N, k, q)= & -1+c^{\prime}\left(\frac{\sin \left(\frac{(k+1) \pi}{N}\right)}{\sin \left(\frac{\pi}{N}\right)}-1\right) \\
& +c^{\prime \prime}\left(\frac{\sin \left(\frac{(k+1) \pi}{N}\right)}{\sin \left(\frac{\pi}{N}\right)}\right) .
\end{aligned}
$$

The analytic prediction (4.4.2) well fits with the typical eigenvalues of actual small-world networks obtained by numerical diagonalization, cf. Fig. 4.6, It turns out that the analytic prediction is accurate for both undirected and directed networks, and for all but very small relative degrees $k / N<0.05$. For small $k$, the prediction still is a good guide for the general dependence of the second largest 


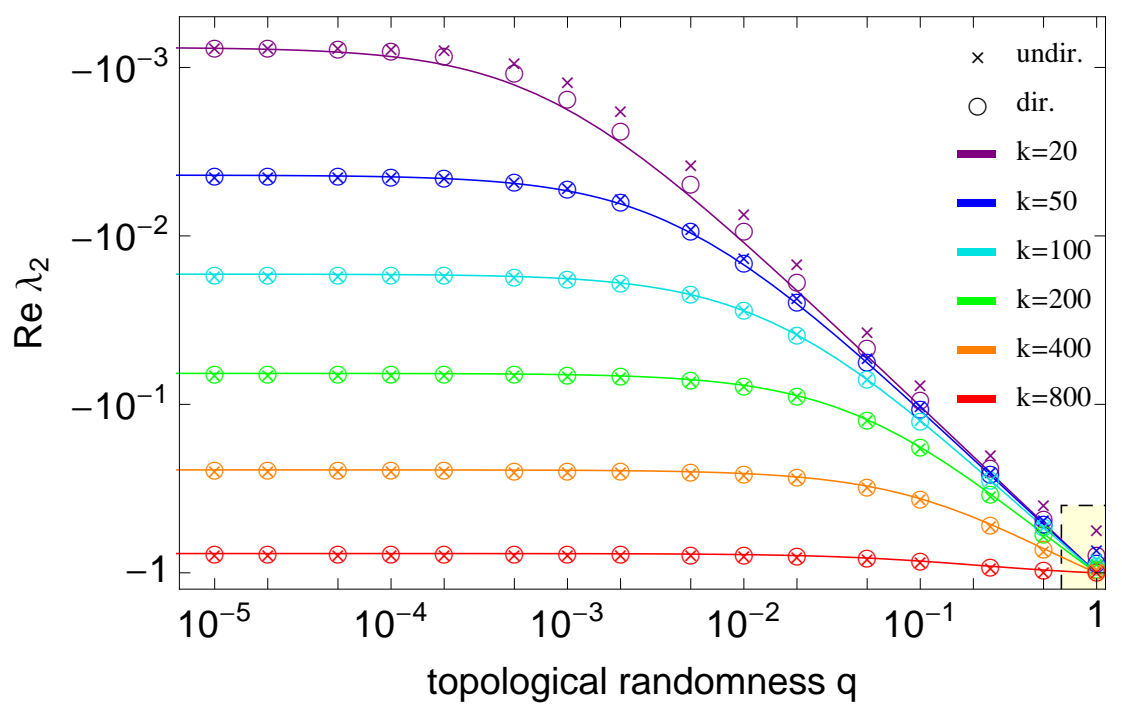

Figure 4.6: Accuracy of analytic prediction of the second largest eigenvalues from regular to randomized networks. Numerical measurements for undirected $(\times)$ and directed $(\bigcirc)$ networks in comparison with the analytical mean field predictions (Eq. (4.4.2), solid lines) as a function of $q$, for different degrees $k$. The error bars on the numerical measurements are smaller than the data points $(N=1000$, each data point averaged over 100 realizations).

eigenvalue on $q$, but shows some deviation from the numerical results. Moreover, the prediction (4.4.2) well approximates the actual dependence of $\lambda_{2}$ for all but very large $q$, thus including regular rings, small-world and even more substantially randomized network topologies.

\subsubsection{Approximation for small degrees}

Expanding eq. (4.4.2) up to $O\left(N^{2}\right)$ as $N \rightarrow \infty$ yields

$$
\begin{aligned}
\lambda_{2}^{\mathrm{mf}}(N, k, q) \simeq & -q-\frac{(1+k(1-q)) q}{N} \\
& -\frac{(k+1)(k+2) \pi^{2}(1-q)+6 q(k+1-k q)^{2}}{6 N^{2}} .
\end{aligned}
$$

For $q=0$ we recover the known approximation for symmetric regular ring networks

$$
\lambda_{2}^{\mathrm{mf}}(N, k, 0) \simeq-\frac{(k+1)(k+2) \pi^{2}}{6 N^{2}} .
$$

The approximation (4.4.4) well agrees with eq. (4.4.2) up to values of $k<N / 2$, but still is a good guide for even larger degrees $k$, cf. Fig. 4.7. 


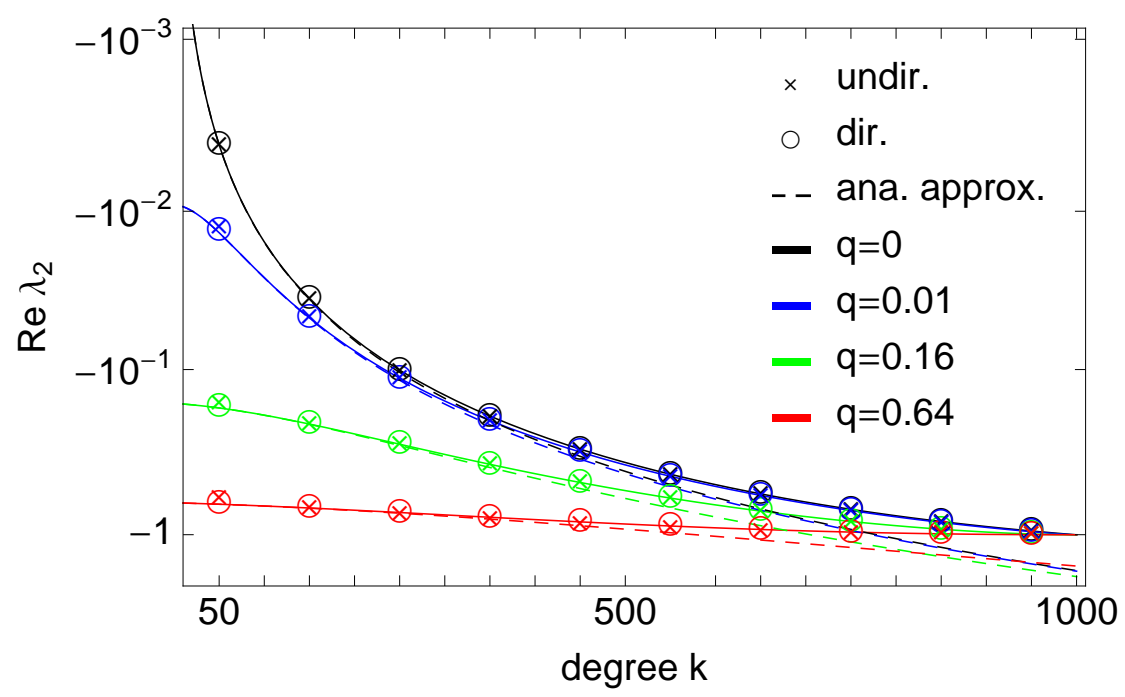

Figure 4.7: Scaling of the real part of the second largest eigenvalue for fixed network size. The analytical approximations obtained from the expansions in eq. (4.4.4) are depicted by the dashed lines. The error bars on the numerical measurements are smaller than the data points, $N$ fixed to 1000 .

\subsubsection{Scaling with network size}

How does the second largest eigenvalue depend on the network size? Fixing the edge density $d=k / N$ for large $N \gg 1$ ensures that networks stay connected and yields the prediction

$$
\lambda_{2}^{\mathrm{mf}}(d, q) \simeq-1+\frac{(1-d)(1-q)}{(1-d(1-q)) d \pi} \sin (d \pi)
$$

in the limit $N \rightarrow \infty$.

Our analytic prediction (4.4.6) again well approximates the real part of the second largest eigenvalue in dependence on the edge density $d$ for networks of size above about $N=500$ nodes, for both undirected and directed networks, cf. Fig. 4.8, For edge densities other than that displayed $(d=k / N=0.1$ and $d=0.5$, Fig. 4.8 (b),(c)) the real parts of the second largest eigenvalue show qualitatively the same asymptotic behaviour.

\subsubsection{The smallest eigenvalue}

The smallest eigenvalue $\lambda_{\min }$ defined in eq. (4.3.12) is also an important indicator for synchronization properties, in particular for the synchronizability (see e.g. [BP02]).

Here, the analytic prediction (4.4.2) again well fits with the actual eigenvalues obtained by numerical diagonalization, cf. Fig. 4.9. It turns out that the analytic prediction is accurate for both undirected and directed networks. The prediction (4.4.2) well approximates the actual dependence of $\lambda_{\min }$ for small $q$, thus including 

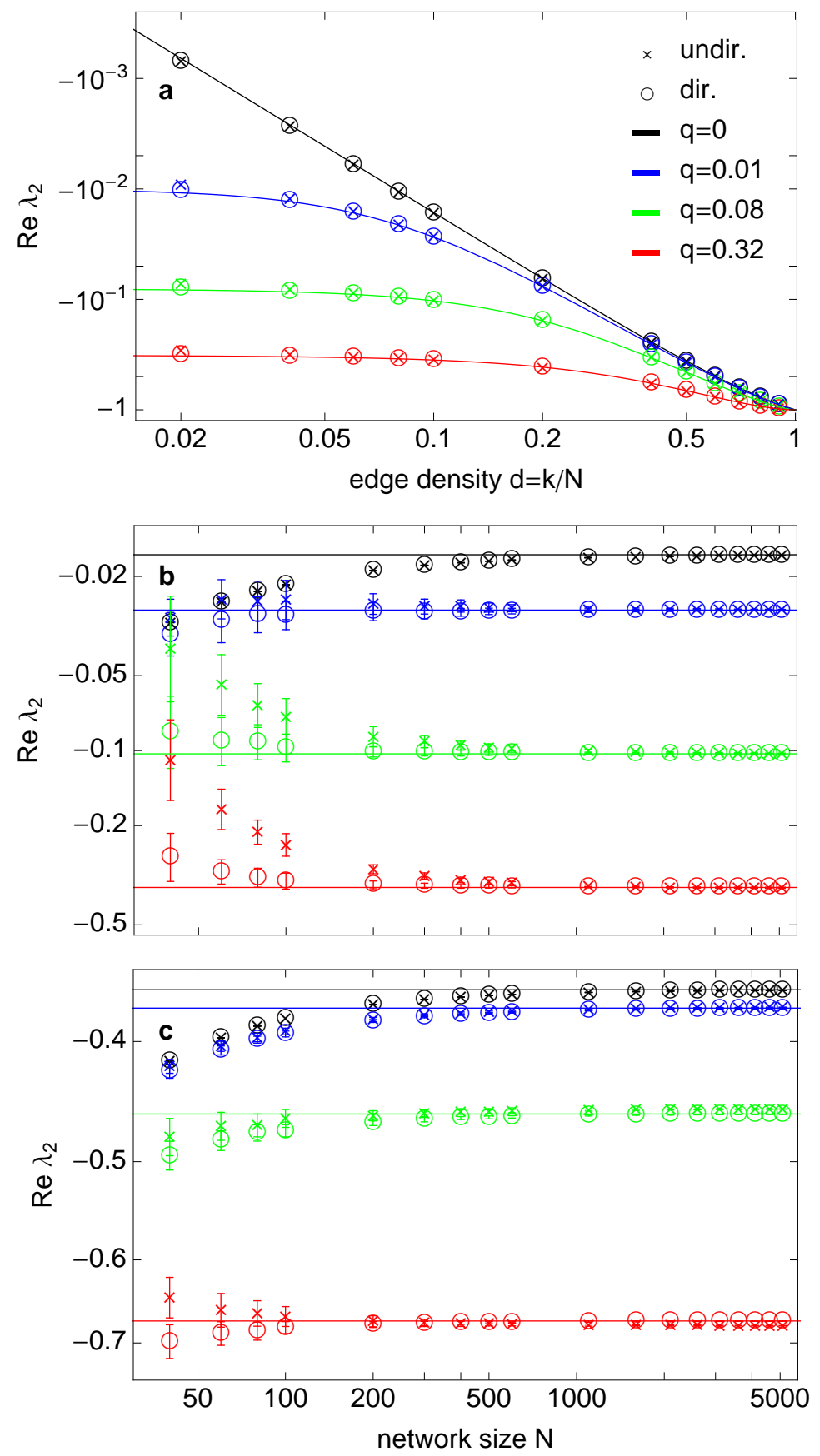

Figure 4.8: Second largest eigenvalues in dependence on edge density and network size. a: Numerical measurements for directed $(\bigcirc)$ and undirected $(x)$ networks (error bars smaller than the data points) in comparison with the analytic mean field prediction (4.4.6) for $N=2000$. b: Asymptotic $(N \rightarrow \infty)$ real parts of the second largest eigenvalues $\lambda_{2}$ in dependence on the network size $N$ for fixed edge density $d=k / N=0.1$ $(q$-values and symbols as in $(\mathbf{a}))$. c: Asymptotic $(N \rightarrow \infty)$ real parts of the second largest eigenvalues $\lambda_{2}$ in dependence on the network size $N$ for fixed edge density $d=k / N=0.5$ ( $q$-values and symbols as in (a)). 


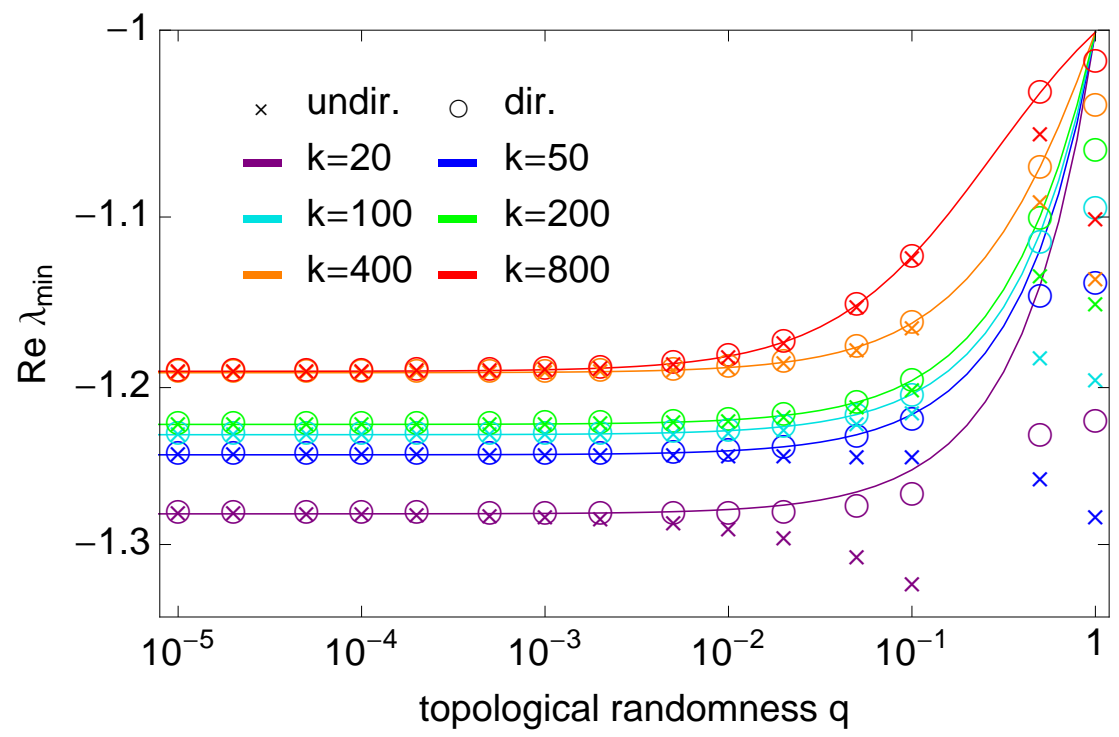

Figure 4.9: Accuracy of analytic prediction of smallest eigenvalues from regular to randomized networks. Numerical measurements for undirected $(x)$ and directed $(\bigcirc)$ networks in comparison with the analytical mean field predictions (Eq. (4.4.2), solid lines) as a function of $q$, for different degrees $k$. The error bars on the numerical measurements are smaller than the data points $(N=1000$, each data point averaged over 100 realizations).

regular rings and small worlds. The prediction is still a good guide for the general dependence of the second largest eigenvalue on $q$, but shows some deviation from the numerical results for larger $q$, i.e. for substantially randomized network topologies.

\subsection{Analytical predictions for random topologies via Random Matrix Theory}

To analytically predict the second largest eigenvalues for the graph Laplacians of undirected and directed networks close to $q=1$ (see the shaded area in Fig. 4.6) we consult Random Matrix Theory [Wig51] (cf. also [Por65, Meh91, TV09, ER05]). For a review of synchronization in networks with random interactions cf. [FJD06].

Firstly, we consider the undirected networks associated with symmetric matrices. Here, every connection between a pair of nodes $i$ and $j \neq i$ is present with a given probability $P$.

Secondly, we consider the directed networks associated with asymmetric matrices. Here, all nodes have the same in-degree $k_{i}^{i n}=k^{i n}$. Each of the $k^{i n}$ nodes that is connected to node $i$ is independently drawn from the set of all other nodes in the network with uniform probability.

Given a sufficiently large network size $N$ and a sufficiently large $k$ (respectively, 
a sufficiently large $k^{i n}$ ) we numerically find that the set of non-trivially eigenvalues resemble disks of radii $r^{\prime}$ for undirected networks and $r$ for directed networks (cf. also [TWG04, TGW06]).

Recall from eq. (4.1.1) that the graph Laplacian is obtained by shifting all eigenvalues of the adjacency matrix by $-k$. At least, this is exact for directed networks where the in-degree $k_{i}^{i n}=k$ for all nodes $i$ stays fixed during the whole rewiring procedure. For undirected networks there are small deviations from node to node but the average degree equals $k$. However, numerical simulations confirm that shifting here again the eigenvalues of the symmetric adjacency matrix by the negative average degree $-k$ is feasible. Thus, we consider the adjacency matrices in the following, $A^{\text {sym }}$ for undirected and $A^{\text {asym }}$ for directed networks and later shift them by $-k$.

\subsubsection{Ensembles of symmetric and asymmetric random matrices}

First, consider $N \times N$ symmetric matrices $A=A^{\top}$ with real elements $A_{i j}$. We constrain the diagonal entries to vanish $A_{i i}=0$ and we denote its $N$ eigenvalues by $\lambda_{k}$. The elements $A_{i j}(i<j)$ are independent, identically distributed random variables according to a probability distribution $\rho\left(A_{i j}\right)$. Due to [MF91, FM91, SC02 there is only one non-trivial ensemble with independent identically distributed matrix elements that differs from the Gaussian one, the ensemble of sparse random matrices is the only non-trivial ensemble with independent identically distributed matrix elements that differs from the Gaussian one. Thus there are exactly two universality classes, i.e. classes which do not depend on the probability distribution $\rho\left(A_{i j}\right)$, but are determined by matrix symmetry only. Every ensemble of matrices within one of these universality classes exhibits the same distribution of eigenvalues in the limit of large matrices, $N \rightarrow \infty$, but the eigenvalue distributions are in general different for the two classes. To obtain symmetric matrices, one chooses $A_{i j}=A_{j i}$ and $A_{i i}=0$ for both ensembles. Thus the arithmetic mean of the eigenvalues is zero,

$$
\left[\lambda_{i}\right]_{i}:=\frac{1}{N} \sum_{i=1}^{N} \lambda_{i}=\frac{1}{N} \sum_{i=1}^{N} A_{i i}=0
$$

and the ensemble variance of the matrix elements scale like

$$
\sigma^{2}=\left\langle A_{i j}^{2}\right\rangle \doteq \frac{r^{2}}{N}
$$

for $N \gg 1$.

For the Gaussian symmetric ensemble, it is known [Wig51, Meh91] that the distribution of eigenvalues $\rho_{\text {Gauss }}^{\text {sym }}(\lambda)$ in the limit $N \rightarrow \infty$ is given by Wigner's semicircle law

$$
\rho_{\text {Gauss }}^{\text {sym }}(\lambda)= \begin{cases}\frac{1}{2 \pi r^{2}} \sqrt{4 r^{2}-\lambda^{2}} & \text { if }|\lambda| \leq 2 r \\ 0 & \text { otherwise. }\end{cases}
$$

The ensemble of sparse matrices [BR88, Rod88, FM91, MF91, RAKK05, GT10] exhibits a different eigenvalue distribution $\rho_{\text {sparse }}^{\text {sym }}(\lambda)$ that depends on the finite number 
$k$ of nonzero entries per row and approaches the distribution $\rho_{\text {Gauss }}^{\text {sym }}(\lambda)$ in the limit of large $k$ such that

$$
\lim _{k \rightarrow \infty} \rho_{\text {sparse }}^{\text {sym }}(\lambda)=\rho_{\text {Gauss }}^{\text {sym }}(\lambda) .
$$

It is important to note that in the limit of large $N$ the distributions $\rho_{\text {sparse }}^{\text {sym }}$ and $\rho_{\text {Gauss }}^{\text {sym }}$ eigenvalues depend only on the one parameter $r$, that is derived from the variance of the matrix elements (4.5.2).

For real, asymmetric matrices (independent $A_{i j}$ and $A_{j i}$ ), there are no analytical results for the case of sparse matrices but only for the case of Gaussian random matrices. The Gaussian asymmetric ensemble yields the distribution of complex eigenvalues in a disk in the complex plane Gir85, SCSS88]

$$
\rho_{\text {Gauss }}^{\text {asym }}(\lambda)= \begin{cases}\frac{1}{\pi r^{2}} & \text { if }|\lambda| \leq r \\ 0 & \text { otherwise }\end{cases}
$$

where $r$ from Eq. (4.5.2) is the radius of the disk that is centered around the origin. Like in the case of symmetric matrices, this distribution also depends only on one parameter $r$, that is derived from the variance of the matrix elements.

\subsubsection{Undirected random networks}

The real symmetric adjacency matrix $A^{\mathrm{sym}}$ is an $N \times N$ matrix that satisfies $A_{i j}^{\mathrm{sym}}=$ $A_{j i}^{\mathrm{sym}}$ and $A_{i i}^{\mathrm{sym}}=0$.

Furthermore, the matrix elements of $A^{\mathrm{sym}}$ are independent up to the symmetry constraint $A_{i j}^{\mathrm{sym}}=A_{j i}^{\mathrm{sym}}$. They are equal to 1 with probability

$$
P=\frac{\left\langle k_{i}\right\rangle}{N-1} \approx \frac{k}{N}
$$

and equal to 0 with probability $1-P$.

Thus, the variance $\sigma^{2}$ is given by

$$
\sigma^{2}=P(1-P)=\frac{k}{N}\left(1-\frac{k}{N}\right) .
$$

Therefore, the eigenvalues are located in a disc of radius

$$
r^{\prime}=2 r
$$

with

$$
\begin{aligned}
r & =\sigma \sqrt{N} \\
& =\sqrt{\frac{k}{N}\left(1-\frac{k}{N}\right)} \sqrt{N} \\
& =\sqrt{k-\frac{k^{2}}{N}}
\end{aligned}
$$

centered around the origin. 


\subsubsection{Directed random networks}

The real asymmetric adjacency matrix $A^{\text {asym }}$ has exactly $k$ elements equal to one per row. Therefore, its elements have an average

$$
\left[A_{i j}^{\mathrm{asym}}\right]=\frac{1}{N} \sum_{j=1}^{N} A_{i j}^{\mathrm{asym}}=\frac{k}{N}
$$

and a second moment

$$
\left[A_{i j}^{\mathrm{asym} 2}\right]=\frac{1}{N} \sum_{j=1}^{N} A_{i j}^{\mathrm{asym} 2}=\frac{k}{N}
$$

Thus, the variance

$$
\sigma^{2}=\left[A_{i j}^{\text {asym } 2}\right]-\left[A_{i j}^{\text {asym }}\right]^{2}
$$

is given by

$$
\sigma^{2}=\frac{k}{N}-\frac{k^{2}}{N^{2}}
$$

If we assume that the eigenvalue distribution for directed networks with fixed in-degree is similar to those for random matrices [TWG04, TGW06, we obtain a prediction

$$
r^{2} \approx N \sigma^{2}
$$

from Eq. (4.5.2), which yields

$$
\begin{aligned}
r & \approx \sqrt{\frac{k}{N}-\frac{k^{2}}{N^{2}}} \sqrt{N} \\
& =\sqrt{k-\frac{k^{2}}{N}}
\end{aligned}
$$

for the radius of the disk of eigenvalues centered around the origin.

\subsubsection{Predictions for the scaled graph Laplacians}

To obtain predictions for the eigenvalues of the appropriate graph Laplacian we have to consider the shift by $-k$, discussed in the beginning of this section, and the scaling factor $1 / k$ introduced in eq. (4.4.1).

With it and eq. (4.5.11), the second largest eigenvalues for undirected networks close to $q=1$ (Fig. 4.10, (a)) are well predicted by

$$
\begin{aligned}
\lambda_{2}^{\mathrm{wsc}}(N, k, 1) & =\frac{1}{k}\left(2 \sqrt{k-\frac{k^{2}}{N}}-k\right) \\
& =2 \sqrt{\frac{1}{k}-\frac{1}{N}}-1,
\end{aligned}
$$




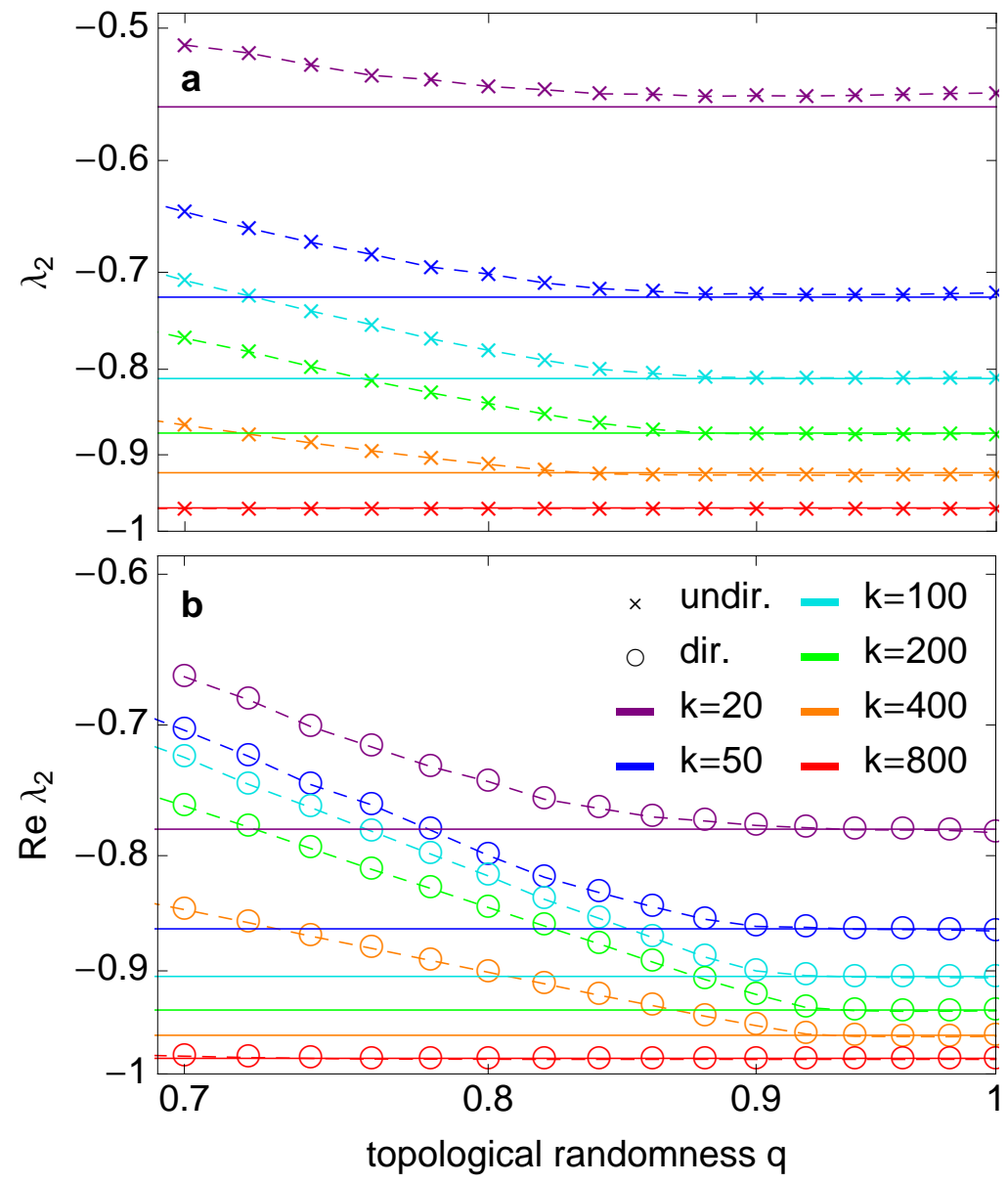

Figure 4.10: Accuracy of analytic prediction of the second largest eigenvalues close to $q=1$. a: Numerical measurements for undirected $(\times)$ networks in comparison with the analytical predictions $\lambda_{2}^{\text {wsc }}$ via Wigner's semi-circle law (Eq. (4.5.20), solid lines), for different degrees $k$. b: Numerical measurements for directed $(\bigcirc)$ networks in comparison with the analytical predictions $\lambda_{2}^{\mathrm{rmt}}$ from the theory of asymmetric random matrices (Eq. (4.5.22), solid lines). The error bars on the numerical measurements are smaller than the data points ( $N=1000$, each data point averaged over 100 realizations). Dashed lines are only a guide to the eye. 
the real parts of them for directed networks close to $q=1$ (Fig. 4.10, (b)) with eq. (4.5.18) by

$$
\begin{aligned}
\lambda_{2}^{\mathrm{rmt}}(N, k, 1) & =\frac{1}{k}\left(\sqrt{k-\frac{k^{2}}{N}}-k\right) \\
& =\sqrt{\frac{1}{k}-\frac{1}{N}}-1 .
\end{aligned}
$$

Note that $\lambda_{2}^{\text {wsc }}(N, k, 1)$ in eq. (4.5.20) acquires a positive value for too small $k$ values and a sufficiently large network size $N$ (cf. [FDBV01]). However, for the $k$-values we investigated (Fig. 4.10, (a) and (b)), the second largest eigenvalues are well predicted by both eqs. (4.5.20) and (4.5.22).

\subsection{The entire spectrum in mean field theory}

To gain further insights into the entire spectrum we study the density of states $\rho(\lambda)$ (cf. e.g. [FDBV01]) as defined in its discrete form, i.e. for finite network size $N$, by

$$
\rho(\lambda)=\frac{1}{N} \sum_{j=1}^{N} \delta\left(\lambda-\lambda_{j}\right),
$$

where $\delta$ is the Dirac delta function. The evaluation of (4.6.1) for the analytic mean field predictions and for the numerically obtained eigenvalues of undirected and directed networks are shows good qualitative agreement, cf. Fig. 4.11 for all but large topological randomness $q \rightarrow 1$. Spectra for networks with parameters other than $N=1000$ and $k=50$ yield qualitatively the same structure. Thus, the largest and smallest eigenvalues, the location and form of bulk peak as well as the entire structure of eigenvalues are well approximated up to $q$ of order 1 by the mean field predictions derived analytically.

\subsection{Summary and discussion}

In this Chapter we introduced a simple two-stage mean-field rewiring scheme which we used to derive analytical predictions for the spectra of graph Laplacians. Systematic numerical checks confirm that this prediction is accurate for the second largest eigenvalue for all but very small degrees or very large topological randomness. For very small $k$, our analytic prediction still serves as a valuable guide for the overall dependence all topological parameters. For $q$ close to unity, our mean field prediction can be complemented by existing results from random matrix theory. Besides the second largest and smallest eigenvalues, that already give valuable information about initial and asymptotic relaxation dynamics, the bulk spectrum as well as the fine structure of the spectrum is well approximated by our analytical prediction. In particular, the spectral prediction include regular rings, small worlds, and substantially more randomly rewired networks and undirected as well as directed ones. 

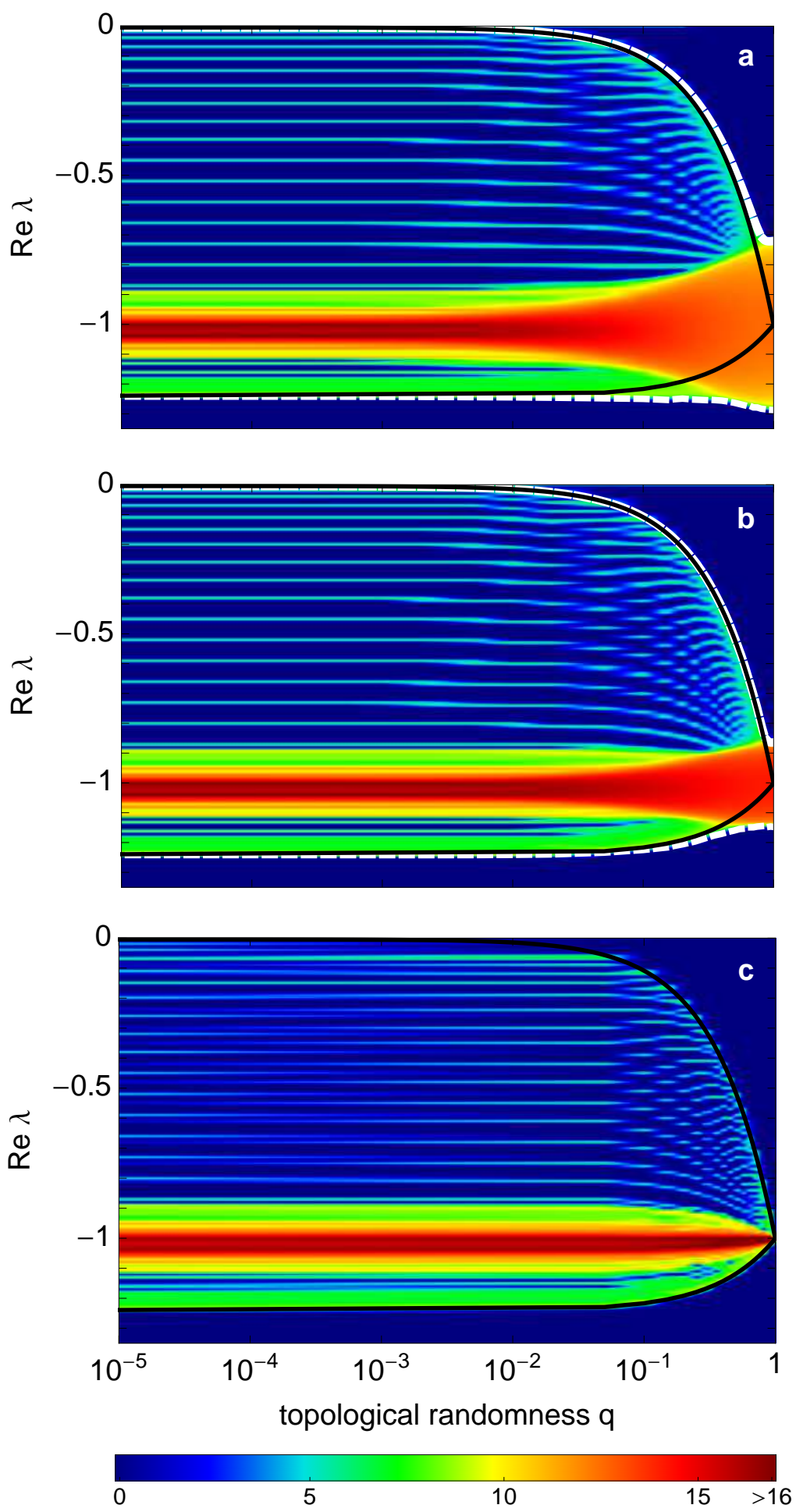

Figure 4.11: Analytics predicts structure of entire spectrum. Densities of states (eq. (4.6.1) for (a) undirected, (b) directed and (c) mean field networks $(N=1000, k=50)$. Dashed white lines show the extreme eigenvalues obtained numerically for undirected and directed networks. The solid black lines show the mean field prediction for the extreme eigenvalues. Densities of states for directed and undirected networks are averaged over 100 realizations for a fixed $q$-value, while the mean field density is analytically determined by eq. (4.2.29). Adapted from [GGT12. 
In particular, our theoretical predictions agree well with the eigenvalues obtained numerically over almost the entire range of topological randomness $q$, thereby completing previous attempts based on perturbation theory for $q \ll 1$ [Mon99, BP02].

Although the mean field rewiring is undirected, eigenvalues for directed networks are approximated more accurately and in a wider range of $q$-values, which is in particular related to the fact that the predictions for the undirected second-largest eigenvalues at $q=1$ are larger in real part than the directed ones, while all the mean field eigenvalues converge to -1 at $q=1$. For 'small' $k$-values the mean field approximation becomes less accurate, which may be due to the fact that the ring structure is destroyed more easily while rewiring. Additionally, the bulk spectra spread much more drastically with $q$ than for larger $k$-values.

The simple mean field approach presented here definitely leads to an essential reduction of computational efforts when studying randomized (regular or smallworld) network models. It may be extended to rewiring approaches starting from other than ring-like structures, for instance to two or three dimensions, as for instance, relevant for neural network modeling [SB09]. Checking with appropriate models, it may thus serve as a powerful tool to predict or deduce the relations between structural and dynamical properties of randomized networks.

Furthermore, the analysis of the mean field spectrum presented here could be extended to the Laplacian eigenvectors. Studies of the Laplacian eigenvectors are rare although there are fascinating results as well. For instance, the discrete analogs of solutions of the Schrödinger equation on manifolds can be investigated on graphs (cf. e.g. [BL07]). 



\section{Hubs Orchestrate Synchrony}

In this Chapter we present possible mechanisms determining how synchronous population activity in developing neuronal networks may be suppressed by targeted stimulation of so-called functional hubs based on recent experimental findings in neuroscience $\left[\mathrm{BGP}^{+} 09\right]$. We ask the reader to understand that we do not provide an introduction to theoretical neuroscience here. Instead, see e.g. Tuc88, KJJ96, DA01, GK02, Buz06. The work we present in this Chapter - unpublished of the time of writing - originates from a collaboration with Birgit Kriener (Institute of Mathematical Sciences and Technology, Norwegian University of Life Sciences, Ås, Norway) and Marc Timme.

Besides the small-world architecture, there is another network structure beyond the two extremes of totally regular and random ones, known as the scale-free topology [AB00, DM01, AB02, Cal07] since it is characterized by a heavy-tailed distribution of degree per neuron with no characteristic scale. While most neurons display local connectivity, there is a small number of hub neurons - characterized by the large numbers of cells they connect to - that have long-range connections. However, whether hubs are in fact present within neural assemblies has just been experimentally examined recently $\left[\mathrm{BGP}^{+} 09\right]$. Here, a morphological analysis revealed scale-free features in a functional topology of developing hippocampal networks. The high-connectivity (HC) neurons are a sub-population of $\gamma$-amino-butyric-acidreleasing (GABAergic) interneurons with widespread axonal arbors.

Intriguingly, stimulating a single $\mathrm{HC}$ or hub neuron completely suppresses global synchronous activity. When the stimulation is switched off, the synchronous activity returns. If a low-connectivity (LC) or non-hub neuron is driven in the same way, synchronous activity remains almost identically as without stimulation.

Such synchronous oscillations constitute one of the most dominant collective dynamics of complex networks. They occur not only in circuits neurons [BH99, Buz06, OLPT10], but in a large range of systems: ranging from metabolic and gene regulatory networks within cells [WF01, McM02, GdBLC03, TYHC03, to food webs of cross-feeding species [MHH98, WM00 or even to oscillations in the global climate system [SR94, SvRE98]. Thus, to understand the functional role of hubs - not only in neuronal circuits - is a question of paramount importance and has recently attracted attention [Per10, ZLZK10, SHK07, MS08, ASW ${ }^{+}$06, SR07]. However, the mechanisms underlying the suppression of global oscillations in a neurobiological system have not yet been understood. Understanding those mechanisms based on the phenomena observed in $\left[\mathrm{BGP}^{+} 09\right]$ is the main aspect we address in this Chapter. 


\subsection{Experimental findings}

Recently, experimental studies of the developing hippocampus of rat and mouse revealed that the functional connectivity - i.e. the connectivity defined in terms of correlations in the activity between neurons - follows a scale-free topology that includes the presence of functional hubs.

Using multibeam two-photon excitation of hippocampal slices together with fluorescent probes to study multineuron activity, Bonifazi et al. $\left[\mathrm{BGP}^{+} 09\right]$ determined that the connectivity within the networks was distributed as a power law, i.e. that the out-degree distribution is given by $P(k) \sim\left(k^{\text {out }}\right)^{-\alpha}$, with $\alpha \approx 1.1 \ldots 1.3$ (see Fig. 5.1).

First, single neurons were recorded using whole cell patch-clamp (current-clamp). They found that phasic stimulation of a small number of cells, which they designated hub neurons, induced rhythmic synapse-driven synchronizations, or giant depolarizing potentials (GDPs). Noting that these hub neurons are $\gamma$-aminobutyric acidĐreleasing (GABAergic) interneurons (INs) with long axonal arborizations, they next performed paired recordings where 'follower' neurons were recorded in voltage-clamp mode. These paired recordings revealed that hub neurons have lower action potential thresholds and receive more excitatory postsynaptic potentials (EPSPs). Finally, using cell-attached and whole-cell recordings, they present evidence that firing of hub neurons is indeed involved in spontaneous synchronization. Bonifazi et al. [BGP $\left.{ }^{+} 09\right]$ presume that two distinct classes of hub neurons, the first displaying long axons spanning regions with sparse collaterals, and the second showing a basket-like morphology with a dense but local arborization pattern, play a crucial role at early development stages, when GABA plays a complex role.

Furthermore, the neural circuits considered exhibit pronounced oscillations in their natural in vivo state that are to a very rough approximation periodic and exhibit a characteristic inter-peak time-interval (Fig 5.2).

Intriguingly, already externally driving single hub neurons (those with particularly large out-degree) may remove the collective oscillations completely (Fig 5.2). When the stimulation is switched off, they may emerge back. If a non-hub unit driven in the same way, the oscillation stays almost same as in the undriven state.

Thus the main overall question we want to address in the following is: What are the possible mechanisms removing the oscillations in the neurobiological system?

\subsection{The scale-free topology}

The neural circuit models we consider exhibit a power-law out-degree distribution $P(k) \sim\left(k^{\text {out }}\right)^{-\alpha}$. We abbreviate $k^{\text {out }}$ by $k$ in the following as long as this does not lead to any confusion. In [BGP $\left.{ }^{+} 09\right]$ it has been found that $\alpha$ equals approximately 1.3 (see Fig. 5.1)).

To create such a network we firstly generate, for each neuron independently, a random out-degree $k$ according to this power law and, secondly, randomly link uniformly to presynaptic neurons. For practical reasons, we create a continuous random number between two bounds, $x \in\left[k_{\min }, k_{\max }\right]$ and set $k=\operatorname{round}(x)$ thereafter. The 
A

1

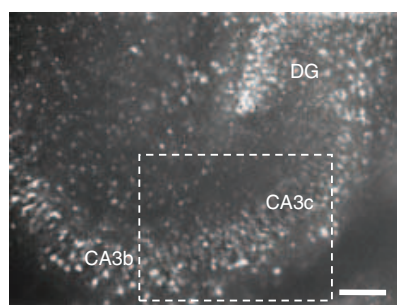

2

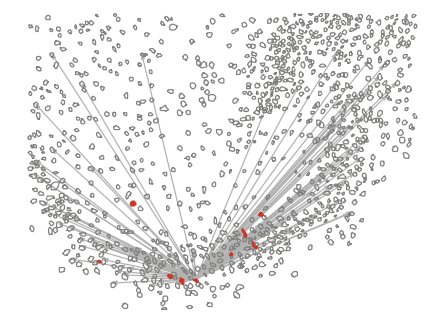

3

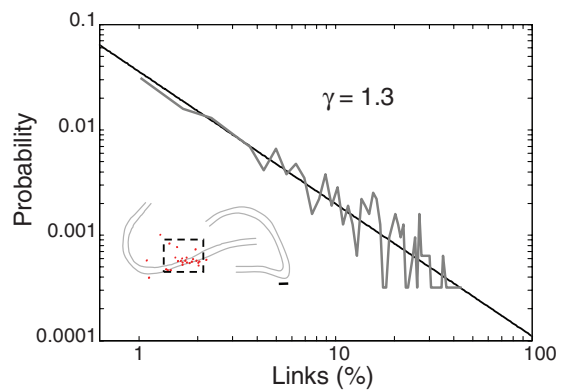

B

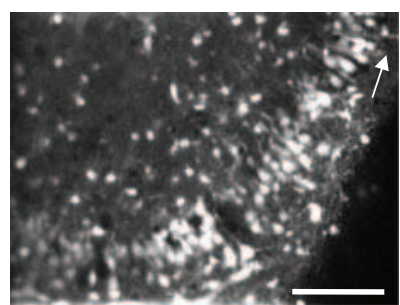

2

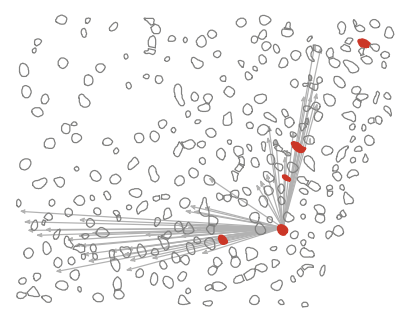

3

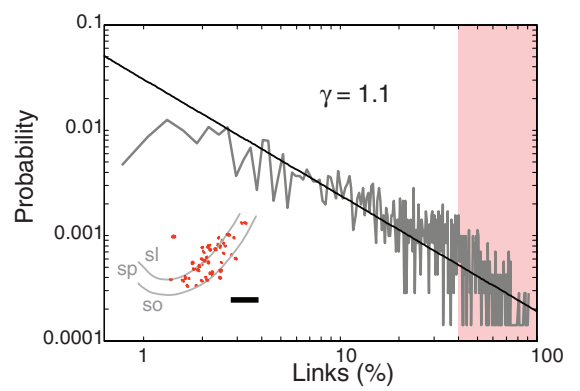

Figure 5.1: Analysis of multineuron calcium activity reveals a scale-free topology in the developing hippocampus. A: (1) Two-photon calcium fluorescence image of a rat hippocampal slice (scale bar: $100 \mu \mathrm{m}$. DG: dentate gyrus). (2) Detected contours of the cells from the fluorescence image shown in (1). Red dots are the 10 highest-connectivity neurons in the represented network; gray lines mark the out-degree links of one HC neuron. (3) Probability distribution plot of the fraction of out-degree links over the total population of active neurons (gray line, $N=3224$ neurons in four slices). A power-law distribution with a slope of $\gamma=1.3 \pm 0.1(n=4)$ is indicated in black. The inset shows the location of the 30 highest-connectivity neurons $(\approx 1 \%$ of the population, red dots) on a schematic representation of the hippocampus. B: Same as A, but at x20 magnification (scale bar: $100 \mu \mathrm{m}$, sl: stratum lucidum; sp: stratum pyramidale; so: stratum) and for a population of 7588 neurons; similar probability distribution of out-degree links $(\gamma=1.1 \pm 0.1, n=45)$. The probability threshold for HC neurons was fixed to $40 \%$ (red-shaded area). The inset indicates the location of $\mathrm{HC}$ neurons (red dots) on a schematic representation of the CA3b/c region of the hippocampus. Note that we use $\alpha$ instead of $\gamma$ in this thesis. Adapted from $\left[\mathrm{BGP}^{+} 09\right]$. 


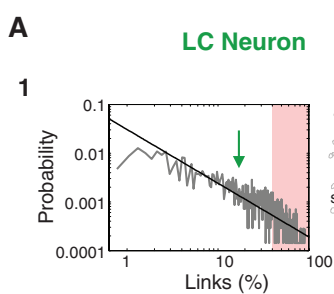

2

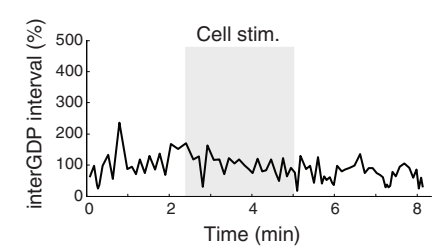

B

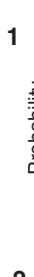

2

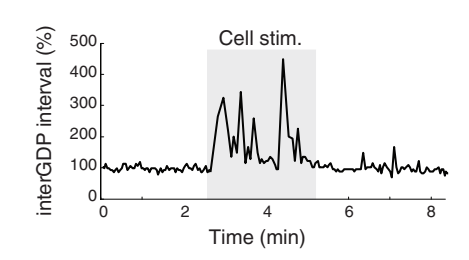

C

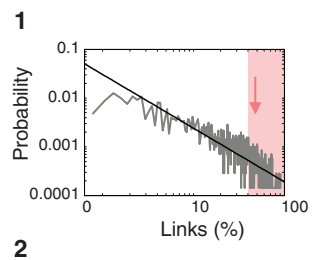

2

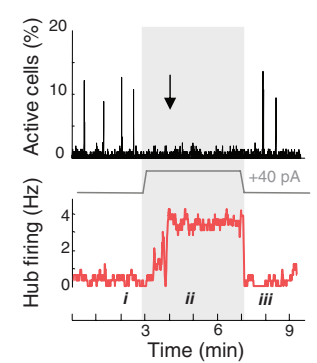

Figure 5.2: Stimulation of HC but not LC neurons affects network dynamics. A: Data from a representative LC interneuron. (1) The green arrow indicates the position in the pooled power-law distribution of out-degree links (Fig. 5.1, B) of the recorded neuron. Red-shaded area indicates the $\mathrm{HC}$ region. The right contour plot shows the position (solid red dot) and out-degree connections (gray lines) of the illustrated LC interneuron (scale bar, $100 \mu \mathrm{m}$ ). (2) Phasic current-clamp stimulation (200-ms pulses of 75-pA current every $10 \mathrm{~s}$, gray area) of the LC interneuron while being imaged did not affect the occurrence of GDPs (detected from the calcium activity). The interval between GDPs as a function of time is plotted. B: Same as $(\mathbf{A})$ but for a representative $\mathrm{HC}$ interneuron. Phasic stimulation of the HC interneuron [same protocol as $(\mathbf{A}, \mathbf{2})$ ] significantly decreased GDP frequency. C: Perturbations of network dynamics induced by the stimulation of $\mathrm{HC}$ interneurons. An HC interneuron preventing GDPs when stimulated. Graphs in (2) show the fraction of active cells (top histogram), as well as the cell firing frequency (bottom), as a function of time. Peaks of synchronous activity (GDPs) disappear when the membrane potential of the cell is depolarized by continuous positive current injection (40 pA). Current-clamp traces show the activity in the HC neuron in resting (i and iii) and stimulated (ii, gray) conditions. The black arrow indicates the time when a significant effect on network dynamics starts. Adapted and modified from $\left[\mathrm{BGP}^{+} 09\right]$.

bounds are of practical relevance since if $k$ is too small for one (or more) neurons, the network might not be strongly connected (see Chapter 2), and $k$ is unbounded, it could be larger than the actual network size $N$ considered. Given the density (or, experimentally, the histogram)

$$
\rho(x)=C x^{-\alpha} \quad \text { for } \quad x \in\left[k_{\min }, k_{\max }\right],
$$


the distribution function is given by

$$
\begin{aligned}
P(X \leq x)=F(x) & =\int_{k_{\min }}^{x} \rho\left(x^{\prime}\right) d x^{\prime} \\
& =\frac{C}{\alpha-1}\left[x^{\prime-\alpha+1}\right]_{x}^{k_{\min }},
\end{aligned}
$$

where

$$
\tilde{C}=\frac{1}{k_{\min }^{-(\alpha-1)}-k_{\max }^{-(\alpha-1)}}
$$

is fixed by the normalization condition $F\left(k_{\max }\right)=1$. The inverse $F^{-1}$ of $F$ is given by

$$
F^{-1}(u)=\left(k_{\min }^{-(\alpha-1)}-\frac{u}{\tilde{C}}\right)^{-\frac{1}{\alpha-1}} .
$$

Now drawing a random number $U$ from the uniform distribution on $[0,1]$ and applying the inverse distribution function results in a random variable $X=F^{-1}(U)$ that has the density $\rho$ according to eq. (5.2.1).

\subsection{Pulse-coupled oscillators revisited}

To get first insight into possible mechanisms we again consider the pulse-coupled oscillators introduced in Section 2.2.2,

We consider $N$ pulse-coupled oscillators (referred to as neurons in the following) with delay time $\Delta$ and coupled via the coupling matrix $J$ as defined in eq. (2.2.1) with $\sigma<0$, i.e. we consider purely inhibitory coupling and a homogeneous input is guaranteed by normalizing with the factor $1 / k_{i}^{\text {in }}$ (see eq. 3.1.3) .

Recall the rise function from (3.4.29) which mediates interactions

$$
U_{i}(\phi):=\frac{I_{i}}{\gamma}\left(1-e^{-\gamma \phi}\right)
$$

where $I_{i}$ is the temporally constant input current of neuron $i$ and $\gamma$ the dissipation of the system. So far we have only considered identical neurons, i.e. $I_{i}=I$ for all neurons $i$. In this case for identical local units and homogeneous total input coupling strengths, the fully synchronous state $\phi_{i}(t)=\phi_{0}(t)$ with the collective period (3.4.18) exists (see also eq.(3.1.6)).

If we now introduce non-homogeneous driving,

$$
I_{i}=I+\Delta I_{i}
$$

and we change (one or more of) the $\Delta I_{i}$ smoothly from zero, the synchronous state is continuously transformed into a close-to-synchronous one, with the phase dynamics $\phi_{i}(t)$ smoothly deviating (with parameters $\left.\Delta I_{i}\right)$ from the synchronous one $\phi_{0}(t)$.

Given sufficiently small driving inhomogeneities $\Delta I_{i}$ such a close-to-synchronous periodic orbit (CSPO) is guaranteed to exist [ $\left.\mathrm{DTD}^{+} 04\right]$. Here, gradually introducing inhomogeneities to an input-homogeneous system that exhibits a completely 

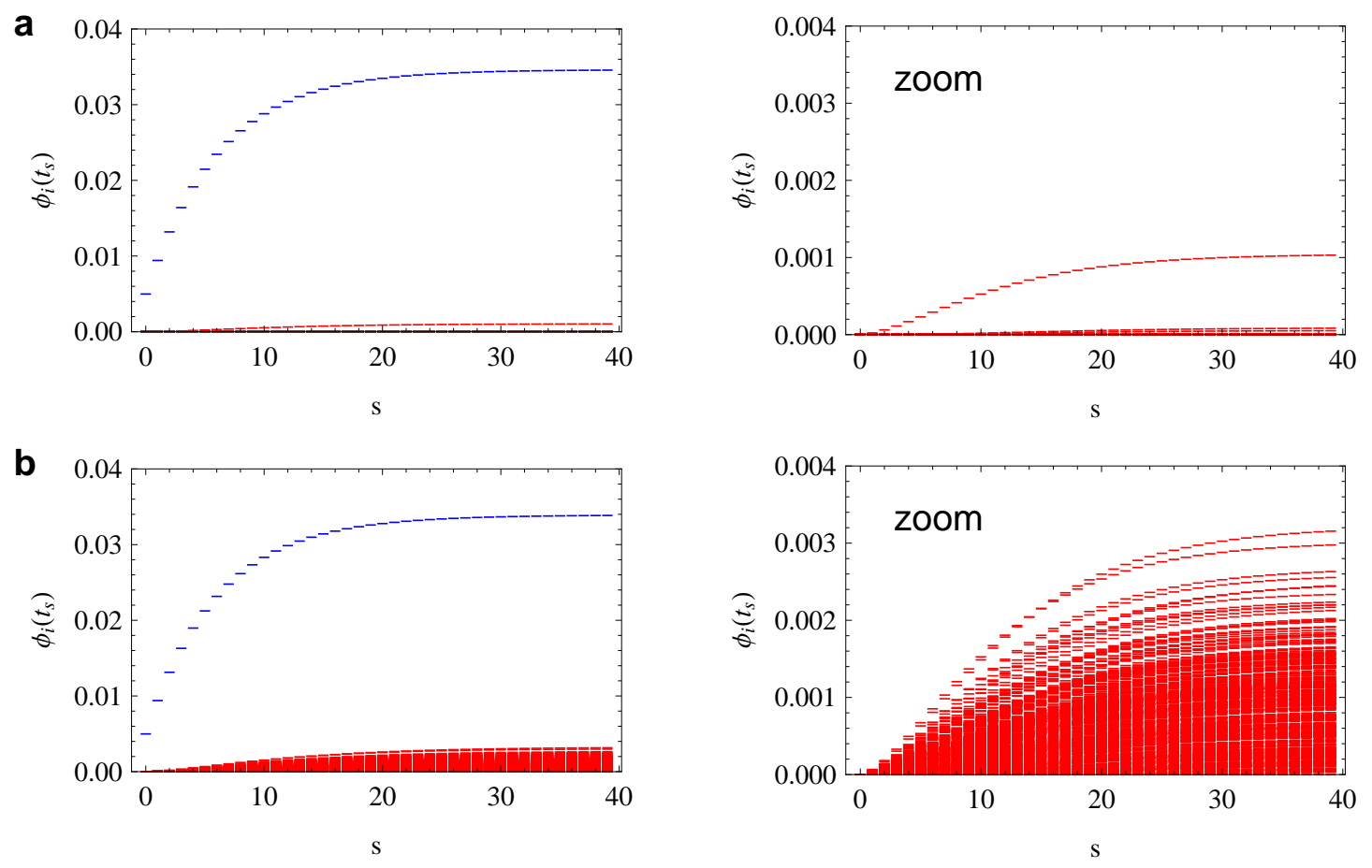

Figure 5.3: Phase spreads induced by driving a hub and a low-connected pulse-coupled oscillator in a network of 500 oscillators. Phases of all oscillators are plotted whenever a reference oscillator has been reset, i.e. at times $t_{s}$. The axis $s$ is thus discrete and nonlinear. Top row (a): The driven LC neuron (blue) reacts the strongest. Bottom row (b): The driven $\mathrm{HC}$ neuron (blue) reacts again the strongest with the induced phase shift being approximately the same. (Parameters: $N=500, \gamma=0.8, \sigma=-0.2$ ).

synchronous, periodic orbit leads to a split of phases close to synchrony. When this phase split becomes too large (of the order of the delay $\Delta$ ), the CSPO breaks down. In more detail, the CSPO ceases to exist if the heterogeneity is sufficiently large such that at least one neuron $i$ (out of all $\mathrm{N}$ neurons) receives at least one spike (out of all spikes from its postsynaptic neurons) before it actually spikes itself. In other words, the CSPO breaks down if the conditions

$$
\phi_{j}-\phi_{i}<\Delta
$$

for all neurons $j$ connected to neuron $i$ are not satisfied for at least one $i$. Thus, a sufficient - but not necessary condition - for the CSPO to exist is $\phi_{j}-\phi_{i}<\Delta$ for all $i, j \in\{1, \ldots, N\}$.

Thus, for this theoretical setup, our main question about possible mechanisms that suppress oscillations translates to the question: What are possible mechanisms destroying a CSPO?

To get an intuition about how the driving signals induce phase shifts given a network topology, we perform simulations where only one driving signal $\Delta I_{i}$ in 

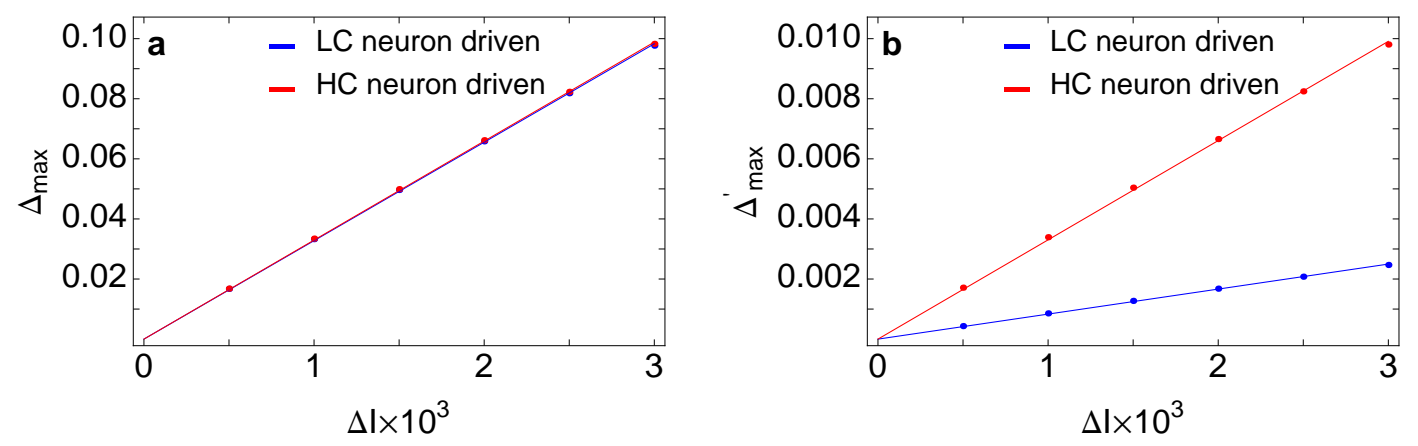

Figure 5.4: The maximal phase differences induced by driving a hub and a low-connected neuron. a: Considering the whole population of neurons, there is no significant difference between driving a $\mathrm{HC}$ or LC neuron (the driven neuron react the strongest (see Fig 5.3, blue lines)). The induced maximal phase difference is proportional to the driving signal $\Delta I . \mathbf{b}$ : Considering the population without the driven neuron there is a significant difference between driving a $\mathrm{HC}$ or $\mathrm{LC}$ neuron. Again the induced maximal phase difference is proportional to the driving signal $\Delta I$. (Parameters: $N=500$, leak $\gamma=0.8, \sigma=-0.2$ ).

eq. (5.3.2) is nonzero (only one neuron $i^{*}$ receives extra input).

Firstly, we create a scale-free topology as explained above. To get an impression whether driving a hub would have more impact on the phase spread than driving a non-hub, we choose the neuron with the minimal out-degree as the non-hub or LC neuron to be driven, the one with the largest out-degree as the hub or HC neuron to be driven.

In Fig. 5.3, the resulting phase spreads for both the LC and the $\mathrm{HC}$ neuron are depicted. The first observation is that the driven neuron react strongest, i.e. $\left|\Delta \phi_{i^{*}}\right|$ is largest. This agrees with results from [Tim07]. This $\left|\Delta \phi_{i^{*}}\right|$ is approximately of the same order for both the LC and the HC neuron. However, the impact on the neurons connected to the driven one (only one for the LC neuron in Fig. 5.3, b) are strongly unequal.

To quantify these effects we smoothly increase the induced inhomogeneities $\Delta I_{i^{*}}$ (Fig. 5.4). The induced maximal phase spreads increase proportionately to the input currents. With the phase spreads of the driven neurons being the maximal ones, there is no significant difference between driving the LC and the HC neuron (Fig. 5.4, a). However, excluding the driven neuron the stronger phase spread induced by the hub neuron gets apparent (Fig. [5.4, b).

Our simulations support the view that if only one unit is driven, the maximally possible phase split is achieved between the actually driven unit and some other unit. This supports the hypothesis that driving single units in a pulse-coupled system may induce similar effects to those obtained for continuous time phase- 
coupled oscillators [Tim07]. This work numerically suggests that if only one unit is driven, the maximally possible phase split is achieved between the actually driven unit and some other unit. Here, in almost the same manner, the driving neuron $i^{*}$ is the one that creates the transition to asynchrony, i.e. breaks one of the inequalities (5.3.3), either as $i^{*}=j$ or as $i^{*}=i$.

In order to be able to obtain analytical results, we will have to slightly modify the proposed framework. We may exclude the phase of the driven neuron from the phases considered for the determination of the maximal phase differences (see Fig. 5.4, b). Alternatively, we have to slightly modify the model, e.g. by incorporating a refractory period as in [RL11]. However, to figure out the decisive mechanisms relevant to destroying a CSPO (or underlying the suppression of global oscillations in the neurobiological system, respectively) we first numerically investigate biophysically more realistic neuron models.

\subsection{Biophysically more realistic neuron models}

To better understand the effects observed by $\left[\mathrm{BGP}^{+} 09\right]$ we now try a slightly more realistic modeling approach that takes into account temporally extended synaptic inputs modeled as conductances. Once having reproduced the phenomenon, we should then be able to reduce it to the building blocks necessary to uncover possible mechanisms that lead to a breakdown of the oscillations in the neurobiological system.

\subsubsection{Conductance-based leaky integrate-and-fire neurons}

First, we model the network as a population of conductance-based leaky integrateand-fire (cLIF) point neurons together with an excitatory background population modeled as Poisson point processes. Here, the first population represents GABAergic interneurons (IN), the second pyramidal neurons (pyr). The subthreshold membrane potential dynamics of each IN is given by

$$
\frac{d V(t)}{d t}=-\left(I_{\text {leak }}(t)+I_{\mathrm{syn}, \operatorname{exc}}(t)+I_{\mathrm{syn}, \mathrm{inh}}(t)\right) / C_{m}
$$

with membrane capacitance $C_{m}$ and excitatory (exc) and inhibitory (inh) synaptic input currents

$$
I_{\text {syn }, \text { exc } / \text { inh }}(t)=g_{\text {exc/inh }}(t)\left(V_{m}(t)-E_{\text {exc/inh }}\right)
$$

where

$$
g_{\text {syn,exc/inh }}(t)=\sum_{k} g_{0, \operatorname{exc} / \text { inh }} \frac{\left(t-t_{k}\right)}{\tau_{\text {syn }, \text { exc } / \text { inh }}} \mathrm{e}^{1-\left(t-t_{k}\right) / \tau_{\text {syn, exc } / \text { inh }}} H\left(t-t_{k}\right),
$$

are the synaptic conductances modeled as $\alpha$-functions with time constants $\tau_{\text {syn,exc/inh }}, t_{k}$ denotes the spike arrival times and $H(t)$ is the Heaviside step function. Finally,

$$
I_{\text {leak }}(t)=g_{\text {leak }}\left(V_{m}(t)-E_{\text {rest }}\right)
$$


is the leak current with leak conductance $g_{\text {leak }}$.

Whenever $V(t)$ reaches a threshold value $V_{\text {th }}$, the neuron emits a spike and $V\left(t^{+}\right)$ is reset to the resting potential $V_{\text {rest }}$ where it is clamped during a refractory time $\tau_{\text {ref }} . E_{\text {exc/inh }}$ are the reversal potentials of the excitatorily and inhibitorily acting ion channels, respectively, at which flux of ions reverses directionality. Hence, if $V(t)<E_{\text {inh }}$ the net inhibitory current actually acts depolarizingly on $V(t)$ (and vice versa for excitatory currents). In mature neurons this is rarely the case since $V(t)$ is usually above $E_{\mathrm{inh}} \sim-75 \mathrm{mV}$ which is of the order of the resting potential, and thus it is quite save to assume that inhibitory currents act hyperpolarizingly, i.e. driving $V(t)$ away from threshold. In immature developing hippocampal networks however $E_{\mathrm{inh}}=E_{\mathrm{GABA}} \in[-46,-36] \mathrm{mV}$ [BAGTK07] and "inhibitory" (GABA-mediated) currents can actually be depolarizing.

We moreover assume short-term plasticity of the IN synapses [TPM98] mimicking the limited resources of synaptic neurotransmitter and its reuptake:

$$
I_{\mathrm{syn}, i}(t)=\sum_{j} J_{i j} s_{\mathrm{act}, i j}(t)
$$

with $J_{i j}$ being the absolute coupling strength and the $s_{\text {act }, i j}(t)$ determined by

$$
\begin{aligned}
\frac{d s_{\text {rec }}(t)}{d t} & =\frac{s_{\text {inact }}}{\tau_{\text {rec }}}-u s_{\text {rec }} \delta\left(t-t_{k}\right) \\
\frac{d s_{\text {act }}(t)}{d t} & =-\frac{s_{\text {act }}}{\tau_{\text {syn }}}+u s_{\text {rec }} \delta\left(t-t_{k}\right) \\
\frac{d s_{\text {inact }}(t)}{d t} & =\frac{s_{\text {act }}}{\tau_{\text {syn }}}-\frac{s_{\text {inact }}}{\tau_{\text {rec }}},
\end{aligned}
$$

where $s_{\text {rec }}, s_{\text {act }}$ and $s_{\text {inact }}$ are the fractions of synaptic resources in recovered, active and inactive states, respectively. Furthermore, $\tau_{\text {syn }}$ is the time constant of the synaptic current, $\tau_{\text {rec }}$ the recovery time constant and $u$ is the effective use of synaptic resources, which increases with spikes if facilitation is included.

\subsubsection{Choice of parameters}

Parameters were taken - when possible - from [BGP $\left.{ }^{+} 09\right]$ and the review [BAGTK07], cf. Table (5.4.2). Synaptic short-term plasticity parameters were chosen as presented in Table (5.4.2). All simulations were performed with the NEST simulator GD07.

Fig. (5.5) shows $20 \mathrm{~s}$ of spiking and population activity of 1000 neurons under different stimulus conditions, cf. figure caption for details. The same direct current stimulus that was applied in the experiments $(40 \mathrm{pA})$ was applied to a subset of in this case 11 out of $27 \mathrm{HC}$ neurons from $t=4 \mathrm{~s}$ to $t=8 \mathrm{~s}$. The GDPs are suppressed. The same stimulus applied to a subset of 11 LC neurons does not suppress GDPs.

This reproduces some of the observations from the experiments reported in [BGP ${ }^{+}$09], however there it sufficed to stimulate just one $\mathrm{HC}$ neuron to suppress GDP generation. This might in part be explained by insufficient knowledge of 


\begin{tabular}{llr}
\hline Quantity & Description & Value \\
\hline$V_{\text {rest }}^{\mathrm{HC}}$ & resting potential of HC IN & $-66 \mathrm{mV}$ \\
$V_{\text {rest }}^{\mathrm{LC}}$ & resting potential of LC IN & $-64 \mathrm{mV}$ \\
$V_{\mathrm{th}}^{\mathrm{HC}}$ & threshold potential of HC IN & $-53 \mathrm{mV}$ \\
$V_{\mathrm{th}}^{\mathrm{LC}}$ & threshold potential of LC IN & $-39 \mathrm{mV}$ \\
$g_{\mathrm{l}}^{\mathrm{HC}}$ & leak conductance of HC IN & $2.64 \mathrm{nS}$ \\
$g_{1}^{\mathrm{LC}}$ & leak conductance of LC IN & $2.36 \mathrm{nS}$ \\
$C_{\mathrm{m}}^{\mathrm{HC}}$ & membrane capacitance of HC IN & $65 \mathrm{pF}$ \\
$C_{\mathrm{m}}^{\mathrm{LC}}$ & membrane capacitance of LC IN & $54 \mathrm{pF}$ \\
EPSP & EPSP of HC IN & $2.1 \mathrm{mV}$ \\
EPSP & EPSP of LC IN & $2.6 \mathrm{mV}$ \\
$I_{\text {stim }}$ & stimulation direct current & $40 \mathrm{pA}$ \\
& & \\
$E_{\mathrm{GABA}}$ & reversal potential of response to GABA & $-40 \mathrm{mV}$ \\
$E_{\text {exc }}$ & reversal potential of response to exc currents & $0 \mathrm{mV}$ \\
$\tau_{\text {syn,inh }}$ & synaptic time constant of interneurons & $0.5-0.7 \mathrm{~ms}$ \\
$\tau_{\text {syn,exc }}$ & synaptic time constant of pyramidal neurons & $0.4-0.7 \mathrm{~ms}$ \\
$g_{0}^{\mathrm{HC}}$ & conductance coefficient of HC IN & $22.7 \mathrm{nS}$ \\
$g_{0}^{\mathrm{LC}}$ & conductance coefficient of LC IN & $25.4 \mathrm{nS}$ \\
$\tau_{\text {ref }}$ & refractory time & $2 \mathrm{~ms}$ \\
\hline & &
\end{tabular}

Table 5.1: All parameters besides the last seven are directly taken from $\left[\mathrm{BGP}^{+} 09\right]$. $E_{\mathrm{GABA}}$ in INs in early development is reported to be between $-46 \mathrm{mV}$ and $-36 \mathrm{mV}$ in [BAGTK07].

\begin{tabular}{llr}
\hline Quantity & Description & Value \\
\hline$\tau_{\text {syn }}$ & time constant of PSC & $0.5-0.7 \mathrm{~ms}$ \\
$A$ & PSC amplitude & $10 \mathrm{pA}$ \\
$\tau_{\text {rec }}$ & time constant of recovery & $1000 \mathrm{~ms}$ \\
$\tau_{\text {fac }}$ & time constant of facilitation & $0 \mathrm{~ms}$ \\
$U$ & facilitation parameter & 0.5 \\
\hline
\end{tabular}

Table 5.2: Parameters for the short-term plasticity synapses. For a description of the model see [TPM98].

neuron parameters, the oversimplified neuron model itself, and also lack of knowledge about the actual underlying network structure. Only the functional network 
structure was estimated in the experiments by calcium imaging, which has limited resolution and might be biased towards strongly active neurons.

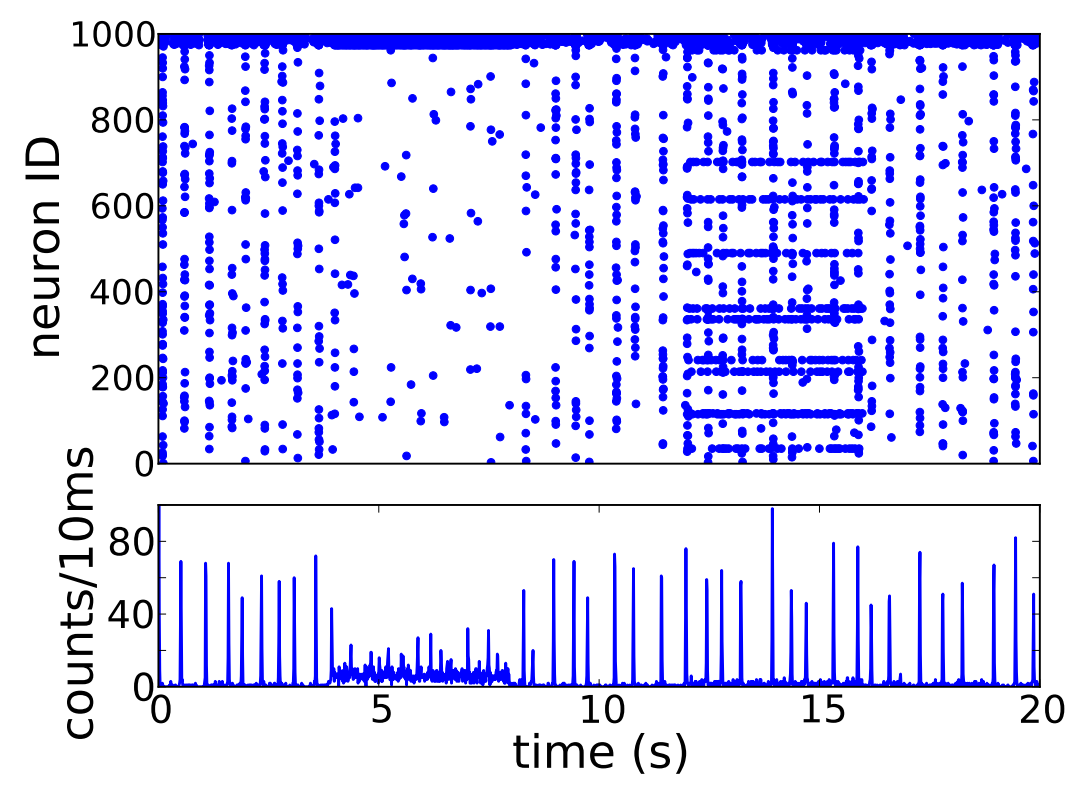

Figure 5.5: Depolarization of conductance-based leaky integrate-and-fire neurons. Spike activity (upper panel) and population activity (lower panel) of a network of size 1000. Neuron labeling is from low connectivity (LC) neurons (1) to high connectivity (HC) neurons (1000). Synchronous events are initiated in the HC part of the network and spread across the network. At $t=4 \mathrm{~s}$ to $t=8 \mathrm{~s}$ some of the $\mathrm{HC}$ neurons are depolarized by a current of $40 \mathrm{pA}$, synchronous events are suppressed, while a stimulation at $t=12 \mathrm{~s}$ to $t=16 \mathrm{~s}$ of some of the LC neurons slightly increases population rate but does not affect network synchrony. The rate of the Poisson input mimicking the pyramidal neuron input was chosen as $90 / \mathrm{s}$ to both LC and HC neurons weighted (the weight equals the area of the evoked PSP when stimulated by a unit $\delta$-pulse at resting potential) by $\operatorname{EPSP}^{\mathrm{LC}}$ and $\mathrm{EPSP}^{\mathrm{HC}}$, respectively.

The inter-GDP-interval distribution (see Fig. 5.6 (a)) was estimated from $500 \mathrm{~s}$ of ongoing (unperturbed) activity with parameters as in Fig. 5.5. Note, that the time scale is off: in the experiments the frequency of GDPs is about $0.01 / \mathrm{s}$, while in the simulation here it is approximately $2 / \mathrm{s}$.

For fixed network structure the GDP frequency is mainly determined by the parameters of the short-term depression of synapses. If e.g. the recovery time $\tau_{\text {rec }}$ is doubled, also the inter-GDP-interval distribution is shifted towards twice the mean inter-GDP-interval, cf. Fig. [5.6 (b).

GDPs are initialized in the HC subpopulation and spread to the LC population, see Fig. 5.7 (a). The same is observed in the experiments [BGP+09]. Moreover, 

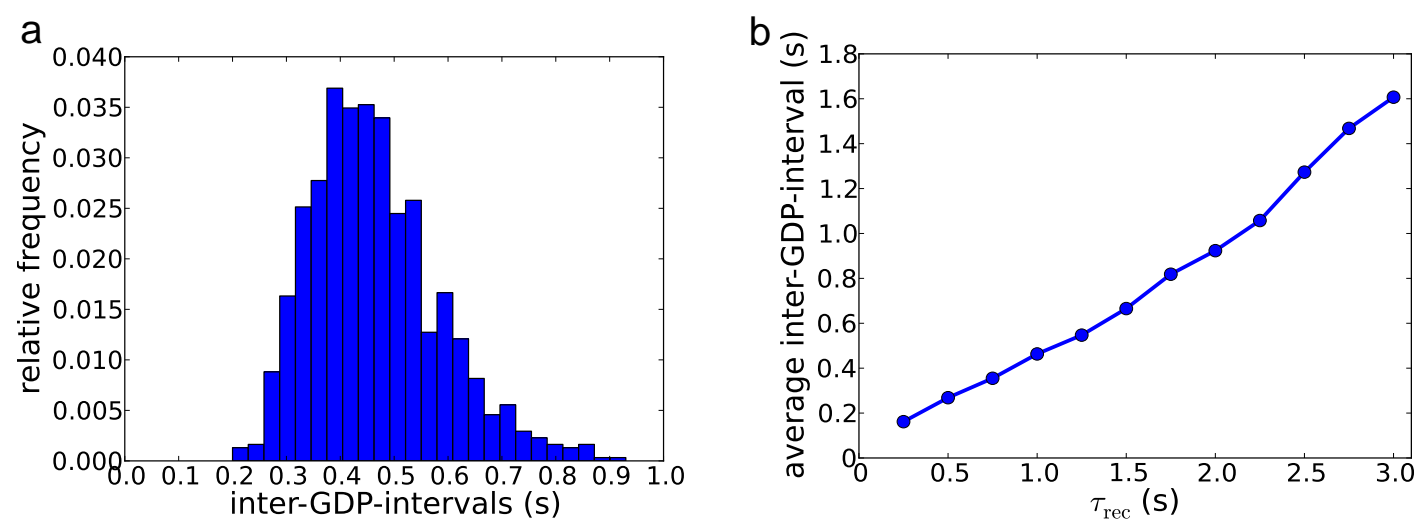

Figure 5.6: Inter-GDP-intervals. a: Distribution of inter-GDP-intervals estimated from $500 \mathrm{~s}$ of unperturbed activity of the network shown in Fig. 5.5. The mean value is at approximately half a second $(0.47 \mathrm{~s})$. b: If $\tau_{\text {rec }}$ is varied with all other parameters fixed we observe an almost linear relationship between the mean inter-GDP-interval and $\tau_{\text {rec }}$ with a slope $\sim 0.5$

spontaneous upward (depolarizing) fluctuations in the Poissonian pyramidal input can trigger GDPs, cf. Fig. 5.7 (b). Hence, intrinsically bursting pyramidal activity could be the actual pacemaker of GDP activity, facilitated by depolarizing INs (see Sip05).

Note, that the main difference between $\mathrm{HC}$ and $\mathrm{LC}$ neurons is the threshold value: that for $\mathrm{HC}$ neurons lies much closer to the resting potential of the cells and hence given the same effectively depolarizing input the $\mathrm{HC}$ cells are more likely to spike, i.e. more excitable than the LC cells. Indeed, in simulations we observe that though on average the membrane potential of the LC neurons is higher in absolute terms, the distance of this averages to the respective thresholds is larger in LC neurons than in HC neurons, see Fig. 5.8 (a).

\subsubsection{Mechanisms of GDP generation and suppression}

\section{GDP generation}

The underlying mechanism of GDP generation thus appears to be the following. Some HC neurons get activated by random fluctuations in the pyramidal input and send spikes to both other HC neurons as well as to the LC neurons. The other HC neurons respond first since they are closer to threshold. The massive $\mathrm{HC}$ activation then spreads to the LC neurons and causes some of them to spike. The synchronous event soon dies out due to synaptic depression and refractoriness. To underline the individual levels of processing in the neuron and its synapses Fig. 5.8 (b) shows the time course of the synaptic conductance $g_{\mathrm{inh}}(t)$, the membrane potential $V(t)$, the resulting current $I_{\text {syn,inh }}(t)$ and the depression of synaptic strength measured by $s_{\text {act }}(t)$ of an HC neuron during a GDP. 

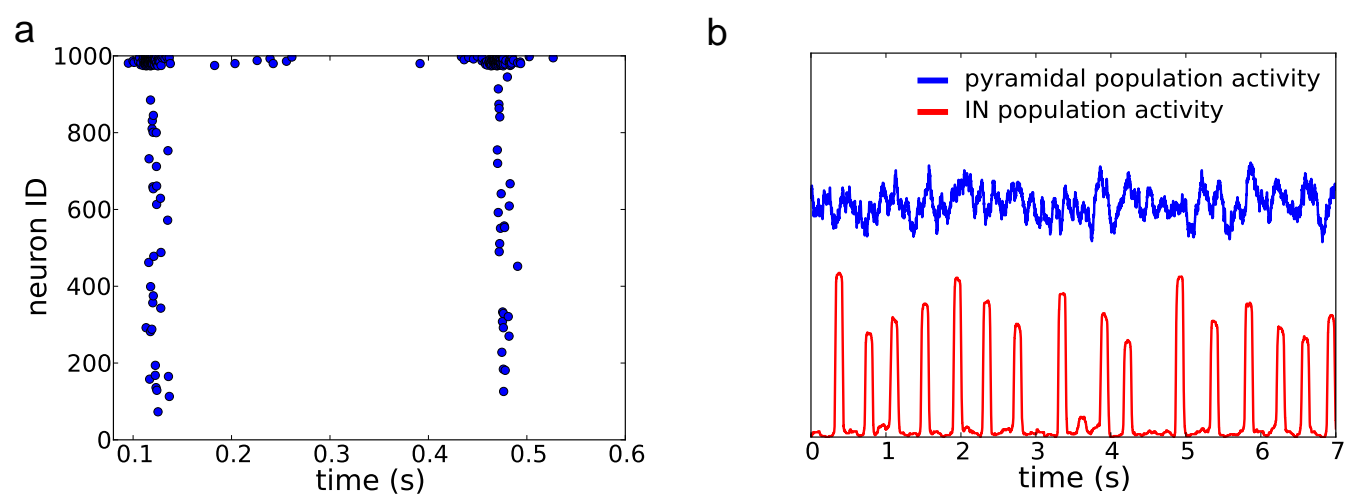

Figure 5.7: Initiation of GDPs. a: Raster plot of spike times. GDPs originate in the densely connected HC population (the uppermost 27 neurons) and spread to the rest of the network. This is also observed in the experiments by [BGP+09]. b: GDPs (red) mostly coincide with upwards fluctuations in the pyramidal population activity (blue). If these are exceptionally strong and close-by the inter-GDP-interval can be shorter than average, see e.g. at around $4 \mathrm{~s}$. The stronger synaptic depression leads to a longer next interval. Hence, it is to be expected that if the pyramidal activity was oscillating, that frequency could also act as a pacemaker for the GDP activity as suggested by [Sip05.

\section{GDP suppression}

When stimulated by a depolarizing direct current neurons fire at higher frequency. With each emitted spike, however, their synapses get more and more depressed (we excluded facilitation). In effect the stimulated neurons get decoupled from the rest of the network, and also those neurons that fire in response have in turn weaker impact on their post-synaptic target neurons the more they spike. This has a strong effect on GDP activity when HC neurons are stimulated, since they drive the network activity, while there is no noticeable effect when LC neurons are stimulated.

Though it is unclear if synaptic vesicle depletion is a determining factor for the inter-GDP-interval statistics, it is conceivable that other formally very similar recovery mechanisms with longer time constants play a role.

In the following we will now analyze the further role of network topology, neuron properties and synapse properties in the generation of GDPs.

\subsubsection{Random network topology}

Random networks (cf. also Section 4.5) are networks in which connections between any two different neurons are established in a Bernoulli-type fashion with a connection probability $P$, such that no neuron pair is considered more than once. The ensemble of network topologies that is generated in this way thus belongs to the class of fixed- $P$ Erdös-Rényi graphs. In the last section we saw that synchronous network 

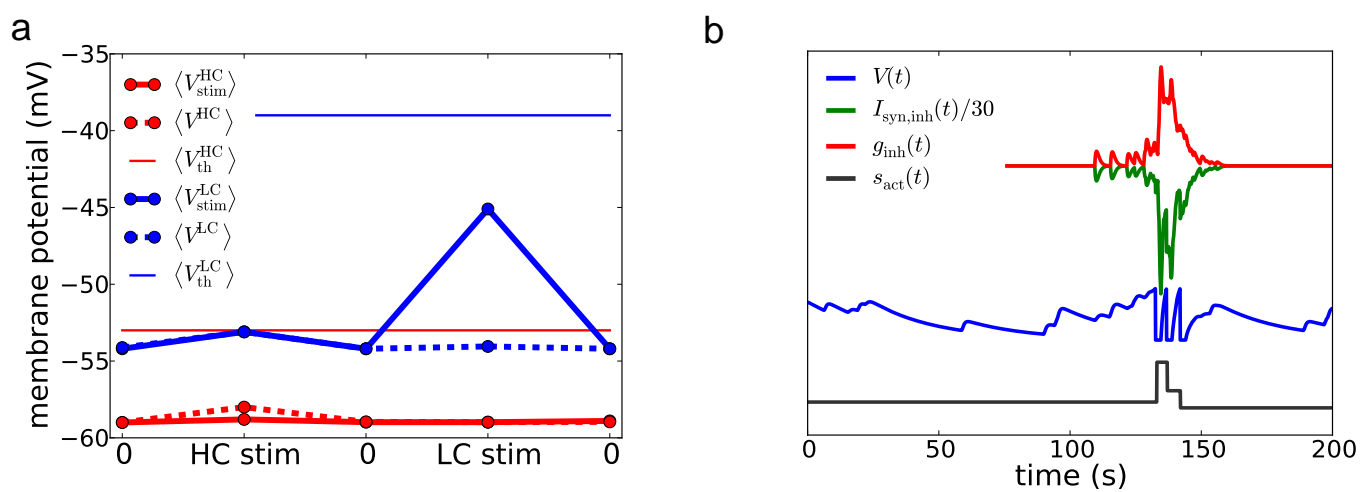

Figure 5.8: Temporal averages of membrane potentials. a: The mean membrane potential in all stimulation paradigms (' 0 ' stands for no stimulus applied, 'HC stim' and 'LC stim' for the case where some HC or LC neurons were depolarized, respectively) is much closer to threshold for HC neurons (red) than for LC neurons (blue). Moreover, the LC neurons are on average closer to the reversal potential $(-40 \mathrm{mV})$ than the $\mathrm{HC}$ neurons, hence $\left(V(t)-E_{\mathrm{GABA}}\right)$ is on average smaller for LC neurons. The closer $V(t)$ is to the reversal potential at the arrival time of a spike, the smaller $I_{\text {syn,inh }}$ that neuron receives. b: Individual example traces of the membrane potential (blue), conductance (red), resulting synaptic current (green) and synaptic activation variable (black) during a GDP. The more the neuron spikes, the more depressed its synaptic activation becomes in response.

events are generated in the dense part of the network, i.e. the $\mathrm{HC}$ subpopulation. Since the LC population is hence enslaved by the driving $\mathrm{HC}$ population and not actively taking part in GDP generation, we expect that random networks should show spontaneous synchronization as well, given they have a certain connection density $P$.

To analyze the impact of the slightly different neuron parameters in LC and HC neurons (cf. Table 5.4.2), we impose an additional constraint on the networks by fixing the in-degree of all neurons to the expectation value $k^{\text {in }}=P N$ exactly, similar to the condition already introduced in Section 5.3. Then the same fraction of neurons as before is randomly assigned to have HC-parameters, while the rest has LC-parameters.

Indeed, for a network of identical size $N=1000$ and number of HC-neurons as before, however random connectivity with probability $P=0.4$, stable network synchronization is observed (here $\tau_{\text {syn,inh }}=0.5 \mathrm{~ms}$ and $\tau_{\text {syn,exc }}=0.4 \mathrm{~ms}$ ). Moreover, the same depolarizing direct current stimulation as before can suppress GDPs, pointing towards the important role of the neuron parameter differences between $\mathrm{HC}$ and LC neurons. 


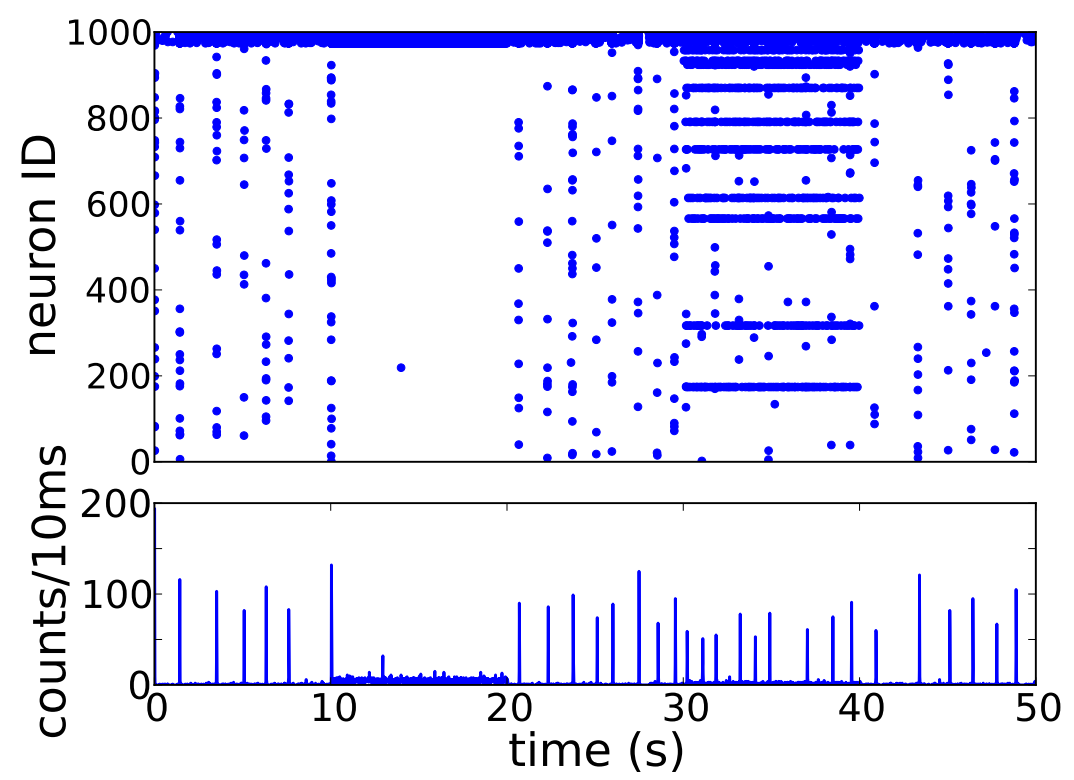

Figure 5.9: Depolarization of conductance-based leaky integrate-and-fire neurons in random networks. Spike activity (upper panel) and population activity (lower panel) of a random network of size 1000 with connection density $P=0.4$. Neuron labeling is from low-connectivity (LC) neurons (1) to high-connectivity (HC) neurons (1000). Synchronous events are initiated in the $\mathrm{HC}$ part of the network and spread across the network. At $t=10 \mathrm{~s}$ to $t=20 \mathrm{~s}$ some of the HC neurons are depolarized by a current of $40 \mathrm{pA}$, synchronous events are suppressed, while a stimulation at $t=30 \mathrm{~s}$ to $t=40 \mathrm{~s}$ of some of the LC neurons slightly increases population rate but does not affect network synchrony. The rate of the Poisson input mimicking the pyramidal neuron input was chosen as $110 / \mathrm{s}$ to both LC and HC neurons weighted by $\mathrm{EPSP}^{\mathrm{LC}}$ and $\mathrm{EPSP}^{\mathrm{HC}}$, respectively.

\subsubsection{Current-based neurons with $\delta$-shaped post-synaptic currents}

In current-based LIF neurons the impact of synaptic inputs on the membrane potential $V(t)$ does not depend on the value of $V(t)$ itself. Moreover, we assume postsynaptic currents in response to spike pulses to have no temporal extent, i.e. they are modeled as $\delta$-pulses. The subthreshold dynamics of the neuron is fully linear and governed by the differential equation

$$
\frac{d V(t)}{d t}=-\frac{V(t)}{\tau_{m}}+\frac{I_{\mathrm{syn}, \mathrm{exc}}+I_{\mathrm{syn}, \mathrm{inh}}}{C_{m}}
$$




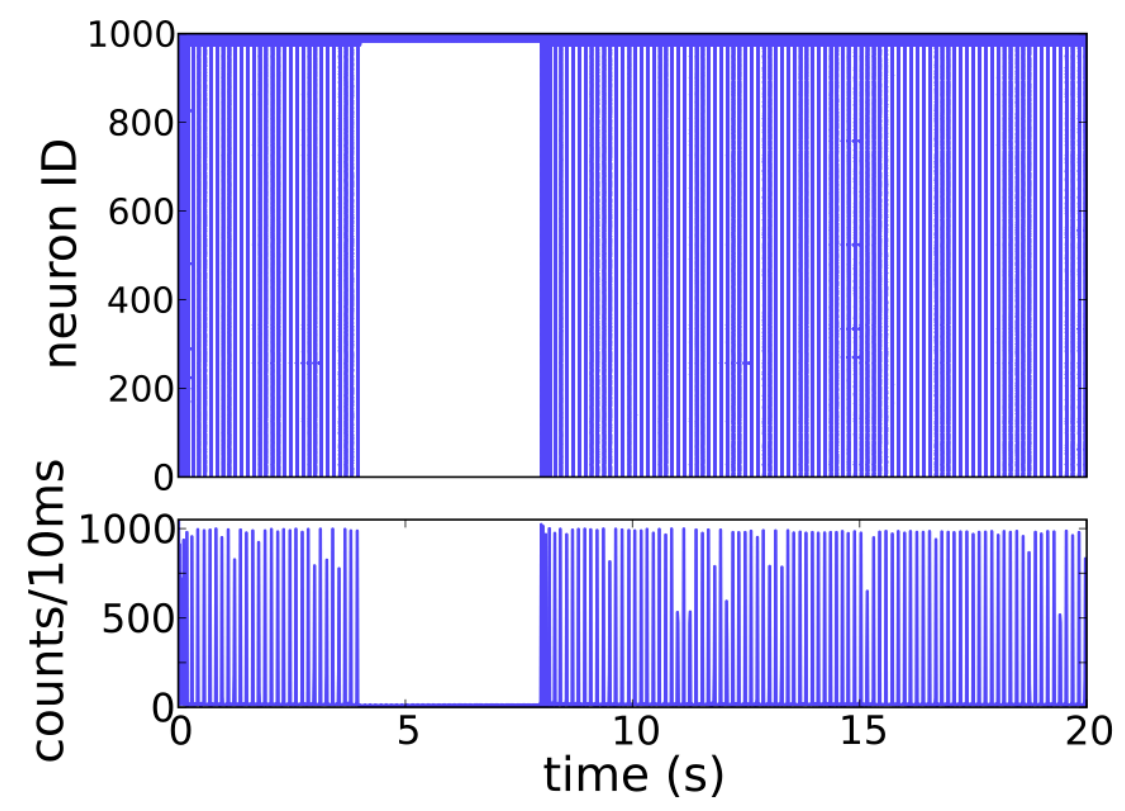

Figure 5.10: Hyperpolarization of current-based leaky integrate-and-fire neurons. Spike activity (upper panel) and population activity (lower panel) of the same network as in Fig.5.5. At $t=4 \mathrm{~s}$ to $t=8 \mathrm{~s}$ some of the $\mathrm{HC}$ neurons are hyperpolarized by a current of $-40 \mathrm{pA}$, synchronous events are suppressed, while a stimulation at $t=12 \mathrm{~s}$ to $t=16 \mathrm{~s}$ of some of the LC neurons does not affect network synchrony. The excitatory input current was a direct current.

with

$$
\begin{aligned}
& I_{\mathrm{syn}, \mathrm{exc}}=C_{m} J_{\mathrm{exc}} \sum_{j \in \mathrm{exc}} \delta\left(t-t_{j, k}-d\right) \\
& I_{\mathrm{syn}, \text { inh }}=C_{m} J_{\mathrm{inh}} \sum_{j \in \mathrm{inh}} \delta\left(t-t_{j, k}-d\right)
\end{aligned}
$$

with post-synaptic potential amplitudes $J_{\text {inh/exc }}$ in $\mathrm{mV}$, the delay $d$, and the membrane time constant $\tau_{m}=C_{m} / g_{\text {leak }} . t_{j, k}$ denotes the $k$-th spike time of input neuron $j$. To keep activity levels similar and mimic the effectively excitatory impact of the GABA-ergic neurons in early development, we choose the coupling strengths $J_{\text {inh }}^{\mathrm{HC}}=2.27$ and $J_{\text {inh }}^{\mathrm{LC}}=2.54$. All other parameters were the same as given in Table (5.4.2). The pyramidal input is modeled as a depolarizing direct current as well, such that in effect the neurons are LIF oscillators, coupled however by synapses with short-term plasticity.

Simulations show that a fast synchronous mode arises that is only kept from exploding by synaptic depression, see Fig. 5.10, Stimulation with a depolarizing direct current has no suppressing effect on this mode, however a hyperpolarizing current applied to HC neurons can in fact suppress synchronous activity, while the same stimulus to the LC neurons does not (Fig (5.10), given the pyramidal 
depolarizing input current is chosen adequately.

The mechanism behind this is similar to the one discussed in Section 5.4.1: the $\mathrm{HC}$ neurons are driver neurons due to their higher rate in response to the same excitatory input, while the LC neurons are more hyperpolarized and - if adjusted like this - need the $\mathrm{HC}$ input spikes to cross threshold at all. If a depolarizing current shuts off part of this drive, the LC neurons stay below threshold and GDP events do not occur.

\subsection{Summary and discussion}

In this Chapter, we first investigated single-variable pulse-coupled oscillators where the question about possible mechanisms that remove oscillations in the neurobiological system translates into the question about possible mechanisms destroying a close-to-synchronous periodic orbit [DTD $\left.{ }^{+} 04\right]$. We numerically made first observations that driving hub neurons do indeed have more impact on the phase spread than driving a non-hub neuron. The maximal induced phase spread, however, is about equal in both cases. Thus, the theoretical framework based on previous works DTD $^{+}$04, Tim07] does not suffice to explain the experimentally observed phenomena. Nevertheless, our simulations support the hypothesis that driving single neurons in a pulse-coupled system may induce effects of local heterogeneity similar to those obtained for continuous time phase-coupled oscillators in [Tim07]. In order to be able to obtain analytical results, we will have to slightly modify the proposed framework. We may exclude the phase of the driven neuron from the phases considered for the determination of the maximal phase differences (see Fig. 5.4, b). Alternatively, we have to slightly modify the model, e.g. by incorporating a refractory period as in [RL11].

To better understand the effects observed by $\left[\mathrm{BGP}^{+} 09\right]$ we then investigated a slightly more realistic modeling approach that takes into account temporally extended synaptic inputs modeled as conductances. In this way we could include the basic observation from early developing hippocampal networks that GABA a neurotransmitter that in mature networks mediates inhibition - can depolarize immature cells. This is due to the fact that at very early stages of neuron development the corresponding channel has a much higher reversal potential than at later stages. We moreover assumed synapses with short-term plasticity, such that whenever a spike is transmitted recovery to full transmission capability takes a finite time. This mimics the depletion of synaptic vesicles which contain neurotransmitter and need to be recreated after each activation of the synapse.

Including the various neuron parameters that Bonifazi et al. $\left[\mathrm{BGP}^{+} 09\right]$ estimated from their experiments into our simulation leads to very similar GDP activity as observed in the experiments, and most of all the same stimulus that was used to suppress GDPs there accomplished suppression in the simulations as well. However, the inter-GDP-interval turned out to be much shorter than observed in experiments. This point can however be resolved by increasing $\tau_{\text {rec }}$ in the synaptic dynamics.

Judging the validity of our results is not straight-forward since a lot of further assumptions, besides synaptic depression, went into the set-up of the simulation: 
e.g. the size of conductance amplitudes $g_{0}$ (cf. Eq.5.4.3), the total input from the pyramidal population encoded by its rate, or the synaptic time constants. The latter turn out to be quite small $\sim 0.5 \mathrm{~ms}$. If they are larger, GDP generation breaks down and activity becomes irregular. Increasing the synaptic time constant also likely increases the net current that is transmitted. A rescaling of the evoked postsynaptic potential by rescaling $g_{0}$ (cf. Eq. 5.4.3) could resolve part of this problem, however in the highly nonlinear conductance-based neuron this is not a straightforward task. Spiking activity also becomes irregular when the rate of the pyramidal population becomes too high. Further investigation is necessary to understand how these findings relate to biology, however, mature neuronal networks typically have asynchronous irregular spiking activity, so it is interesting to speculate that these observations relate to the changes in synaptic gain and pyramidal input during cell maturation and network development [BAGTK07].

One further shortcoming of our modeling results so far is the fact that in experiments it suffices to stimulate a single HC neuron to suppress GDPs. In our simulation runs we needed to stimulate at least 5 neurons to obtain the same effect. We used the average neuron parameter values reported in [BGP$\left.{ }^{+} 09\right]$. Introducing more variation in these parameters might partially resolve this issue.

The main finding of this Chapter is that it is indeed rather the differences in neuron parameters, most of all in the effective distance to firing threshold, that make HC neurons so important for network dynamics. Topology plays a minor role, since simulations of random networks lead to similar results as those of scale-free networks. However, the random networks needed to be quite dense in comparison to scale-free networks and even in comparison to fully mature networks. Especially, in early network development connectivity is still sparse, so assuming that GDPs play a pivotal role in this phase, sparse scale-free structures are a low-wiring cost solution.

We further mapped the main GDP generation mechanism - HC neurons drive the otherwise mostly silent LC neurons to fire whenever there is a spontaneous activation in the densely connected $\mathrm{HC}$ subpopulation - back to oscillator neurons that are connected by synapses with activity dependent short-term depression. There, a hyperpolarizing current is able to shut down the $\mathrm{HC}$ drive and suppress global network synchronization. Preliminary work suggests that one can map the mechanism to an essentially two-dimensional oscillator problem. To further formalize and mathematically analyze that reduced modeling approach is work in progress.

Though such a more general model might have interesting applications in many real-world phenomena, a biologically motivated model such as the network of conductance-based neurons is of much help in actually interpreting and understanding the specific neuroscientific system at hand, and continuation of the analysis of this more complex model is in progress as well. 


\section{Conclusions}

In this thesis, we studied the impact of complex network topologies on the synchronization dynamics of coupled oscillators. We investigated different oscillator types, intrinsic dynamics and coupling schemes: phase oscillators coupled via phase differences, higher-dimensional periodic systems coupled diffusively as well as neural circuits with inhibitory delayed pulse-coupling. All these dynamical systems are idealizations of systems occurring in nature.

To provide an immediate application of the work in this thesis we investigated more realistic neuron models, in particular current-based and conductance-based leaky integrate-and-fire point oscillators in order to explain recent experimental findings.

The work presented in the thesis hinges on three main questions:

1. How does the speed of synchronization depend on network topology? On the spectrum from completely ordered grid-like networks, intermediately ordered networks in the small world regime to completely unordered random networks, which class of topologies is most conducive to synchronization?

2. Can the topological features of small-world networks be predicted analytically, thereby bypassing the enormous computational effort required in constructing these networks?

3. What role do hub neurons in a network with scale-free topology play in the emergence and destruction of globally synchronous oscillations?

The answers to these questions are supplemented by theoretical work to derive a mean field approximation for calculating the spectra of networks in the small-world regime. This work has wide ranging applicability outside the context of this thesis given enormous importance of small-world networks in biology, physics, engineering and the social sciences which in the past had to rely on numerical efforts alone.

In the first part (Chapter 3), we investigated the impact of topology on the speed of synchronization of various oscillator types, intrinsic dynamics and coupling schemes: phase oscillators coupled via phase differences, higher-dimensional periodic systems coupled diffusively as well as neural circuits with inhibitory delayed pulse-coupling and consistently found qualitatively the same results. We derived analytical predictions for the asymptotic synchronization times, including an extension of the master stability function to determine the synchronization speed of the system.

We found that small-world networks never synchronize fastest. This result is surprising as previous workcite [BP02, WS98] suggested that small-world networks might be particularly supportive to synchronization. Our results however show that regarding speed of synchronization they are not special at all.

In particular, in network ensembles with fixed average path length, synchrony is consistently fast for regular rings, fastest for completely random networks, and 
slowest in the intermediate small-world regime. It is an astonishing result that holds across various oscillator types, intrinsic dynamics and coupling schemes: phase oscillators coupled via phase differences, higher-dimensional periodic systems coupled diffusively as well as neural circuits with inhibitory delayed pulse-coupling. Thus, our results indicate that this is a universal phenomenon. Our investigations of realworld networks support this view. Although the considered networks may be in the small-world regime already, rewiring still strongly increases the synchronization speed, even for ensembles with fixed average path length. It remains an open question why rewiring typically implies faster synchronization. In addition, we could extend our analysis of the asymptotic synchronization time to additional other time scales relevant for the system's function or dysfunction, respectively.

In Section 3.5.5 we found a surprising similarity between Kuramoto and pulsecoupled oscillators. An interesting question poses itself under which conditions the synchronization times of the former can approximated by or even be mapped onto those of the latter.

In the second part (Chapter 4), we introduced a simple two-stage mean field rewiring to analytically derive predictions for the spectra of graph Laplacians. Systematic numerical checks confirm that our prediction is accurate for the second largest eigenvalue and even for the smallest eigenvalue for all parameter values except for small degrees or very high topological randomness of the order of unity for the second largest eigenvalue and up to $q$-values corresponding to the small-world regime for the smallest eigenvalue, respectively. For smaller $k$, our analytic prediction still serves as a valuable guide for the overall dependence on all topological parameters. For $q$ close to unity, our mean field predictions are well complemented by standard random matrix theory. Besides the second largest and smallest eigenvalues that give valuable information about initial and asymptotic relaxation dynamics, the bulk spectrum and the fine structure of the spectrum are also well approximated by our analytical prediction.

In particular, the spectral prediction includes regular rings, small worlds, and substantially more randomly rewired networks and undirected as well as directed ones. Thus, our theoretical predictions agree well with the eigenvalues obtained numerically over almost the entire range of topological randomness $q$, thereby improving upon previous attempts based on perturbation theory for $q \ll 1$ [Mon99, BP02].

Interestingly, eigenvalues for directed networks are approximated more accurately and in a wider range of $q$-values, in spite of the mean field rewiring being undirected. This is in particular related to the fact that the predictions for the undirected second-largest eigenvalues at $q=1$ are larger in real part than the directed ones, while all the mean field eigenvalues converge to the eigenvalue -1 at $q=1$.

The simple mean field approach presented here definitely leads to an essential reduction of computational efforts when studying randomized (regular or smallworld) network models. As a future task it may be extended to rewiring approaches starting from other than ring-like structures, e.g. to two or three dimensions, as for instance, relevant for neural network modeling [SB09]. Checking with appropriate models, it may thus serve as a powerful tool to predict the relations between structural and dynamical properties of randomized networks.

The observed decreasing accuracy of the mean field approximation for 'small' $k$ - 
values leads to an interesting task for further work. We can apply our rewiring on average to the slightly modified Watts-Strogatz small-world model [NW99]. Here, instead of rewiring existing links, extra links, often called shortcuts are added between pairs of nodes chosen at random, but no links are removed from the initial ring structure. This may lead to a better approximation for low connectivity since it is not possible to destroy the initial ring structure.

In the third part (Chapter 5), we first reviewed the experimental findings with the emphasis on the reported phenomenon that stimulating a single hub neuron may completely remove global collective oscillations, while driving a non-hub neuron the same way does not significantly affect the oscillations. Furthermore, we suggested an approach to implement the discovered scale-free topology in our modeling.

Firstly, using the abstract single-variable pulse-coupled oscillators the question about possible mechanisms that remove oscillations in the neurobiological system translates into the question: What are possible mechanisms for destroying a closeto-synchronous periodic orbit $\left[\mathrm{DTD}^{+} 04\right]$ ? Numerically, we confirmed that driving a hub neuron does indeed have more impact on the phase spread than driving a nonhub neuron. The maximal induced phase spread, however, is about equal in both cases. Thus, the theoretical framework based on previous works [DTD ${ }^{+}$04, Tim07] does not suffice to explain the experimentally observed phenomena. Nevertheless, our simulations support the hypothesis that driving single neurons in a pulse-coupled system may induce effects of local heterogeneity similar to those obtained for continuous time phase-coupled oscillators in [Tim07].

Secondly, we investigated biophysically more realistic models to uncover possible mechanisms. Indeed, we could switch off global oscillations by targeted stimulation of hub neurons - which does not work for non-hub neurons - and thus reproduced parts of the experimental phenomena. In this context, the main finding is that the variety of neuron parameters, in particular the effective distance to firing threshold, that make HC neurons so important for network dynamics. Furthermore, our results suggest that the topology plays a minor role. We mapped the main GDP generation mechanism - HC neurons drive the otherwise mostly silent LC neurons to fire whenever there is a spontaneous activation in the densely connected $\mathrm{HC}$ subpopulation - back to oscillator neurons that are connected by synapses with activity dependent short-term depression. There, a hyperpolarizing current is able to shut down the HC drive and suppress global network synchronization. Preliminary work suggests that one can map this mechanism to an two-dimensional oscillator problem.

In this thesis we were only able to present a snapshot of the work on the role of hub neurons. Ongoing work is progressing along two axes; firstly, a reduced model is analyzed mathematically which could have interesting applications outside of the neuroscientific context. At the same time the more realistic model of conductancebased neurons is studied in order to provide insight into the experimentally observed phenomena of globally synchronous oscillations. There remain many problems to be solved, but a promising basis for future studies in this direction has been established. 
If the brain were simple enough for us to understand it, we would be too simple to understand it. 


\section{Acknowledgements}

First of all, I would like to thank my thesis supervisor Marc Timme for giving me the chance of pursuing my $\mathrm{PhD}$ degree in his group at the Max-Planck-Institute for Dynamics and Self-Organization. He has always been motivating and encouraging in our discussions.

Special thanks are due to Stefan Grosskinsky with whom I had a great scientific collaboration during my whole PhD time and to Birgit Kriener for the collaboration at the end which will hopefully be continued. In this context, also special thanks to Alessandro Torcini who initated the 'hub project' together with Marc and to Tsampikos Kottos and Zin Lin for many discussions.

Particularly, I also want to thank Markus Diesmann who made it possible that I experienced an unforgettable time at the Computational Neurophysics Research Unit at RIKEN Brain Science Institute, Wako City, Japan and all the people I got to know in Japan, especially Larissa Albantakis, Danielle Corbett, Michael Denker, Tomoe Furuya, Sonja Grün, Adrian Ponce, Reiko Kiyotaki, Susanne Kunkel, Abigail Morrison and all the others I have unintentionally forgotten here. I would also like to thank the members of my thesis committee Prof. Dr. Stephan Herminghaus and Prof. Dr. Annette Zippelius, who agreed to be the second reviewer of this thesis.

I furthermore acknowledge numerous helpful discussions - both scientific and private - with my colleagues from the institute, namely Ghazaleh Afshar, Demian Battaglia, Yorck-Fabian Beensen, Oliver Bendix, Dima Bibichkov, Christian Bick, Armin Biess, Kai Bröking, Nikolai Chapochnikov, Ahmed El Hady, Stephan Eule, Katja Fiedler, Frederik Fix, Harold Gutch, David Hofmann, Sven Jahnke, Wolfgang Keil, Hinrich Kielblock, Christoph Kirst, Jakob Metzger, Jan Nagler, Martin Rohden, Fabio Schittler Neves, Andreas Sorge, Olav Stetter, Mitja Tsigankov, Frank Van Bussel, Heike Vester, Gunter Weber and all the others I have unintentionally forgotten here.

And special thanks goes to the crew of the networks dynamics group and the institute as a whole, who always care for a friendly atmosphere and a great support in everyday life - in particular, to Theo Geisel for creating a very special environment at our institute.

I would also like to thank my special friends through university Michael Köhn, Christian Reisswig, Svenja Riekeberg and Torsten Weber. In particular, Michael Köhn is close by my side now from school on: Thanks a lot for everything we have experienced together yet (to be continued...).

Thanks a lot to Freya Brauckmann and Eric Stellamanns with whom I could talk about all the stuff not related to science and for all the nice time together in Göttingen.

On the personal side, I am much obliged to my parents and sisters, who supported me during my whole life, as well as to Katharina for her love, understanding and support. 



\section{Curriculum Vitae}

\begin{tabular}{|c|c|}
\hline $\begin{array}{r}\text { Name: } \\
\text { Citizenship: } \\
\text { Date of Birth: } \\
\text { Marital Status: }\end{array}$ & $\begin{array}{l}\text { Carsten Grabow } \\
\text { German } \\
1980 / 06 / 17 \\
\text { not married }\end{array}$ \\
\hline Academic Degrees: & $\begin{array}{l}\text { Diploma in Physics, } 2007, \\
\text { overall grade: excellent, } \\
\text { Institute for Theoretical Physics, } \\
\text { Leibniz University Hannover }\end{array}$ \\
\hline Current Position: & $\begin{array}{l}\text { PhD Student, } \\
\text { Network Dynamics Group, } \\
\text { Max Planck Institute for Dynamics } \\
\text { and Self-Organization, Göttingen. } \\
\text { Supervisor: Prof. Dr. M. Timme }\end{array}$ \\
\hline \multirow[t]{4}{*}{ Publications: } & $\begin{array}{l}\text { Carsten Grabow, Stefan Grosskinsky and Marc Timme } \\
\text { Small-world spectra in mean field theory } \\
\text { [arXiv:1112.1728]. }\end{array}$ \\
\hline & $\begin{array}{l}\text { Carsten Grabow, Stefan Grosskinsky and Marc Timme } \\
\text { * Speed of complex network synchronization } \\
\text { Eur. Phys. J. B } 84(2011)(4) \\
613-626 \text { [arXiv:1106.4337]. }\end{array}$ \\
\hline & $\begin{array}{l}\text { Carsten Grabow, Steven Hill, Stefan Grosskinsky and Marc Timme } \\
\text { Do small worlds synchronize fastest? } \\
\text { Europhys. Lett. } 90(2010)(4) \\
48.002 \text { [arXiv:1005.3757]. }\end{array}$ \\
\hline & $\begin{array}{l}\text { Michael Flohr, Carsten Grabow and Michael Koehn } \\
\text { Fermionic expressions for the characters } \\
\text { of } c_{p, 1} \text { logarithmic conformal field theories } \\
\text { Nuclear Physics B } 768(2007) \\
263-276 \text { [arXiv:0611.241] }\end{array}$ \\
\hline \multicolumn{2}{|c|}{$\begin{array}{l}\text { *see: The European Physical Journal Highlight Paper of July } 2011 \text { press coverage: } \\
\text { USA: Cell }(\text { DNA), Springer Select, PhysOrg, ScienceNewsLine (Technology); UK: ScienceDaily, } \\
\text { New Zealand: InterestingTech; India: Phodphad; Nepal: Science News; } \\
\text { Internet Services: Twitter.com; Facebook.com, KurzweilAI; AlphaGalileo.org; Fuse.TV; } \\
\text { TechScienceNews.com; plus news reports in about } 60 \text { other blog websites. }\end{array}$} \\
\hline
\end{tabular}


Professional Education:

Apr. 2008

Graduate Student,

Max Planck Institute for Dynamics

and Self-Organization, Göttingen

and Georg August University Göttingen.

Supervisor: Prof. Dr. M. Timme.

Oct. 2001 - Mar. 2007 Studies of Physics,

Leibniz University Hannover.

Diploma thesis supervisor: PD Dr. M. Flohr.

Thesis title: Fermionic Sum Representations of Characters

in Logarithmic Conformal Field Theory.

Fellowships:

2009 Japan Society for the Promotion of Science Stipend

RIKEN Brain Science Institute, Wako-shi, Tokyo, Japan.

School Education:

1993 - 2000 High School,

Herzog-Ernst-Gymnasium Uelzen.

1987 - 1993 Elementary School and Orientation Stage,

Hermann-Löns-Schule Uelzen.

Community Service:

$2000-2001$ St. Marien Church, Uelzen. 


\section{List of Figures}

2.1 Strongly connected components . . . . . . . . . . . . . . . . . . . . . . 17

2.2 Clustering in undirected networks. . . . . . . . . . . . . . . . . 18

2.3 Clustering in directed networks . . . . . . . . . . . . . . . . . . . . 19

2.4 Rewiring directed networks . . . . . . . . . . . . . . . . . 21

2.5 Small worlds . . . . . . . . . . . . . . . . . . . . 21

3.1 Time scales of svnchronization of oscillator networks. . . . . . . . . 28

3.2 Quantifving the small-world regime. . . . . . . . . . . . . . . . 30

3.3 Master stability functions for the Rössler oscillators. . . . . . . . . . . 33

3.4 The eigenvalue distribution of the graph Laplacian. . . . . . . . . . 34

3.5 Comparing specific network ensembles. . . . . . . . . . . . . . . . 40

3.6 Monotonicitv: network ensembles with fixed in-degree. . . . . . . . 41

3.7 Non-monotonicity: network ensembles with fixed average path length. 43

3.8 Chaotic Rössler oscillators with fixed average path length. . . . . . 44

3.9 No crossing of the small-world regime for fixed betweenness centrality. 45

3.10 Universal nonlinear dependence. . . . . . . . . . . . . . . . . 47

3.11 Structures of the two relevant matrices $G$ and $\Lambda$. . . . . . . . . 48

3.12 Randomizing real-world networks. . . . . . . . . . . . . . . . . 49

3.13 Synchronization speed of real-world networks. . . . . . . . . . . 50

4.1 Rewiring 'on average' . . . . . . . . . . . . . . . . . 54

4.2 The banded structure of the mean field graph Laplacian. . . . . . . 57

4.3 Interpolating the eigenvalues . . . . . . . . . . . . . . . . . . 59

$4.4 \tilde{\lambda}_{2}^{\mathrm{mf}}\left(=\tilde{\lambda}_{\Delta}^{\mathrm{mf}}\right)$ alwavs constitutes the second largest eigenvalue. . . . . 60

4.5 Important points of the function $f(x) \ldots \ldots$. . . . . . . . . . 61

4.6 Second largest eigenvalues from regular to random networks. . . . . 63

4.7 Fixed network size . . . . . . . . . . . . . . . . . 64

4.8 Dependence on edge density and network size. . . . . . . . . . . . 65

4.9 Smallest eigenvalues from regular to random networks. . . . . . . . 66

4.10 Second largest eigenvalues for random networks. . . . . . . . . . . . 70

4.11 Analytics predicts structure of entire spectrum. . . . . . . . . . . . 72

5.1 Scale-free topology in the developing hippocampus. . . . . . . . . 77

5.2 Stimulation of HC but not LC neurons affects network dynamics. . $\quad 78$

5.3 Induced phase spreads. . . . . . . . . . . . . . . . . . . . . . . 80

5.4 Induced maximal phase differences. . . . . . . . . . . . . . . . . . . . . . . 81

5.5 Depolarization of cLIF neurons. . . . . . . . . . . . . . . . . 85

5.6 Inter-GDP-intervals $\ldots \ldots \ldots$. . . . . . . . . . . 86

5.7 Initiation of GDPs. $\ldots \ldots \ldots$. . . . . . . . . . . . 87 
5.8 Temporal averages of membrane potentials. . . . . . . . . . . . . 88

5.9 Depolarization of cLIF neurons in random networks. . . . . . . . . . 89

5.10 Hyperpolarization of current-based LIF neurons. . . . . . . . . . . . 90 


\section{Bibliography}

[AB00] R. AlBerT and A. L. BARABÁSI Topology of evolving networks: local events and universality Phys. Rev. Lett. 85 (2000)(24) 5234-5237.

[AB02] R. Albert and A. Barabasi Statistical mechanics of complex networks Rev. Mod. Phys. 74 (2002) 47-97.

$\left[\mathrm{ABV}^{+} 05\right] \quad$ J. Acebron, L. Bonilla, C. Vicente, F. Ritort and R. SPIGLER The Kuramoto model: A simple paradigm for synchronization phenomena Rev. Mod. Phys. 77 (2005)(1) 137-185.

[AC05] R. Agaev and P. Chebotarev On the spectra of nonsymmetric Laplacian matrices Linear Algebra Appl. 399 (2005) 157-168.

[ADGPV06] A. Arenas, A. Díaz-Guilera and C. J. Pérez-Vicente Synchronization Reveals Topological Scales in Complex Networks Phys. Rev. Lett. 96 (2006)(11) 114.102.

[ADK $\left.{ }^{+} 08\right]$ A. Arenas, A. Diazguilera, J. Kurths, Y. Moreno and C. ZHOU Synchronization in complex networks Phys. Rep. 469 (2008)(3) 93-153.

[ASBS00] L. Amaral, A. Scala, M. Barthelemy and H. Stanley Classes of small-world networks Proc. Natl. Acad. Sci. U.S.A. 97 (2000)(21) 11.149-11.152.

[ASW $\left.{ }^{+} 06\right]$ S. Achard, R. Salvador, B. Whitcher, J. Suckling and E. Bullmore A resilient, low-frequency, small-world human brain functional network with highly connected association cortical hubs J. Neurosci. 26 (2006)(1) 63.

[AY92] T. B. Achacoso and W. Yamamoto $A Y$ 's Neuroanatomy of $C$. Elegans for Computation (CRC Press, Boca Raton, FL, 1992).

[BAGTK07] Y. Ben-Ari, J.-L. Gaiarsa, R. Tyzio and R. Khazipov GABA: A pioneer transmitter that excites immature neurons and generates primitive oscillations Physiological Reviews 87 (2007)(4) $1215-1284$.

[Bal01] P. BALL The self-made tapestry: pattern formation in nature (Oxford University Press, USA, 2001). 
[BCDlR10] M. Buchanan, G. Caldarelli and P. De Los Rios Networks in Cell Biology (Cambridge University Press, 2010).

$\left[\mathrm{BGP}^{+}\right.$09] P. Bonifazi, M. Goldin, M. A. Picardo, I. Jorquera,

A. Cattani, G. Bianconi, A. Represa, Y. Ben-Ari and R. Cossart GABAergic Hub Neurons Orchestrate Synchrony in Developing Hippocampal Networks Science 326 (2009)(5958) 1419-1424.

[BH99] N. BRUneL and V. HAKIM Fast global oscillations in networks of integrate-and-fire neurons with low firing rates Neural Computation 11 (1999)(7) 1621-1671.

[BH09] A. Brouwer and W. HaEmers Spectra of graphs (Springer, 2009).

[BHG06] D. Brockmann, L. Hufnagel and T. Geisel The scaling laws of human travel Nature 439 (2006)(7075) 462-465.

[BJ08] A. BANERJEE and J. JOST On the spectrum of the normalized graph Laplacian Linear Algebra Appl. 428 (2008)(11-12) 3015-3022.

[BJL01] E. Ben-JACOB and H. Levine The artistry of nature Nature 409 (2001)(6823) 985-986.

[BKmr00] A. Broder, R. Kumar, F. Maghoul and P. Raghavan Graph structure in the web Comput. Netw. 32 (2000)(309-320).

[BL07] T. BIYIKOGLU and J. LEYDOLD Laplacian eigenvectors of graphs volume 1915 (Springer LNM, 2007).

[Boc08] S. BocCALETTI The synchronized dynamics of complex systems (Elsevier Science, 2008).

[Bol98] B. Bollobás Modern Graph Theory (Springer, New York, 1998).

[Bol01] B. Bollobás Random Graphs (Cambridge University Press, 2001).

[BP97] R. BADII and A. Politi Complexity: Hierarchical Structures and Scaling in Physics (Cambridge University Press, 1997).

[BP02] M. BARAhona and L. M. PeCORA Synchronization in Small-World Systems Phys. Rev. Lett. 89 (2002)(5) 054.101.

[BR88] A. BRAY and G. RodGERS Diffusion in a sparsely connected space: A model for glassy relaxation Phys. Rev. B 38 (1988)(16) 11.461.

[Bre10] M. BREDE Optimal synchronization on strongly connected directed networks Eur. Phys. J. B 74 (2010)(2) 217-225.

[Buz06] G. Buzsaki Rhythms of the Brain (Oxford University Press, 2006). 
[BW00] A. BARRAT and M. WeIGT On the properties of small-world network models Eur. Phys. J. B 13 (2000) 547-560.

[Cal07] G. CALDARELli Scale-free networks (Oxford University Press, USA, 2007).

[CDS80] M. Cvetcović, M. Doob and H. Sachs Spectra of Graphs Theory and Application (Academic Press, 1980).

[CHQ $\left.{ }^{+} 08\right]$ L. Chen, H. B. Huang, G. X. Qi, P. Luo, C. Qiu, X. D. ZhaO and Y. WANG Searching good indicators for predicting the synchronizability of heterogeneous networks and beyond Europhys. Lett. 84 (2008)(5) 50.003.

[Chu05] F. Chung Laplacians and the Cheeger inequality for directed graphs Annals of combinatorics 9 (2005)(1) 1-19.

[CP04] R. CROSS and A. PARKER The hidden power of social networks understanding how work really gets done in organizations (Harvard Business School Press, 2004).

[CPSV07] V. Colizza, R. Pastor-Satorras and A. Vespignani Reaction-diffusion processes and metapopulation models in heterogeneous networks Nature Physics 3 (2007)(4) 276-282.

$\left[\mathrm{CQH}^{+} 10\right]$ L. Chen, C. Qiu, H. B. Huang, G. X. QI and H. J. Wang Facilitated synchronization of complex networks through a discontinuous coupling strategy The European Physical Journal B 76 (2010)(4) 625-635.

[DA01] P. DAYAN and L. F. ABBotT Theoretical Neuroscience. (Cambridge, MIT Press, 2001).

[DM01] S. N. Dorogovtsev and J. F. F. Mendes Scaling properties of scale-free evolving networks: ?Continuous approach Phys. Rev. E 63 $(2001)(5)$.

[DNM06] L. Donetti, F. Neri and M. A. MuÑoz Optimal network topologies: expanders, cages, Ramanujan graphs, entangled networks and all that J. Stat. Mech: Theory Exp. 2006 (2006)(08) P08.007-P08.007.

$\left[\mathrm{DTD}^{+} 04\right]$ M. Denker, M. Timme, M. Diesmann, F. Wolf and T. Geisel Breaking synchrony by heterogeneity in complex networks Phys. Rev. Lett. 92 (2004)(7) 074.103.

[EK90] R. J. ElBle and W. C. KolleR Tremor (John Hopkins University Press, Baltimore, USA, 1990). 
[EPG95] U. ERnst, K. PAWELZIK and T. Geisel Synchronization induced by temporal delays in pulse-coupled oscillators Phys. Rev. Lett. 74 (1995) 1570.

[EPG98] U. ERnst, K. PawelzIK and T. Geisel Delay-induced multistable synchronization of biological oscillators Phys. Rev. E 57 (1998) 2150 .

[ER05] A. EdeLman and N. R. RAO Random matrix theory Acta Numerica 14 (2005) 233-297.

[Est10] E. Estrada Network Science (Springer-Verlag New York, 2010).

[Fag07] G. FAGiolo Clustering in complex directed networks Phys. Rev. E 76 (2007) 026.107.

[FDBV01] I. J. FArkas, I. Derényi, A.-L. BARAbÁsi and T. ViCsek Spectra of "real-world" graphs: Beyond the semicircle law Phys. Rev. E 64 (2001)(2) 12 .

$\left[\right.$ FJC $\left.^{+} 00\right] \quad$ K. Fink, G. Johnson, T. Carroll, D. Mar and L. Pecora Three coupled oscillators as a universal probe of synchronization stability in coupled oscillator arrays Phys. Rev. E 61 (2000)(5) 5080-5090.

[FJD06] J. Feng, V. JiRsa and M. Ding Synchronization in networks with random interactions: Theory and applications Chaos 16 (2006) 015.109.

[FM91] Y. Fyodorov and A. Mirlin Localization in ensemble of sparse random matrices Phys. Rev. Lett. 67 (1991)(15) 2049-2052.

[FPW06] G. Filatrella, N. F. Pedersen and K. Wiesenfeld Synchronization of Josephson vortices in multi-junction systems Physica C 437 (2006) 65-68.

[Fre77] L. Freeman A set of measures of centrality based upon betweenness Sociometry 40 (1977) 35-41.

[GD07] M.-O. Gewaltig and M. Diesmann NEST (NEural Simulation Tool) Scholarpedia 2 (2007)(4) 1430.

[GdBLC03] T. S. Gardner, D. Di Bernardo, D. Lorenz and J. J. Collins Inferring Genetic Networks and Identifying Compound Mode of Action via Expression Profiling Science 301 (2003)(5629) 102-105.

[GGT11] C. Grabow, S. Grosskinsky and M. Timme Speed of complex network synchronization Eur. Phys. J. B 84 (2011)(4) 613-626. 
[GGT12] C. Grabow, S. Grosskinsky and M. Timme Small-World Network Spectra in Mean-Field Theory Phys. Rev. Lett. 108 (2012)(21) 218.701.

[GHGT10] C. Grabow, S. M. Hill, S. Grosskinsky and M. Timme Do small worlds synchronize fastest? Europhys. Lett. 90 (2010)(4) 48.002 .

[Gil59] E. GilBerT Random Graphs Ann. Math. Stat. 30 (1959) $1141-1144$.

[Gir85] V. GiRko Eigenvalues of Asymmetric matrices Theory Probab. Appl. 29 (1985) 694.

[GK02] W. Gerstner and W. M. Kistler Spiking Neural Models. (Cambridge University Press, Cambridge, England, 2002).

[GM94] M. GelL-MAnN The Quark and the Jaguar (Freeman, New York, 1994).

[GR07] I. Gradshteyn and I. Ryzhik Table of Integrals, Series and Products (Academic Press, 2007).

[Gra01] R. GRAY Toeplitz and circulant matrices: A review http://ee.stanford.edu/gray/toeplitz.pdf (2001).

[GSKK06] K. Goh, G. Salvi, B. Kahng and D. Kim Skeleton and Fractal Scaling in Complex Networks Phys. Rev. Lett. 96 (2006).

[GT10] F. Götze and A. Tikhomirov The circular law for random matrices Ann. Probab. 38 (2010)(4) 1444-1491.

[GVL96] G. Golub and C. Van LoAn Matrix Computations (Johns Hopkins Studies in Math. Sci., 1996).

[HC99] R. Horn and J. C.R. Matrix analysis (Cambridge University Press, Cambridge, UK, 1999).

[HCAB05] D. Hwang, M. Chavez, A. Amann and S. Boccaletti Synchronization in complex networks with age ordering Phys. Rev. Lett. 94 (2005)(13) 138.701.

[HClP09] L. Huang, Q. Chen, Y. Lai and L. Pecora Generic behavior of master-stability functions in coupled nonlinear dynamical systems Phys. Rev. E 80 (2009)(3) 36.204.

[HCP94a] J. Heagy, T. Carroll and L. PeCora Experimental and numerical evidence for riddled basins in coupled chaotic systems Phys. Rev. Lett. 73 (1994)(26) 3528-3531. 
[HCP94b] J. Heagy, T. CARroll and L. PeCora Synchronous chaos in coupled oscillator systems Phys. Rev. E 50 (1994)(3) 1874-1885.

[HK03] T. Haveliwala and S. Kamvar The Second Eigenvalue of the Google Matrix Technical Report 2003-20 Stanford InfoLab (2003).

[HPC95] J. Heagy, L. Pecora and T. CARroll Short wavelength bifurcations and size instabilities in coupled oscillator systems Phys. Rev. Lett. 74 (1995)(21) 4185-4188.

[JJ01] J. Jost and M. Joy Spectral properties and synchronization in coupled map lattices Phys. Rev. E 65 (2001)(1).

[JMT08] S. Jahnke, R.-M. Memmesheimer and M. Timme Stable Irregular Dynamics in Complex Neural Networks Phys. Rev. Lett. 100 (2008)(4) 048.102.

[JSB00] S. Jespersen, I. M. Sokolov and A. Blumen Relaxation Properties of Small-World Networks J. Chem. Phys. 113 (2000)(7652).

[KER ${ }^{+}$09] W. Kinzel, A. Englert, G. Reents, M. Zigzag and I. Kanter Synchronization of networks of chaotic units with time-delayed couplings Phys. Rev. E 79 (2009)(5).

[KJJ96] E. KAnDEL, S. JH and T. JEsSELL Neurowissenschaften: Eine Einführung (Spektrum Akademischer Verlag, 1996).

[KKK02] I. KANTER, W. KINZEL and E. KANTER Secure exchange of information by synchronization of neural networks Europhys. Lett. 57 (2002)(1) 141-147.

[Kur84] Y. Kuramoto Chemical Oscillations, Waves and Turbulence (Springer, Berlin, 1984).

[KVM00] G. Kozyreff, A. Vladimirov and P. Mandel Global coupling with time delay in an array of semiconductor lasers Phys. Rev. Lett. 85 (2000)(18) 3809-3812.

[KvM11] R. KÜHN and J. VAn MOURIK Spectra of modular and small-world matrices J. Phys. A 44 (2011)(16) 165.205.

[LBH09] K. Lehnertz, S. Bialonski and M. Horstmann Synchronization phenomena in human epileptic brain networks J. Neurosci. Methods 183 (2009)(1).

[LKJ06] S. LEE, P. KIm and H. JEONG Statistical properties of sampled networks Phys. Rev. E 73 (2006) 016.102.

[LT85] P. LANCASTER and M. TismenETSKy The theory of matrices second edition with applications (Academic, Orlando, 1985). 
[MC01] D. MCCORMICK and D. Contreras On the cellular and network bases of epileptic seizures Annu. Rev. Physiol. 63 (2001)(1) $815-846$.

[McM02] D. MCMiLLEN Synchronizing genetic relaxation oscillators by intercell signaling Proc. Natl. Acad. Sci. U.S.A. 99 (2002)(2) 679-684.

[Meh91] M. Menta Random Matrices (Academic Press, New York, 1991).

[MF91] A. MirLin and Y. Fyodorov Universality of level correlation function of sparse random matrices J. Phys. A (1991).

[MHH98] K. MCCAnn, A. Hastings and G. Huxel Weak trophic interactions and the balance of nature Nature 395 (1998)(6704) 794-798.

[Mil10] J. G. Milton Epilepsy as a dynamic disease: A tutorial of the past with an eye to the future Epilepsy and Behavior 18 (2010)(1-2) $33-44$.

[MM08] P. N. MCGRAW and M. MenZINGER Laplacian spectra as a diagnostic tool for network structure and dynamics Phys. Rev. E 77 (2008)(3) 14 .

[MMZ04] S. C. Manrubia, A. S. Mikhailov and D. H. Zannette Emergence of dynamical order (World Scientific, 2004).

[MO04] F. Mori and T. OdAGAKI The Laplacian Spectra of Small-World Networks J. Phys. Soc. Jpn. 73 (2004)(12) 3294-3298.

[Mon99] R. Monasson Diffusion, localization and dispersion relations on "small-world" lattices Eur. Phys. J. B 12 (1999)(4) 555-567.

[MPBT04] Y. Maistrenko, O. Popovych, O. Burylko and P. Tass Mechanism of Desynchronization in the Finite-Dimensional Kuramoto Model Phys. Rev. Lett. 93 (2004) 084.102.

[MS90] R. Mirollo and S. Strogatz Synchronization of pulse-coupled biological oscillators Siam J. Appl. Math. 50 (1990)(6) 366.

[MS08] R. Morgan and I. Soltesz Nonrandom connectivity of the epileptic dentate gyrus predicts a major role for neuronal hubs in seizures Proc. Natl. Acad. Sci. U.S.A. 105 (2008)(16) 6179.

[MZK05a] A. Motter, C. Zhou and J. KurThs Enhancing complex-network synchronization Europhys. Lett. 69 (2005)(3) 334.

[MZK05b] A. MotTer, C. Zhou and J. KurThs Network synchronization, diffusion, and the paradox of heterogeneity Phys. Rev. E 71 (2005) 016.116 . 
$\left[\mathrm{NCA}^{+} 04\right]$ T. Netoff, R. Clewley, S. Arno, T. KeCK and J. White Epilepsy in small-world networks J. Neurosci. 24 (2004)(37) 8075-8083.

[New10] M. Newman Networks: An Introduction (Oxford University Press, Inc., New York, NY, USA, 2010).

[NMLH03] T. Nishikawa, A. Motter, Y. Lai and F. Hoppensteadt Heterogeneity in oscillator networks: Are smaller worlds easier to synchronize? Phys. Rev. Lett. 91 (2003)(1) 014.101.

[NMW00] M. Newman, C. Moore and D. Watts Mean-field solution of the small-world network model Phys. Rev. Lett. 84 (2000)(14) 3201-3204.

[NNS97] H. F. Nijhout, L. NAdel and D. L. Stein Pattern formation in the physical and biological sciences (Addison-Wesley Publishing Company, 1997).

[NW99] M. NEWMAN and D. WATTS Renormalization group analysis of the small-world network model Phys. Lett. A 263 (1999)(4-6) 341-346.

[OlPt10] S. Olmi, R. Livi, A. Politi and A. Torcini Collective oscillations in disordered neural networks Phys. Rev. E 81 (2010)(4).

[OS05] R. Olfati-Saber Ultrafast consensus in small-world networks Proc. Am. Control Conf. 4 (2005) 2371.

[Ott93] E. Oтт Chaos in Dynamical Systems (Cambridge University Press, New York, 1993).

[PC90] L. PECORA and T. CARroll SYNCHRONIZATION IN CHAOTIC SYSTEMS Phys. Rev. Lett. 64 (1990)(8) 821-824.

[PC98] L. PECORA and T. CARROLL Master stability functions for synchronized coupled systems Phys. Rev. Lett. 80 (1998)(10) 2109-2112.

[PCJ ${ }^{+}$97] L. Pecora, T. Carroll, G. Johnson, D. Mar and J. Heagy Fundamentals of synchronization in chaotic systems, concepts, and applications Chaos 7 (1997) 520.

[Pec98] L. PECORA Synchronization conditions and desynchronizing patterns in coupled limit-cycle and chaotic systems Phys. Rev. E 58 (1998)(1) 347-360.

[Per10] T. PEREIRA Hub synchronization in scale-free networks Phys. Rev. E (2010). 
[PHT05] O. Popovych, C. Hauptmann and P. Tass Effective Desynchronization by Nonlinear Delayed Feedback Phys. Rev. Lett. $94(2005)(16)$.

[PlR05] A. Pluchino, V. Latora and A. Rapisarda Changing opinions in a changing world:A new perspective in sociophysics Int. J. Mod. Phys. C 16 (2005) 515.

[Por65] C. PorTer (ed.) Statistical Theory of Spectra: Fluctuations (Academic Press, New York, 1965).

[PRK01] A. Pikovsky, M. Rosenblum and J. Kurths Synchronization, A universal concept in nonlinear sciences volume 12 of Cambridge Nonlinear Science Series (Cambridge University Press, Cambridge, UK, 2001).

[QHC $\left.{ }^{+} 08\right]$ G. X. Qi, H. B. Huang, L. Chen, H. J. Wang and C. K. Shen Fast synchronization in neuronal networks Europhys. Lett. 82 (2008)(3) 38.003.

$\left[\mathrm{QHS}^{+} 08\right]$ G. X. QI, H. B. Huang, C. K. Shen, H. J. Wang and L. Chen Predicting the synchronization time in coupled-map networks Phys. Rev. E 77 (2008)(5) 056.205.

[RAKK05] G. J. Rodgers, K. Austin, B. Kahng and D. Kim Eigenvalue spectra of complex networks J. Phys. A: Math. Gen. 38 (2005)(43) 9431-9437.

[RL11] A. RothKEGEL and K. LEHNERTz Recurrent events of synchrony in complex networks of pulse-coupled oscillators Europhys. Lett. 95 (2011)(3) 38.001.

[Rod88] G. Rodgers Density of states of a sparse random matrix Phys. Rev. B (1988).

[Roe76] O. E. Roessler An equation for continuous chaos Phys. Lett. A 57 (1976).

[SB09] O. SPORNS and E. Bullmore Complex brain networks: graph theoretical analysis of structural and functional systems Nat. Rev. Neurosci. 10 (2009)(3) 186-198.

[SBN09] J. Sun, E. Bollt and T. Nishikawa Master stability functions for coupled nearly identical dynamical systems Europhys. Lett.) $\mathbf{8 5}$ (2009) 60.011.

[SC02] G. SemerJian and L. F. Cugliandolo Sparse random matrices: the eigenvalue spectrum revisited J. Phys. A 35 (2002) 4837. 
[SCSS88] H. Sommers, A. Crisanti, H. Sompolinsky and Y. Stein Spectrum of large random asymmetric matrices Phys. Rev. Lett. 60 (1988) 1895.

[SDM08] A. N. Samukhin, S. N. Dorogovtsev and J. F. F. Mendes Laplacian spectra of, and random walks on, complex networks: Are scale-free architectures really important? Phys. Revi. E $\mathbf{7 7}$ (2008)(3) 19.

[SHK07] O. Sporns, C. Honey and R. KÖTter Identification and classification of hubs in brain networks Plos One 2 (2007)(10) e1049.

[Sip05] S. T. SiPILA Depolarizing GABA Acts on Intrinsically Bursting Pyramidal Neurons to Drive Giant Depolarizing Potentials in the Immature Hippocampus Journal of Neuroscience 25 (2005)(22) 5280-5289.

[SR94] M. Schlesinger and N. RAmankutTy An oscillation in the global climate system of period 65-70 years Nature 367 (1994)(6465) 723-726.

[SR07] C. STAM and J. REIJNEVELD Graph theoretical analysis of complex networks in the brain Nonlinear Biomedical Physics (2007).

[Str01] S. Strogatz Exploring complex networks Nature 410 (2001) 268-276.

[Str04] S. Strogatz Sync: The emerging science of spontaneous order (Penguin Books, London, UK, 2004).

[SvRE98] H. Schulz, U. vON RAD and H. ERlenkeuser Correlation between Arabian Sea and Greenland climate oscillations of the past 110,000 years Nature 393 (1998)(6680) 54-57.

[TFM96] S. Thorpe, D. Fize and C. Marlot Speed of processing in the human visual system Nature 381 (1996)(6582) 520-522.

[TGW06] M. Timme, T. Geisel and F. Wolf Speed of synchronization in complex networks of neural oscillators: Analytic results based on Random Matrix Theory Chaos 16 (2006)(1) 015.108.

[Tim06] M. Timme Does dynamics reflect topology in directed networks? Europhys. Lett. 76 (2006) 367.

[Tim07] M. Timme Revealing Network Connectivity from Response Dynamics Phys. Rev. Lett. 98 (2007)(22) 224.101.

[TM69] J. Travers and S. Milgram An experimental study of the small world problem Sociometry 32 (1969)(4) 425-443. 
[TMK10] R. TÖNJEs, N. MASUdA and H. KORI Synchronization transition of identical phase oscillators in a directed small-world network Chaos 20 (2010)(3) 033.108.

[TPM98] M. Tsodyks, K. PAWElzIK and H. MARKRAM Neural networks with dynamic synapses Neural Computation 10 (1998)(4) 821-835.

[Tuc88] H. C. TUCKWELL Introduction to theoretical neurobiology, volumes 1 and 2 (Cambridge Studies in Mathematical Biology. Cambridge University Press, Cambridge, England, 1988).

[TV09] T. TAO and V. Vu From the Littlewood-Offord Problem to the Circular Law: Universality of the Spectral Distribution of Random Matrices Bulletin AMS 46 (2009)(3) 377-396.

[TW08] M. Timme and F. WolF The simplest problem in the collective dynamics of neural networks: is synchrony stable? Nonlinearity $\mathbf{2 1}$ (2008)(7) 1579-1599.

[TWG02] M. Timme, F. Wolf and T. Geisel Coexistence of regular and irregular dynamics in complex networks of pulse-coupled oscillators Phys. Rev. Lett. 89 (2002)(25) 258.701.

[TWG03] M. Timme, F. Wolf and T. Geisel Unstable attractors induce perpetual synchronization and desynchronization Chaos 13 (2003) 377.

[TWG04] M. Timme, F. Wolf and T. Geisel Topological Speed Limits to Network Synchronization Phys. Rev. Lett. 92 (2004) 074.101.

[TYHC03] J. Tegnér, M. K. S. Yeung, J. Hasty and J. J. Collins Reverse engineering gene networks: Integrating genetic perturbations with dynamical modeling Proc. Natl. Acad. Sci. U.S.A. 100 (2003)(10) 5944-5949.

[UM03] N. UCHIDA and Z. MAINEN Speed and accuracy of olfactory discrimination in the rat Nature Neurosci. 6 (2003)(11) 1224-1229.

[Var05] R. S. VARGA Geršgorin and his circles (Springer series in computational mathematics vol. 36, Springer-Verlag, Berlin, Heidelberg, New York, 2005).

[VDHS $\left.{ }^{+} 03\right]$ M. Van Duijn, M. Huisman, F. Stokman, F. Wasseur and E. ZEGGELINK Clustering in complex directed networks J. Math. Sociol. 27 (2003).

[WF01] A. WAGNER and D. A. FelL The small world inside large metabolic networks Proc. R. Soc. London, Ser. B 268 (2001)(1478) 1803-1810. 
[Whi05] J. Whitfield Complex systems: Order out of chaos Nature 436 (2005)(7053) 905-907.

[Wie96] K. WiESENFELD New results on frequency-locking dynamics of disordered Josephson arrays Physica B 222 (1996)(4) 315-319.

[Wig51] E. P. Wigner On the statistical distribution of the widths and spacings of nuclear resonance levels Proc. Cambridge Philos. Soc. 47 (1951) 790.

[Wil96] R. J. WiLson Introduction to graph theory (Addison-Wesley, 1996).

[WM00] R. Williams and N. Martinez Simple rules yield complex food webs Nature 404 (2000)(6774) 180-183.

[WP08] I. WEDEKIND and U. PARLITZ Mode synchronization of external cavity semiconductor lasers Int. J. Bifurcation Chaos 18 (2008)(4) 1199-1209.

[WS98] D. WatTs and S. Strogatz Collective dynamics of 'small-world' networks Nature 393 (1998) 440-442.

[ZBH09] R. Zillmer, N. Brunel and D. Hansel Very long transients, irregular firing, and chaotic dynamics in networks of randomly connected inhibitory integrate-and-fire neurons Phys. Rev. E 79 (2009)(3) 031.909.

[Zie01] K. Ziemelis Nature Insight: Complex Systems (2001).

[ZK06] C. ZHOU and J. KuRTHS Hierarchical synchronization in complex networks with heterogeneous degrees Chaos 16 (2006)(1) 015.104.

[ZlPT06] R. Zillmer, R. Livi, A. Politi and A. Torcini Desynchronization in diluted neural networks Phys. Rev. E $\mathbf{7 4}$ (2006)(3).

[ZLPT07] R. Zillmer, R. Livi, A. Politi and A. Torcini Stability of the splay state in pulse-coupled networks Phys. Rev. E 76 (2007)(4) 046.102.

[ZLZK10] G. Zamora-López, C. Zhou and J. Kurths Cortical hubs form a module for multisensory integration on top of the hierarchy of cortical networks Front. in Neuroinf. 4 (2010).

[ZTGW04] A. Zumdieck, M. Timme, T. Geisel and F. Wolf Long Chaotic Transients in Complex Networks Phys. Rev. Lett. 93 (2004)(24) 244.103 . 


\section{Nomenclature}

Table 6.1: List of abbreviations. Additional abbreviations used in Chapter 5 are listed in Tables 5.4.2 and 5.4.2 on page 84 .

\begin{tabular}{llrr}
\hline Abbreviation & Description & Reference & Page \\
\hline cLIF & conductance-based leaky integrate-and-fire & 5.4 & 84 \\
CSPO & close-to-synchronous periodic orbit & 5.3 & 81 \\
EPSP & excitatory postsynaptic potential & 5 & 78 \\
exc & excitatory & 5.4 & 84 \\
GABA & $\gamma$-amino-butyric-acid & 5 & 77 \\
GDP & giant depolarizing potential & 5 & 77 \\
HC & high-connectivity & 5 & 77 \\
IN & interneuron & 5 & 77 \\
inh & inhibitory & 5.4 & 84 \\
K & Kuramoto oscillators & 3.1 & 29 \\
LC & low-connectivity & 5 & 77 \\
LIF & leaky integrate-and-fire & 3.4 .3 & 37 \\
MSF & master stability function & 3.4 .2 & 32 \\
NEST & Neural Simulation Technology & 5.4 & 83 \\
PC & pulse-coupled oscillators & 3.1 & 29 \\
PSC & post-synaptic current & 5.4 & 86 \\
pyr & pyramidal neurons & 5.4 & 84 \\
R & Rössler oscillators & 3.1 & 29 \\
SCC & strongly connected component & 2.1 .3 & 17 \\
SW & small world & 2.2 .1 & 20 \\
syn & synaptic & 5.4 & 84 \\
\hline & & &
\end{tabular}


Table 6.2: List of symbols. Additional symbols used in Chapter 5 are listed in Tables 5.4 .2 and 5.4 .2 on page 84 .

\begin{tabular}{|c|c|c|c|}
\hline Symbol & Description & Reference & Page \\
\hline$A$ & adjacency matrix & 2.1.1, eq. (2.1.1) & 15 \\
\hline$B$ & betweenness centrality & 2.1.3, eq. (2.1.11) & 18 \\
\hline$C$ & clustering coefficient & 2.1.3, eq. (2.1.18) & 19 \\
\hline$d_{\mathrm{K}}$ & maximal distance (Kuramoto) & 3.4.1, eq. (3.4.4) & 31 \\
\hline$d_{\mathrm{PC}}$ & maximal distance (pulse-coupled) & 3.4.3, eq. (3.4.23) & 36 \\
\hline$d_{\mathrm{R}}$ & maximal distance (Rössler) & 3.4 .2 , eq. (3.4.7) & 32 \\
\hline$D \ldots$ & Jacobian & 3.4 .2 & 32 \\
\hline$\delta_{\mathrm{K}}$ & perturbation (Kuramoto) & 3.4.1, eq. (3.4.2) & 31 \\
\hline$\delta_{\mathrm{PC}}$ & perturbation (pulse-coupled) & 3.4 .3 , eq. (3.4.21) & 36 \\
\hline$\delta_{\mathrm{R}}$ & perturbation (Rössler) & 3.4 .2 , eq. (3.4.8) & 32 \\
\hline$\Delta$ & delay & 2.2 .2 , eq. (2.2.6) & 23 \\
\hline$f$ & total weight & 4.2 , eq. (4.2.3) & 56 \\
\hline $\boldsymbol{F}$ & free dynamics function & 3.1, eq. (3.1.1) & 26 \\
\hline$\underset{\sim}{G}$ & stability matrix & 3.4 .3 , eq. (3.4.35) & 37 \\
\hline$\tilde{G}$ & nonlinear stroboscopic map & 3.4 .3 & 36 \\
\hline$\gamma$ & dissipation & 2.2 .2 , eq. (2.2.6) & 23 \\
\hline$h$ & Lyapunov exponent & 3.4 .2 & 34 \\
\hline$h_{1, \gamma}$ & master stability function & 3.4.2, eq. (3.4.14) & 34 \\
\hline $\boldsymbol{H}$ & interaction function & 3.1 , eq. (3.1.5) & 27 \\
\hline$I$ & external current & 2.2 .2 , eq. (2.2.6) & 23 \\
\hline$J$ & coupling matrix & 2.2.2, eq. (2.2.1) & 22 \\
\hline$k$ & degree & 2.1.3, eq. (2.1.4) & 16 \\
\hline$k^{\mathrm{bi}}$ & number of bilateral edges & 2.1 .3 , eq. (2.1.14) & 18 \\
\hline$k^{\text {in }} / k^{\text {out }}$ & in-/out-degree & 2.1.3, eq. (2.1.2) & 15 \\
\hline$k^{\text {tot }}$ & total degree & 2.1.3, eq. (2.1.14) & 18 \\
\hline$l_{i j}$ & length of a shortest path & 2.1 .3 , eq. (2.1.7) & 16 \\
\hline$L$ & average shortest path length & 2.1.3, eq. (2.1.8) & 16 \\
\hline$\Lambda$ & (scaled) graph Laplacian & 2.1.4, eqs. (2.1.22), (2.1.21) & 20 \\
\hline$\tilde{\Lambda}^{\mathrm{mf}}\left(\Lambda^{\mathrm{mf}}\right)$ & (scaled) mean field graph Laplacian & 4.2, eq. (4.2.9) & 58 \\
\hline$M$ & number of edges & 2.1 .3 , eqs. (2.1.3), (2.1.5) & $15 / 16$ \\
\hline$N$ & graph (network) size & 2.1 .1 & 15 \\
\hline$P$ & link probability in Erdös-Rényi graphs & 4.5 & 68 \\
\hline$p$ & rewiring probability & 3.6 & 50 \\
\hline$\phi_{i}$ & phases (pulse-coupled) & 2.2 .2 , eq.(2.2.6) & 23 \\
\hline$q$ & topological randomness & 2.2 .1 & 21 \\
\hline$\rho$ & density of states & 4.6, eq. (4.6.1) & 73 \\
\hline
\end{tabular}


Table 6.3: List of symbols. Additional symbols used in Chapter 5 are listed in Tables 5.4 .2 and 5.4 .2 on page 84 .

\begin{tabular}{|c|c|c|c|}
\hline Symbol & Description & Reference & Page \\
\hline$\sigma$ & global coupling constant & 2.2 .2 & 21 \\
\hline$\tau_{\mathrm{K}}$ & synchronization time (Kuramoto) & 3.4.1, eq. (3.4.5) & 31 \\
\hline$\tau_{\mathrm{PC}}$ & synchronization time (pulse-coupled) & 3.4 .3 , eq. (3.4.53) & 39 \\
\hline$\tau_{\mathrm{R}}$ & synchronization time (Rössler) & 3.4 .2 , eq. (3.4.16) & 35 \\
\hline$\Theta_{i}$ & phases (Kuramoto) & 2.2 .2 & 22 \\
\hline$T$ & period (pulse-coupled) & 3.4.3, eqs. (3.4.18), (3.4.27) & $35 / 36$ \\
\hline$U$ & rise function & 2.2.2, 3.4.3, eq. (3.4.29) & $23 ; 37$ \\
\hline$V_{i}$ & membrane potential & 2.2 .2 , eq. (2.2.6 & 23 \\
\hline$w_{1}, w_{2}$ & weights for individual edges & 4.2 , eqs. (4.2.6), (4.2.7) & 58 \\
\hline$\omega_{i}$ & oscillator frequencies (Kuramoto) & 2.2 .2 , eq. (2.2.2) & 22 \\
\hline
\end{tabular}

